\title{
Dimethyl Acetylenedicarboxylate: A Versatile Tool in Organic Synthesis
}

\author{
Constantinos G. Neochoritis, Tryfon Zarganes-Tzitzikas, Julia Stephanidou-Stephanatou*
}

Department of Chemistry, Laboratory of Organic Chemistry, Aristotle University of Thessaloniki, Thessaloniki 54124, Macedonia, Greece Fax+30(2310)997679; E-mail: ioulia@chem.auth.gr

Received: 03.10.2013; Accepted after revision: 05.11.2013

\begin{abstract}
This review presents the recent progress in the chemistry of dimethyl acetylenedicarboxylate (DMAD). The interest in and applications of this powerful reagent with more than 135 years of history have greatly increased in the last 10 years, further proving its versatility. Undoubtedly, DMAD can be a multi-tool in the quest of molecular complexity and diversity. The extreme structural diversity of the products described in this review illustrates the powerful potential of DMAD as a building block in organic synthesis.

1 Introduction

2 Michael Reactions

2.1 Sulfur as Nucleophile

2.2 Nitrogen as Nucleophile

2.3 Oxygen as Nucleophile

2.4 Addition to Carbon-Carbon Double Bonds

3 Cycloaddition Reactions

3.1 Diels-Alder Reactions ([4+2] Cycloadditions)

3.2 1,3-Dipolar Reactions ([3+2] Cycloadditions)

$3.3[2+2]$ Cycloadditions

$3.4[8+2]$ Cycloadditions

4 DMAD and the Generation of Zwitterions; Multicomponent Reactions (MCRs)

4.1 Phosphines and Derivatives

4.2 Amines

4.3 Isocyanides

4.4 Carbenes

4.5 Miscellaneous Reactions

5 Conclusion
\end{abstract}

Key words: DMAD, dimethyl acetylenedicarboxylate, Michael reactions, cycloadditions, multicomponent reactions

\section{$1 \quad$ Introduction}

Dimethyl acetylenedicarboxylate, commonly abbreviated as DMAD, is an electro-deficient alkyne diester. This ester, which exists as a liquid (density: $1,156 \mathrm{~g} / \mathrm{mL}, 25^{\circ} \mathrm{C}$ ) at room temperature (boiling point: $95-98^{\circ} \mathrm{C}$ ), is highly electrophilic. As such, DMAD is used as a dienophile and a dipolarophile in cycloaddition reactions. Today, it is being used increasingly in chemical synthesis as it has proven useful in carbon-carbon bond formations. DMAD is an extremely versatile tool for organic chemists and com-

SYNTHESIS 2014, 46, 0537-0585

Advanced online publication: 07.02.2014

DOI: 10.1055/s-0033-1340615; Art ID: SS-2013-E0659-R

(C) Georg Thieme Verlag Stuttgart $\cdot$ New York pletely new avenues have been explored for its use in combinatorial and multicomponent chemistry and heterocyclic synthesis. ${ }^{1}$ Following the pioneering discovery by Diels and Alder, ${ }^{2,3}$ the reactions of dimethyl acetylenedicarboxylate with heterocyclic compounds have been the subject of a great number of publications $\mathrm{s}^{4,5}$

This treatise assembles and presents the fundamental characteristics of DMAD chemistry, as well as current developments thereof. The focus is placed on Michael reactions, cycloadditions (Diels-Alder, 1,3-dipolar and [2+2] cycloadditions), and, of course, multicomponent reactions by generation of zwitterions (Scheme 1). Recent literature examples that are both illustrative of the power of this reagent in the construction of complex heterocyclic molecules and pivotal for the design of synthetic strategies toward natural or designed targets are discussed herein. This review seeks to highlight the 'power of DMAD' by examining selected examples of its elegant applications.

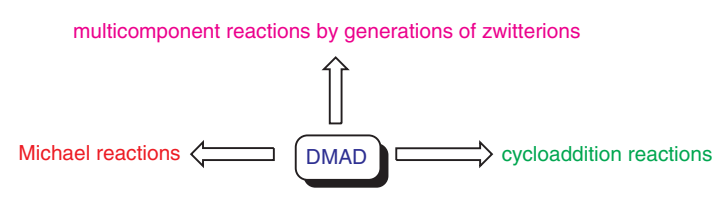

Scheme 1

DMAD is inexpensive and widely available, or it can be prepared from maleic acid (1) via a bromination-dehydrohalogenation sequence to furnish acetylene dicarboxylic acid (4), which upon esterification with methanol using sulfuric acid gives the desired dimethyl acetylenedicarboxylate (5). It is noteworthy that DMAD is still synthesized in exactly the same way as it was originally obtained (Scheme 2). ${ }^{6}$

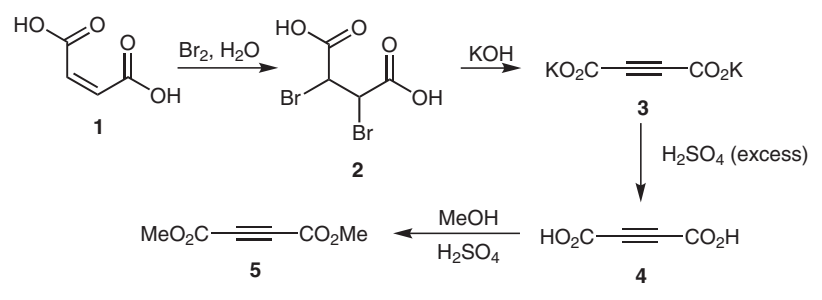

Scheme 2

\section{$2 \quad$ Michael Reactions}

DMAD is a powerful Michael acceptor and can take up various nucleophiles, most commonly sulfur and nitrogen 
(Scheme 3). A common strategy is to use it in an initial reaction as a Michael acceptor and then carry out a cyclization, usually through loss of $-\mathrm{OMe}$ or $-\mathrm{CO}_{2} \mathrm{Me}$. The majority of reactions have been carried out on heteroaromatic systems, such as pyridines, quinolines, isoquinolines, thiazoles, imidazoles, phenanthridines, quinoxalines, pyridazines and their substituted derivatives, with DMAD being used as a cyclizing agent.

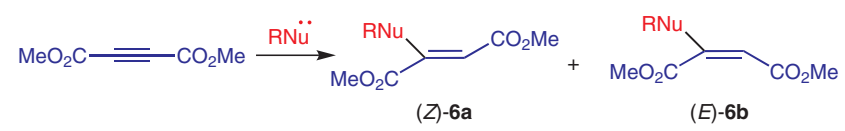

Scheme 3

\subsection{Sulfur as Nucleophile}

\subsubsection{Reactions with Thiourea, Thioamide and Thiosemicarbazide Derivatives}

Thiazole, thiazolidines, thiazolidinones and thiazinones are very important groups of heterocyclic compounds covering a broad spectrum of biological activity. The reaction between thioamides, thiosemicarbazides and thiourea derivatives 7 with DMAD is known to be a convenient and effective method for the preparation of the aforementioned heterocycles depending on the reaction conditions. Generally, the sulfur attacks the triple bond, resulting in the formation of thiolactam intermediate $\mathbf{8}$, followed by the aminolysis of an ester group (Scheme $4),{ }^{7,8}$ thus leading to the formation of either 1,3-thiazolidin-4-ones 9 or 1,3-thiazin-4-ones $\mathbf{1 0}$.

Ahmadi et al. ${ }^{9}$ developed a general strategy based on the reaction of thiourea derivatives 11 with DMAD (Scheme 5) to prepare 4-thiazolidinone derivatives 12. Furthermore, various thiosemicarbazone derivatives $\mathbf{1 3}$ (furfural, benzophenone, butanal, $p$-methoxybenzaldehyde, etc.) and DMAD reacted in ethyl acetate to give compounds 14 as the only products, although from the reaction in anhydrous methanol only compounds $\mathbf{1 5}$ were formed. It is noteworthy that the adducts $\mathbf{1 4}$ could be converted into products $\mathbf{1 5}$ by refluxing the reaction mixture in anhydrous methanol (Scheme 6). ${ }^{10}$

\section{Biographical Sketches}
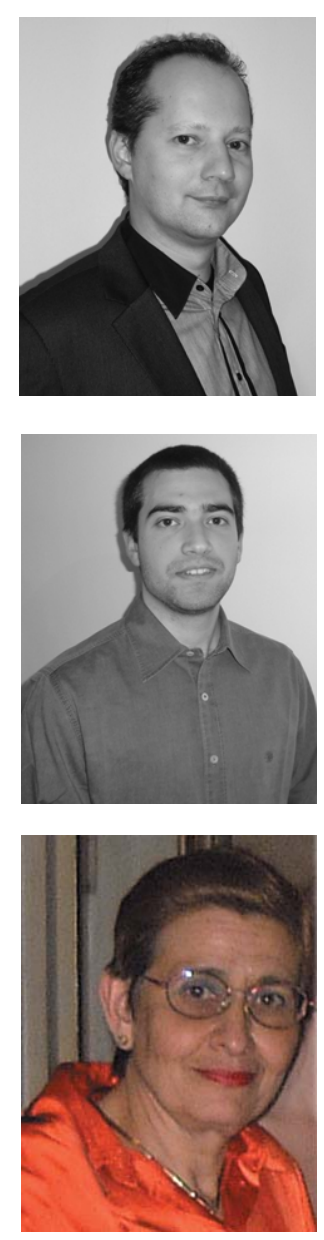

Constantinos G. Neochoritis was born in Thessaloniki, Macedonia, Greece in 1982. He received his MSc (2006) and his PhD (2011) in organic chemistry under

Tryfon Zarganes-Tzitzikas was born in Thessaloniki, Greece in 1988. He obtained his BSc degree in chemistry from the Aristotle interests include bioactive heterocycles, drug design and multicomponent reactions. He has produced more than 12 peer-reviewed papers.

Professors J. StephanidouStephanatou and C. Tsoleridis at Aristotle University of Thessaloniki.

bilitation in 1982, she rose through the ranks to Professor of Organic Chemistry (1992). She currently works on multicomponent reactions and heterocyclic chemistry. 


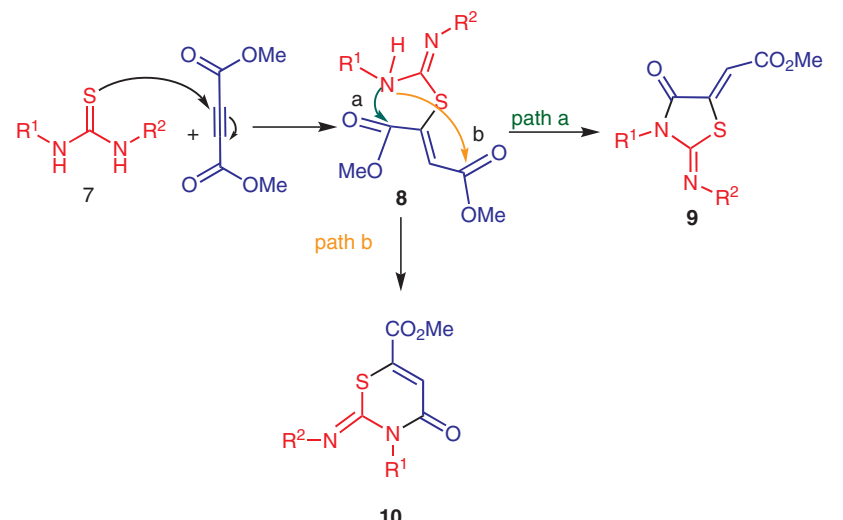

Scheme 4

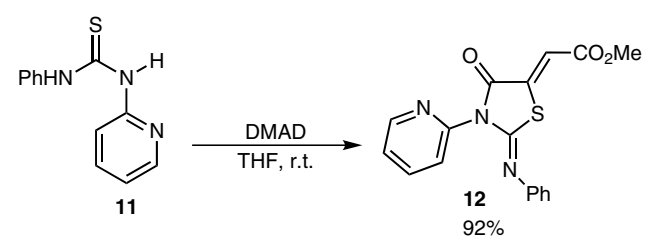

Scheme 5

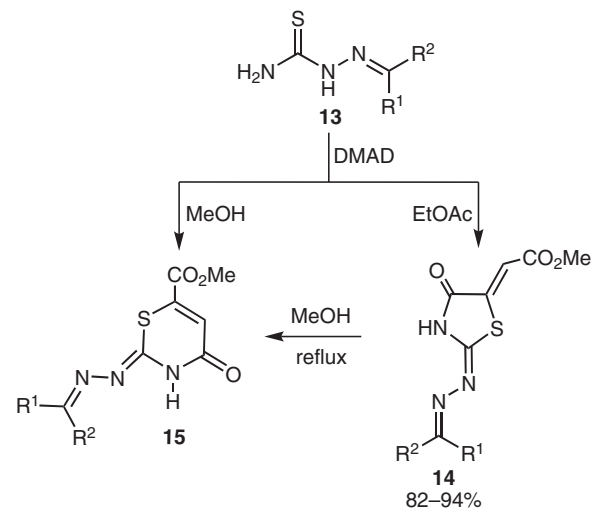

Scheme 6

In a more characteristic example, compounds 16, upon reaction with DMAD, yielded the 1,3-thiazol-4-ones $\mathbf{1 7}$ as the major products (Scheme 7) rather than the 1,3-thiazin4-ones $18 .{ }^{8}$ Likewise, an acridine reactant with a bulky substituent on $\mathrm{N}-1$, such as $\mathbf{1 9}$, produced the thiazolidinone 21 upon cyclization with DMAD (Scheme 8). ${ }^{11}$

Moreover, reactions of DMAD with the $N$-imidoylthioureas $\mathbf{2 2}$ in acetic acid afforded the thiadiazepines $\mathbf{2 3}$ in $65-84 \%$ yield (Scheme 9). ${ }^{12}$

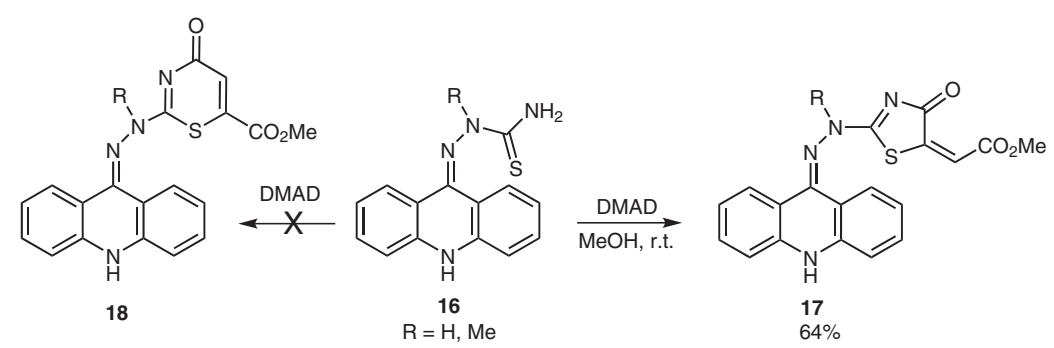

Scheme 7<smiles>CC(C)(C)NNC(=S)Nc1c2ccccc2nc2ccccc12</smiles>

20<smiles>CC(C)(C)NNC(=S)N=c1c2ccccc2[nH]c2ccccc12</smiles>

19<smiles>CCCCNN1C(=O)/C(=C/C(C)C)S/C1=N\c1c2ccccc2nc2ccccc12</smiles>

21

Scheme 8 
<smiles>COC(=O)C1=C(C(=O)OC)N(I)C(c2ccccc2)=NC(=N)S1</smiles>

\section{Scheme 9}

In recent decades, ferrocenes $(\mathrm{Fc})$ have attracted attention because of their wide utility in materials science and catalysis. ${ }^{13}$ Therefore, the DMAD-mediated cyclization of 1,5-bis(ferrocenylmethylene)thiocarbono hydrazide (24) and its $S$-methyl derivative $\mathbf{2 5}$ were studied, leading to the preparation of biologically promising sulfur heterocycles 26 and 27, and the methylthio-substituted nitrogen heterocycles 28 and 29, respectively (Scheme 10). ${ }^{14}$
In addition, by treatment of the ferrocenyl thiosemicarbazone derivatives 30 with DMAD in refluxing acetonitrile, three types of cyclic products $(\mathbf{3 1}, \mathbf{3 2}$ and 33) were isolated in moderate to good yields. The methylthio derivatives $\mathbf{3 4}$, obtained by selective alkylation of 30, were also allowed to react with DMAD under the same conditions, thereby affording complex mixtures of methylthio-substituted products including pyrimidones 35 , imidazolones 36 and fumarates 37 (Scheme 11). ${ }^{15}$

Reaction of DMAD with dithizone (2:1 molar ratio) in methanol resulted in the formation of thiadiazine 39 through a Michael-type addition and a Diels-Alder [4+2]cycloaddition reaction. In contrast, the reaction of DMAD with dithizone (1:1 molar ratio) in methanol gave compound 40 (Scheme 12). ${ }^{16}$ Thiadiazine derivatives are widely used as nematicides, fungicides, herbicides and insecticides. In addition, some thiadiazine derivatives show activity against tripanosoma cruzi amastigotes. ${ }^{17}$

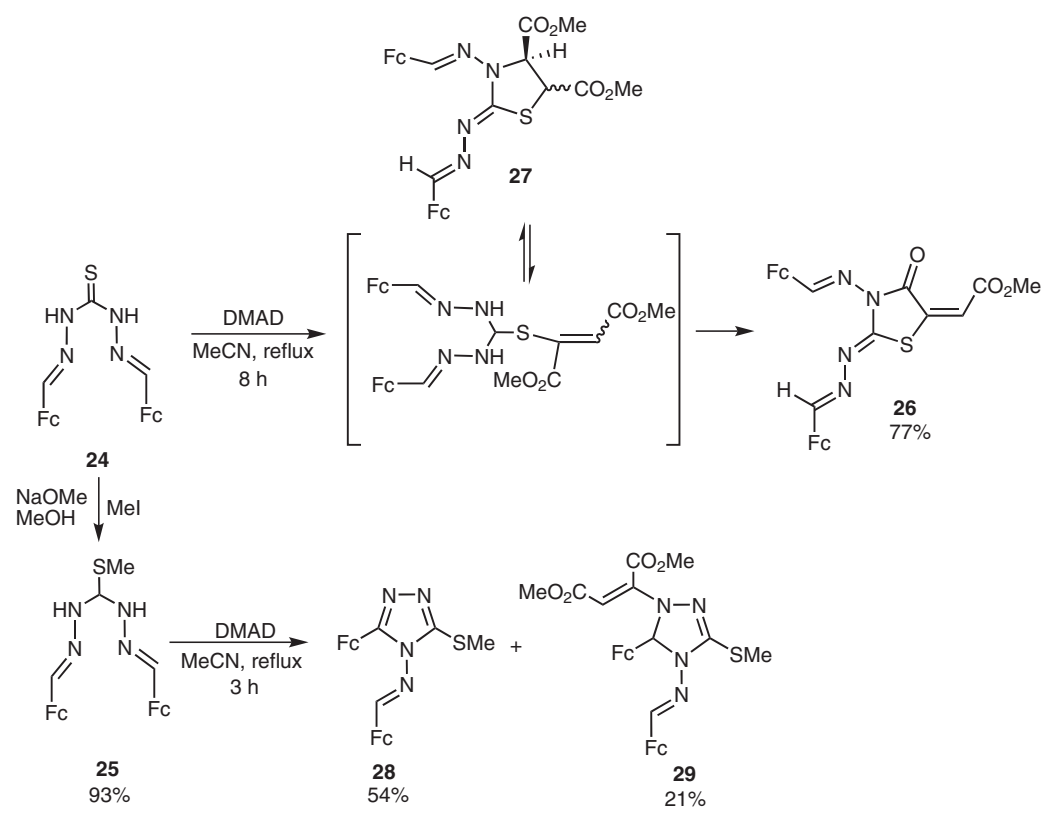

Scheme 10

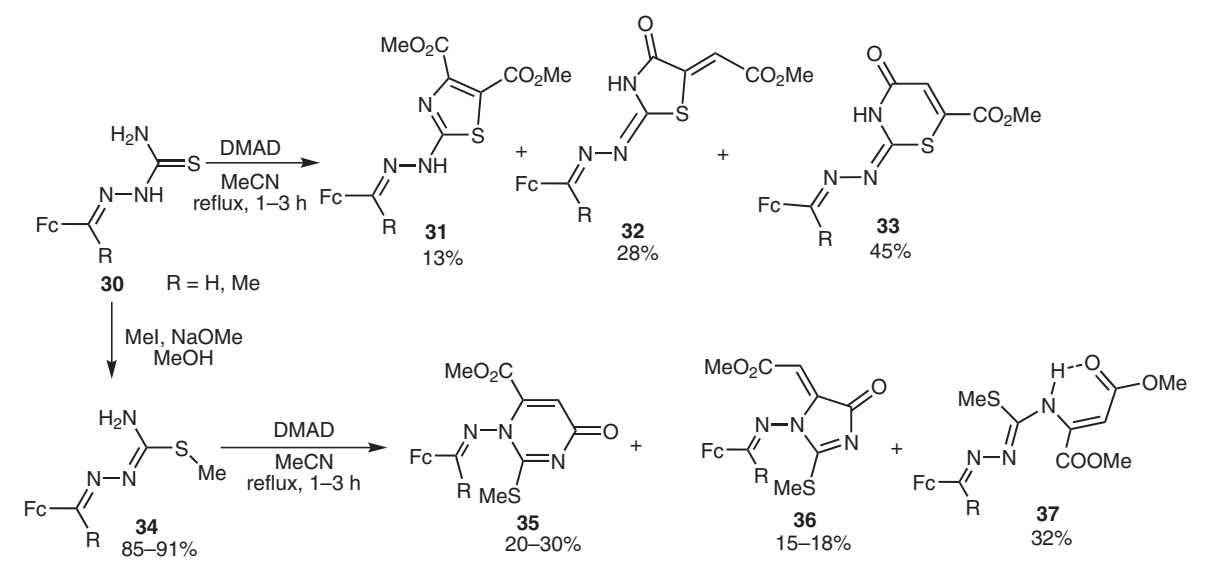

Scheme 11 


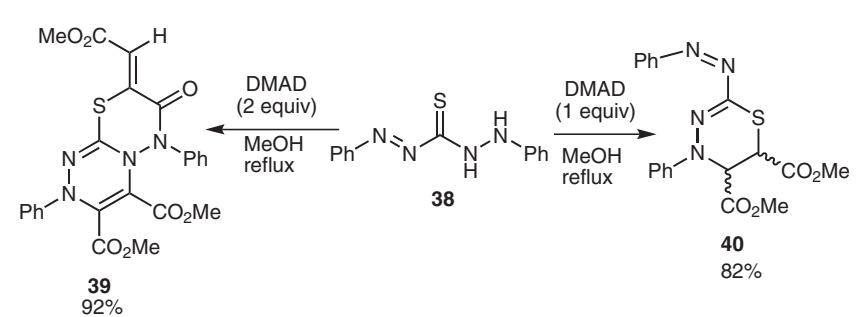

Scheme 12

\subsubsection{Reaction with Thiones and Thiols}

An example of the 'DMAD strategy' for the synthesis of heterocycles was described by Neochoritis et al. ${ }^{18}$ It is well known that Michael-type amine and thione addition to acetylenic esters leads to $E / Z$ isomeric mixtures. The reaction of DMAD with 1-arylaminoimidazole-2-thiones $\mathbf{4 1}$ in the presence of a base through a Michael reaction af- forded imidazolothiazoles 43 . However, in the reaction of 41 with DMAD in the absence of a base, only the S-substituted products $\mathbf{4 2}$ were formed as an $E / Z$ mixture. A thorough investigation of this thio-Michael-type addition of DMAD and an explanation of the reaction's stereospecifity were reported (Scheme 13).

A systematic study of the reactions of DMAD with 5-mercaptoazoles 44, 47, 49 and pyridine-2-thiones 52 was also carried out and, as a result, a number of novel imidazothiazinones 45 , imidazothiazolones 46 , triazoles 48 , pyrazolothiazinones 51 and thiazolopyridines 53 were obtained. The size of the ring, formed in the reactions of cyclic thioamides with DMAD, was found to be dependent on the size of the starting heterocycle. Thus, a fivemembered thiazolidine ring condenses onto the pyridine ring whereas a six-membered thiazine ring is fused onto a five-membered azole ring (Scheme 14). ${ }^{19}$

\section{Scheme 13}

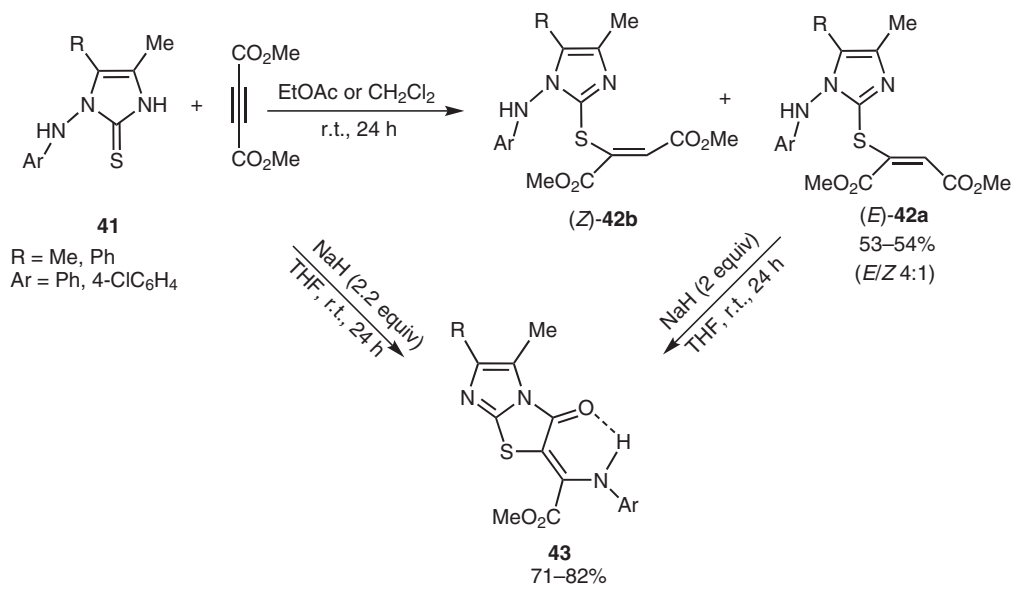

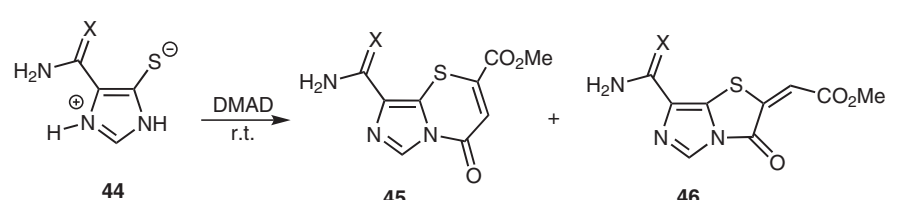

$x=0, S$

45

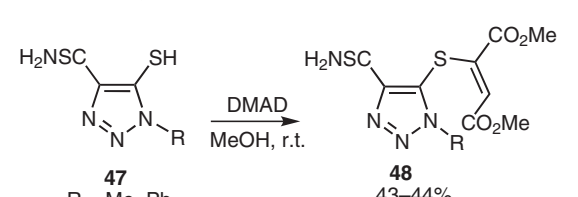

$\mathrm{R}=\stackrel{47}{\mathrm{Me}}, \mathrm{Ph}$

$\stackrel{48}{43-44 \%}$

$48-66 \%(45 / 46 \quad 9: 1)$
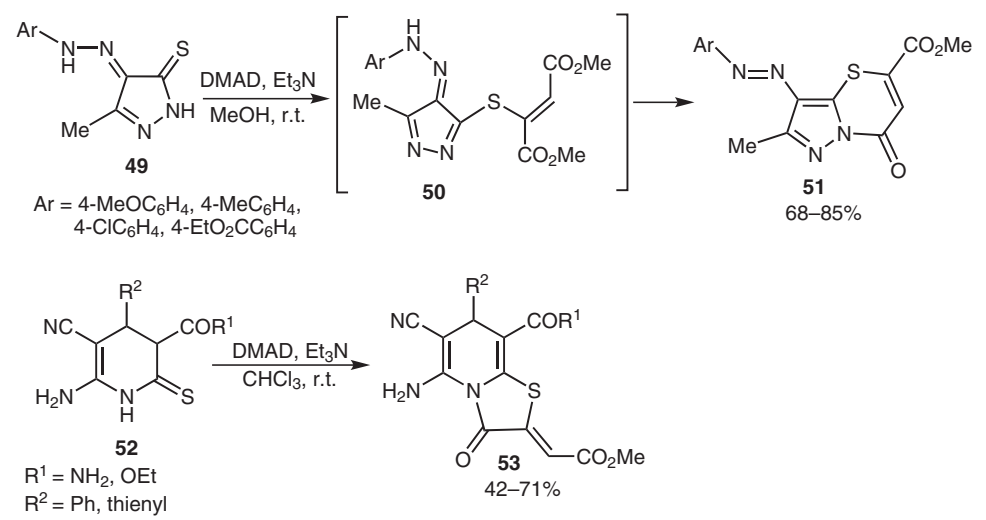

\section{Scheme 14}

(c) Georg Thieme Verlag Stuttgart $\cdot$ New York 
Furthermore, the reaction of quinazoline derivatives $\mathbf{5 4}$ with DMAD affording compounds $\mathbf{5 6}$ was investigated within a study of the varying reactivity of nitrogen and sulfur as nucleophiles with an electron-deficient ester (Scheme 15). ${ }^{9}$

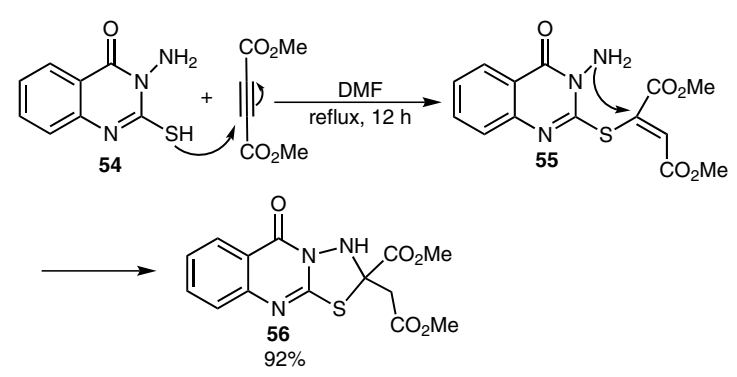

Scheme 15

The reaction of DMAD with thiols $\mathbf{5 7}$ in water was reported to lead to $E / Z$ isomeric mixtures of $\mathbf{5 8}$ in quantitative yield (Scheme 16). ${ }^{20}$

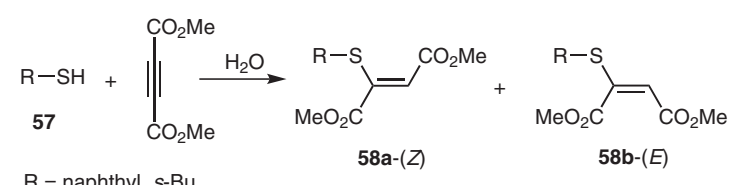

Scheme 16

In 2008, DMAD was used in the synthesis of 3 -substituted thieno[3,2-b] furan derivatives 61. The Michael reaction between methyl thioglycolate and DMAD, followed by in situ intramolecular cyclization, afforded the 3-hydroxythiophene 60 in $85 \%$ yield (Scheme 17). ${ }^{21}$

The reaction of DMAD with arylidenemalononitriles $\mathbf{6 2}$, in the presence of potassium isothiocyanate in acetonitrile, led to a mixture of cyanothiophene $\mathbf{6 3}$ and dicyanocyclopenta-1,3-diene derivatives 64 . It was proposed that

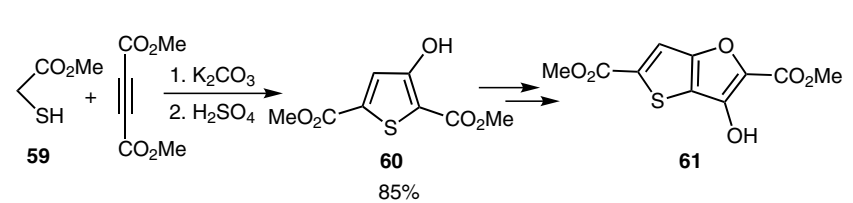

Scheme 17

this multicomponent reaction could have been started with the addition of isothiocyanate ion to DMAD (Michael reaction) followed by addition of $\mathbf{6 2}$ (Scheme 18). ${ }^{22}$

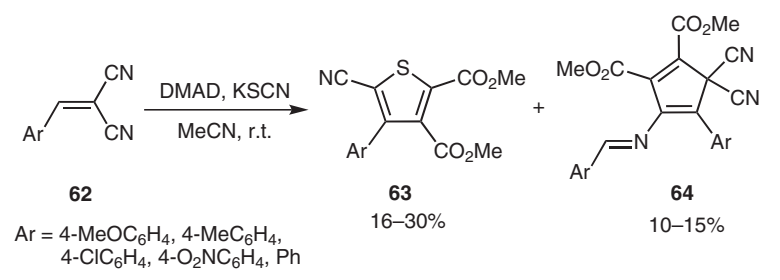

Scheme 18

\subsection{Nitrogen as Nucleophile}

\subsubsection{Reactions with Tertiary Amines and Hetero- cycles}

In recent years, there has been considerable interest in investigations of the reactivity of nitrogen-containing heterocycles with DMAD affording a wide range of products. For example, benzothiazines and benzothiazepines 65 and 68 were allowed to react with DMAD in methanol, affording the corresponding pyrrolo- and oxazolo- benzothiazines and benzothiazepines 67 and 70. It was suggested that the products were formed by the addition of one molecule of DMAD to one molecule of benzothiazine or benzothiazepine, with elimination of one molecule of methanol (Scheme 19). ${ }^{5}$

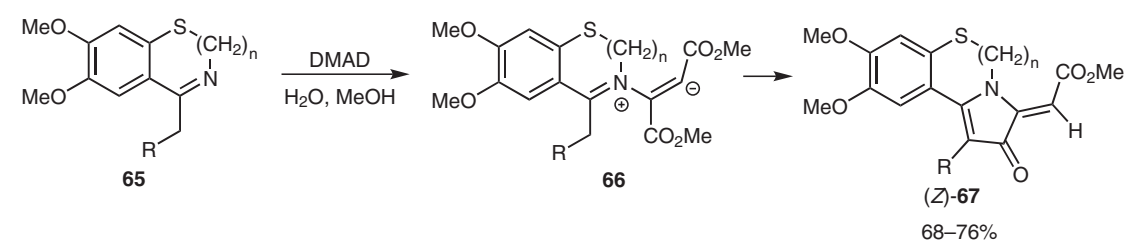

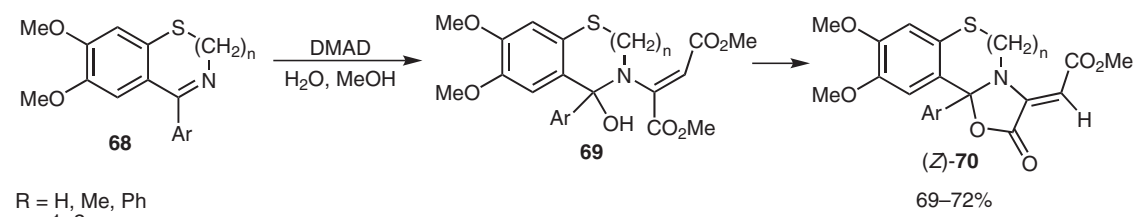

Scheme 19 
Fodor et al. ${ }^{23}$ reported that benzothiazines $\mathbf{7 1}$ reacted with DMAD in acetonitrile at room temperature, affording the regioisomeric ring systems 1,5 -thiazocines $\mathbf{7 3}$ and $\mathbf{7 5}$ in a ratio of approximately $1: 1$. DMAD was found to attack either the nitrogen or sulfur atom of 71. The key intermediates in the formation of 5,6-dihydro- $2 H$-1,5-benzothiazocines $\mathbf{7 3}$ were most probably the zwitterions $\mathbf{7 2}$, whereas the unexpected formation of 5,6-dihydro- $4 \mathrm{H}-1,5-$ benzothiazocines $\mathbf{7 5}$ was explained by invoking intermediates 74 (Scheme 20).

The reaction of pyrrolopyrimidine 76 with DMAD afforded, depending on the reaction conditions, the trifluoroacetylpyrroles $\mathbf{7 9}$ and $\mathbf{8 0}$ through the zwitterionic intermediates 77 and 78 (Scheme 21). ${ }^{24}$

Tetrahydropyrrolopyridines $\mathbf{8 1}$ and tetrahydropyridoindoles 86 underwent piperidine ring opening under the action of DMAD in acetonitrile, alcohols or aqueous dioxane, providing substituted pyrroles $\mathbf{8 2}-\mathbf{8 5}$ and $\mathbf{8 7}$, respectively (Scheme 22). ${ }^{25,26}$

In 2008, reactions of quinoline derivatives with DMAD were reported. The reaction began with a Michael addition of the tertiary amine in benzonaphthyridines $\mathbf{8 8}$ to the triple bond of the alkyne. The intermediate zwitterion $\mathbf{8 9}$ was further transformed by two pathways, A and B. Pathway A was conditioned by the acidic character of the $\mathrm{CH}_{2}$ group and led to ylide $\mathbf{9 0}$, which, by Stevens rearrangement (characteristic of ylides), was transformed into acryloyl-substituted naphthyridines $\mathbf{9 2}$. The implementation of pathway B was caused by the nucleophilic attack of the zwitterion on the nitrile group. The intermediate 91 was converted into succinate 93. Naphthyridines $94\left(\mathrm{R}^{1}=\mathrm{Bn}\right.$, $\mathrm{R}^{2}=\mathrm{H}$ and $\mathrm{R}^{1}={ }^{i} \mathrm{Pr}, \mathrm{R}^{2}=\mathrm{Br}$ ) did not react with DMAD in methanol at room temperature, whereas reflux led to the elimination of benzyl or isopropyl substituents and the formation of $\mathrm{N}$-substituted derivatives 95 (Scheme 23) ${ }^{27}$

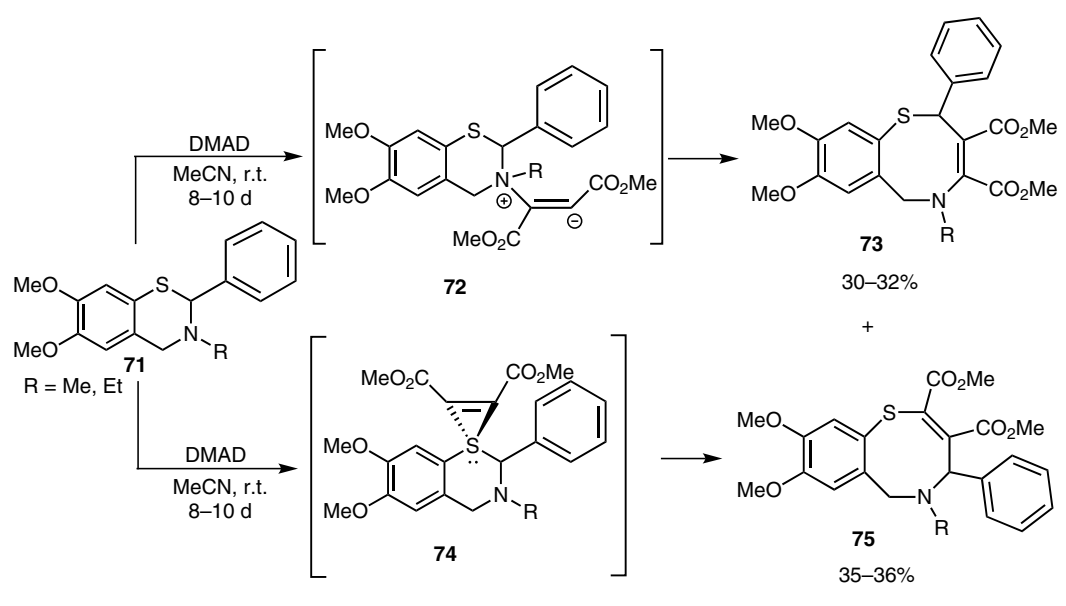

Scheme 20

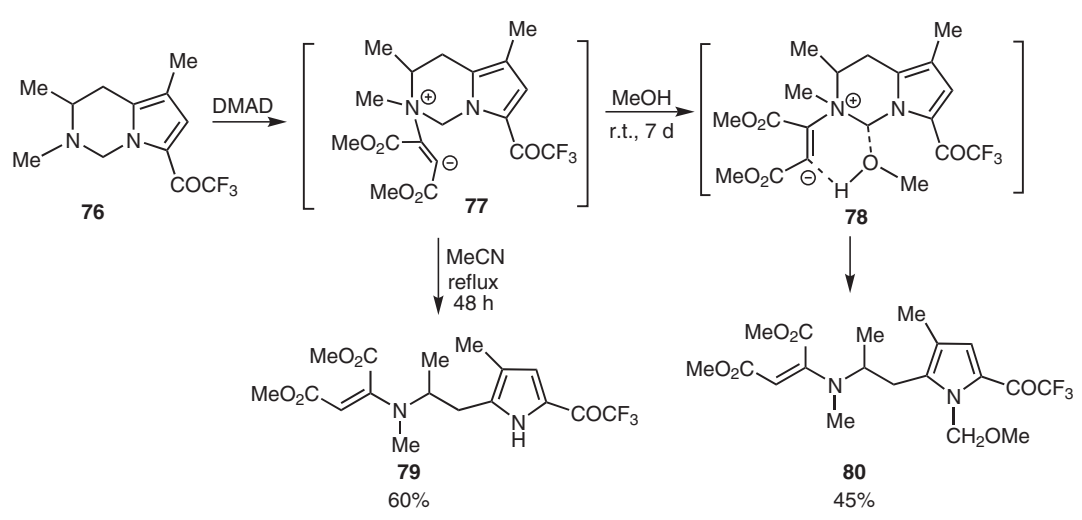

Scheme 21 


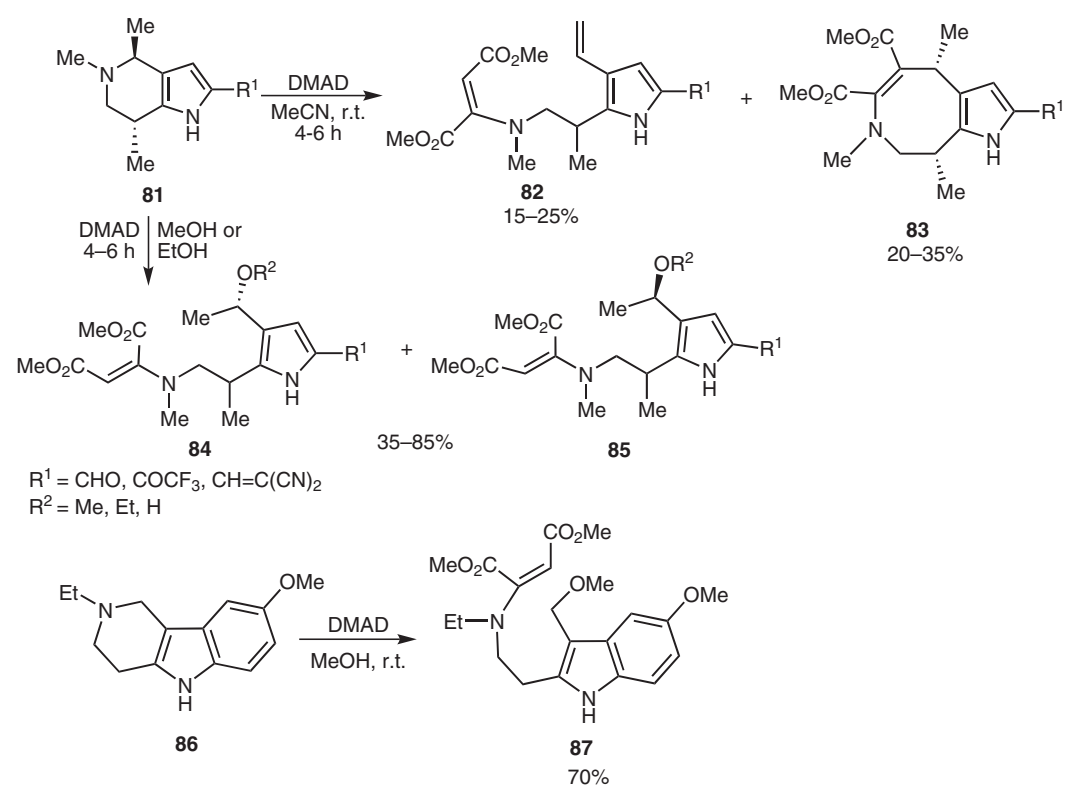

\section{Scheme 22}

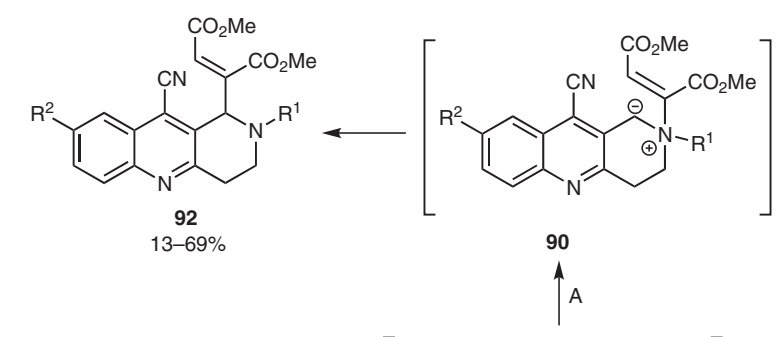

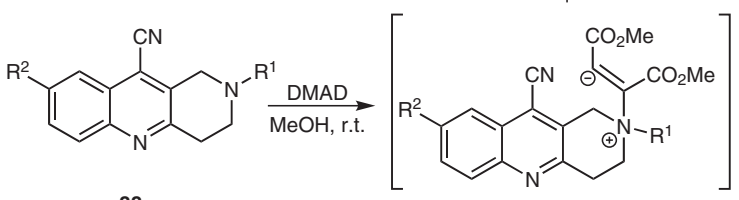

88

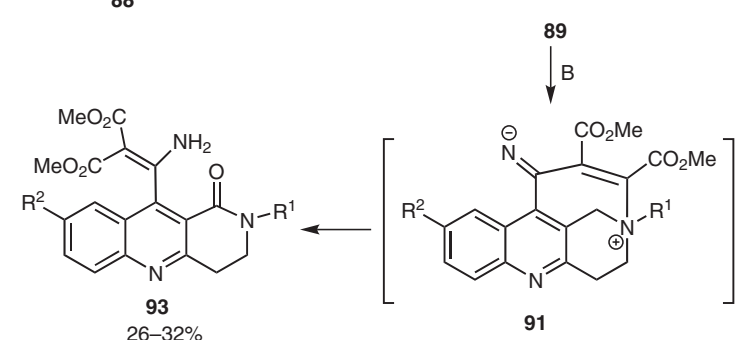

$\mathrm{R}^{1}=i-\mathrm{Pr}, \mathrm{Me}, \mathrm{Bn}$

$\mathrm{R}^{2}=\mathrm{H}, \mathrm{F}, \mathrm{Br}$

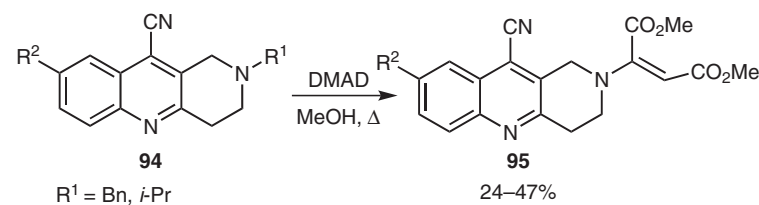

$\mathrm{R}^{1}=\mathrm{Bn}, i-\mathrm{Pr}$
$\mathrm{R}^{2}=\mathrm{H}, \mathrm{Br}$

\section{Scheme 23}

Tetrahydropyridines, $[c]$-condensed with $\pi$-excessive pyrrole, indole, and thiophene units or with a benzene ring, were converted to either condensed azocines ${ }^{26}$ or azonines $^{28}$ under the influence of DMAD. However, in the case of tetrahydropyridines 96, the reaction most probably begins with the addition of the nitrogen atom of the tetrahydropyridine moiety of $\mathbf{9 6}$ to the triple bond of DMAD, resulting in the formation of an ammonium zwitterion leading, finally, to compounds 97, 98 and 99 (Scheme 24). ${ }^{29}$

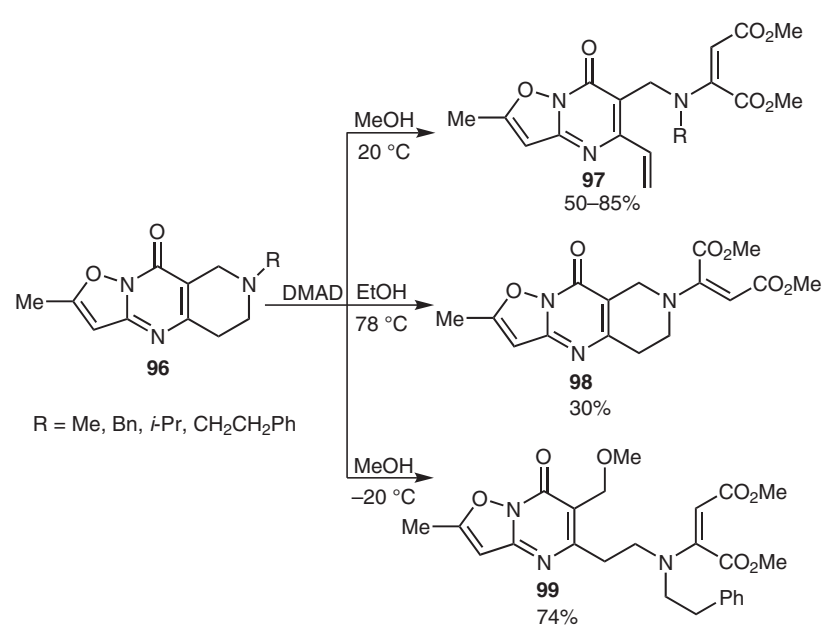

Scheme 24

Analogous opening of the tetrahydropyridine ring was observed by the reaction of thiazolo- and thiadiazolocondensed pyridopyrimidines $\mathbf{1 0 0}$ and 102 with DMAD in methanol, at temperatures from -15 to $-20^{\circ} \mathrm{C}$. These reactions led to the 5-vinyl-substituted thiazolopyrimidines 101 in 56-95\% yields and to the thiadiazolo-pyridopyrimidines 103 in $20-60 \%$ yields, respectively (Scheme $25) .{ }^{29}$ 


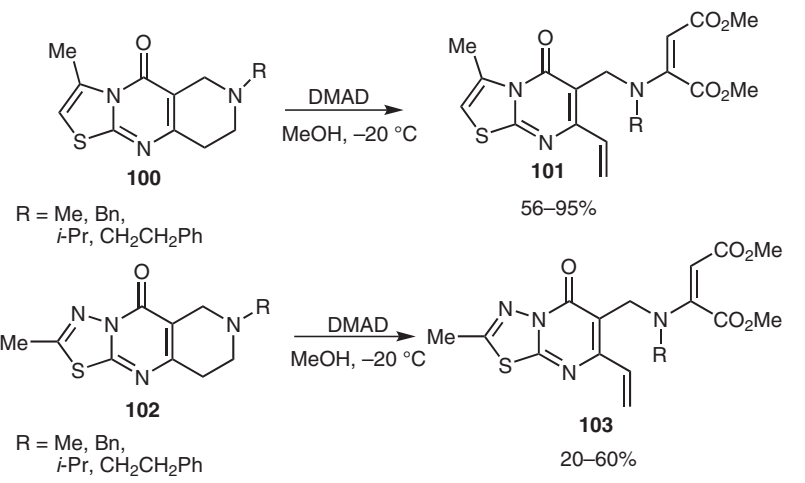

Scheme 25

In 2012, the synthesis of various highly functionalized thiazolopyridines 105, along with the open chain products 106, was accomplished by the reactions of keto-enol tautomeric pairs of heterocycles 104 with DMAD (Scheme 26). ${ }^{30}$

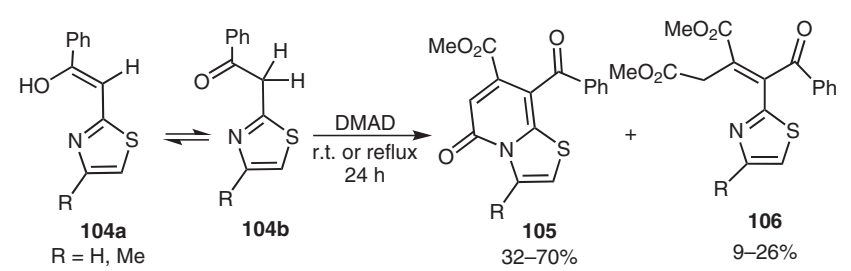

Scheme 26

It was reported that the parent Tröger base $\mathbf{1 0 7}$ reacted with DMAD in the presence of boron trifluoride-diethyl etherate to give compound $\mathbf{1 0 8}$ in $60 \%$ yield (Scheme 27). ${ }^{31}$

Hydrogenated $\gamma$-carbolines 109 underwent tandem piperidine ring cleavage on treatment with DMAD in the presence of alcohols, producing 3-alkoxymethyl-substituted

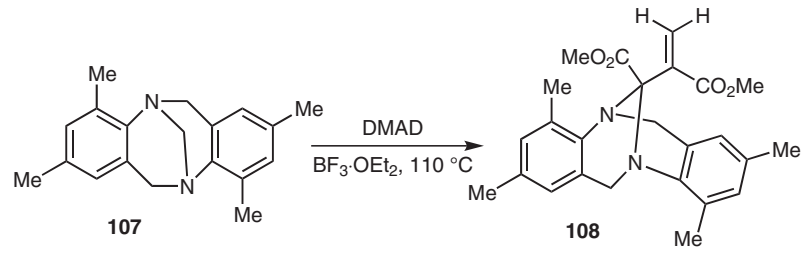

Scheme 27

indoles 112 in good yields. These compounds were cyclized to tetrahydroazocino[4,5- $b$ ] indoles 113 in the presence of aluminum trichloride (Scheme 28). ${ }^{32}$

González-Gómez et al. ${ }^{33}$ reported novel domino reactions in $\beta$-carbolines with DMAD. Vinylpyrrolo- $[2,1-a]-\beta$-carbolines 114 gave different products upon reaction with dienophiles as, with DMAD, a novel domino process took place, involving Michael attack and rearrangement, affording complex polycycles like 115, 116 and 117.

In addition, the reaction of 2-allyl-1-vinyl- $\beta$-carboline 118 with DMAD gave a mixture of products 119 and 120 in a 1:3 ratio, 120 being an unstable product. These products resulted from the same rearrangement reaction. The reaction began with the nucleophilic attack on DMAD, behaving as a Michael acceptor, followed by nucleophilic attack on one unsaturated carbon which led to new polycycles with an increase in skeletal complexity (Scheme 29).

\subsubsection{Reactions with Primary and Secondary Amines}

Zewge et al. ${ }^{34}$ described a mild and efficient synthesis of the quinoline derivatives $\mathbf{1 2 2}$ through a Michael reaction of commercially available aryl amines with DMAD in alcoholic solvents (Scheme 30).

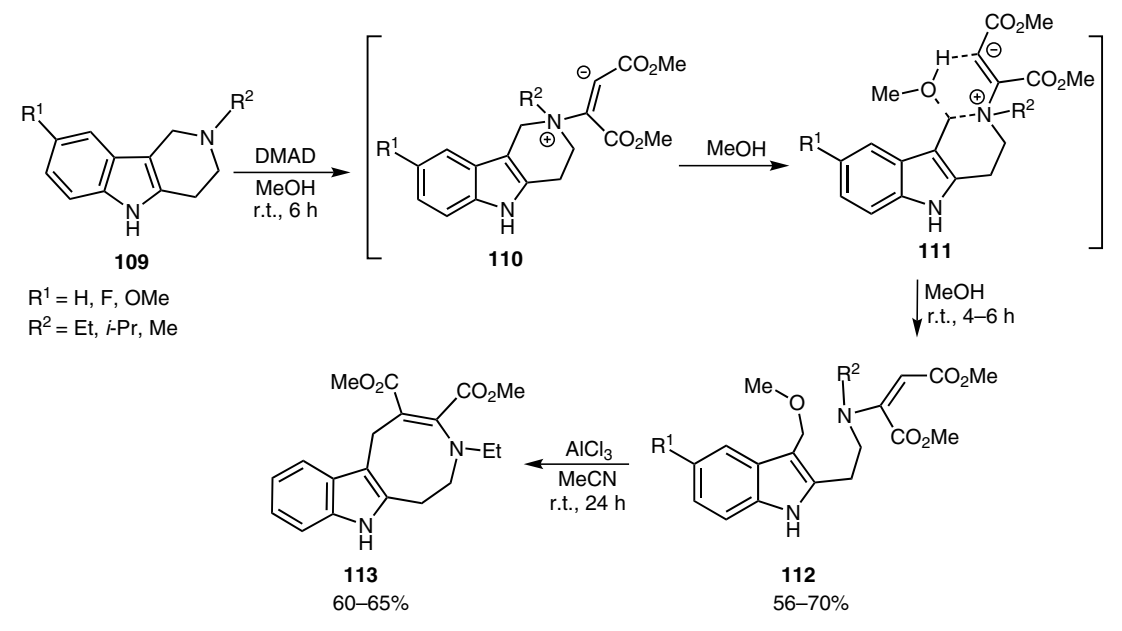

Scheme 28 


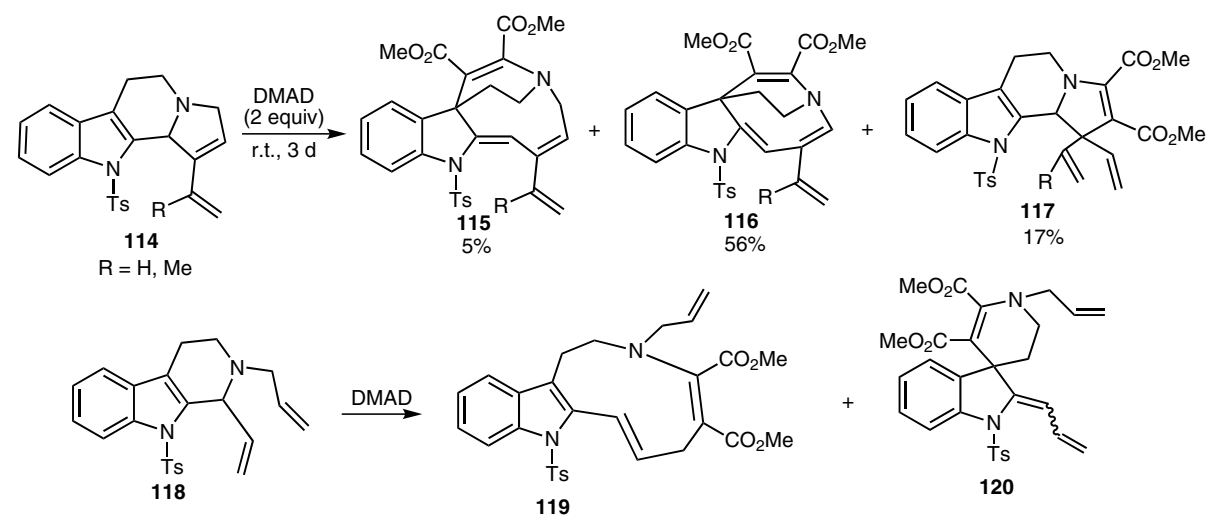

Scheme 29

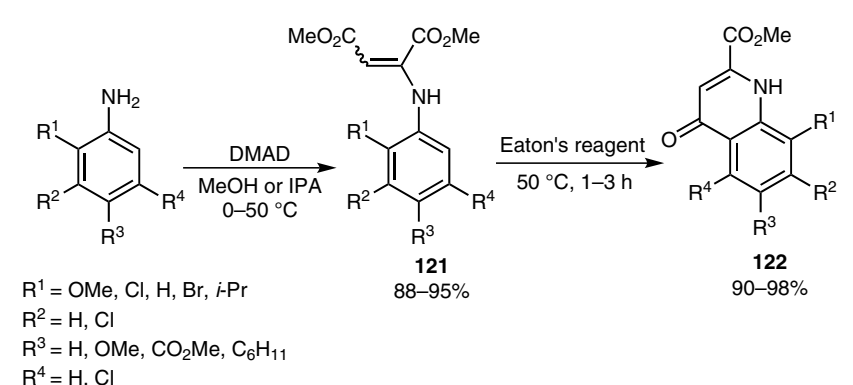

Scheme 30

The reaction of primary and secondary aliphatic amines 123 and 125 with DMAD, for less than two hours, in aqueous medium afforded compounds 124 and 126, respectively (Scheme 31$).^{20}$

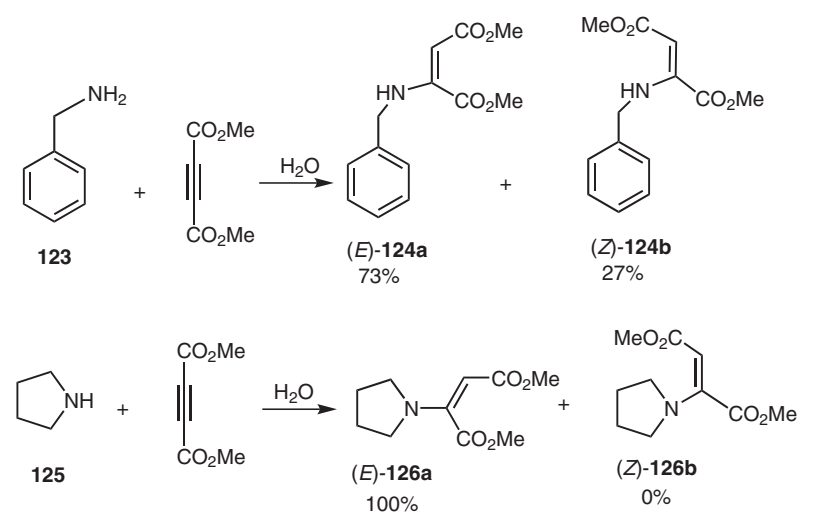

Scheme 31

Reaction of 1,2-diaminocyclohexane (127) with DMAD gave quinoxaline derivatives $\mathbf{1 2 8}$ and/or 129, depending on the molar ratio of the starting materials (Scheme 32). ${ }^{35}$

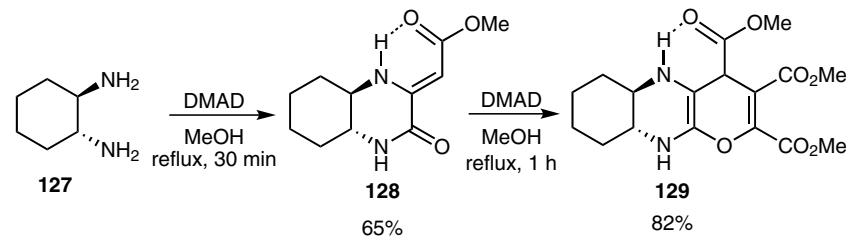

Scheme 32

The reaction of diamine $\mathbf{1 3 0}$ with DMAD afforded the nitrogen-bridgehead pyrido-triazepine 131 (Scheme 33). ${ }^{36}$<smiles>CC(=O)C1C=C(C(=O)Nc2ccccc2)Nc2c(C#N)c(-c3ccc(Cl)cc3)c(C#N)c(=O)n21</smiles>

Scheme 33

In the next example, the initial Michael reaction on nitrogen (amine group) was followed by a reaction with a third component. Ramesh et al. ${ }^{37}$ described a simple and efficient three-component protocol for the synthesis of highly substituted pyrroles 134 by using amines 132, DMAD and glyoxal (133), with DABCO as a catalyst (Scheme 34). Highly functionalized pyrroles were also synthesized by using amines, DMAD, triphenylphosphine and arylglyoxals. $^{38}$

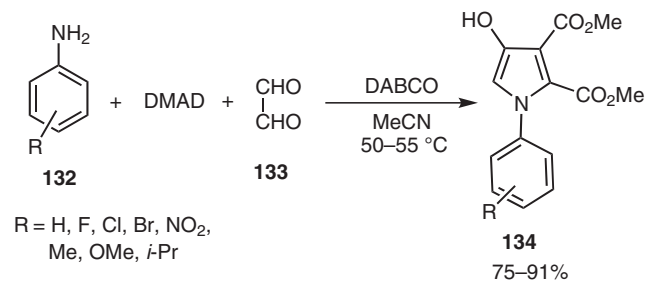

Scheme 34 


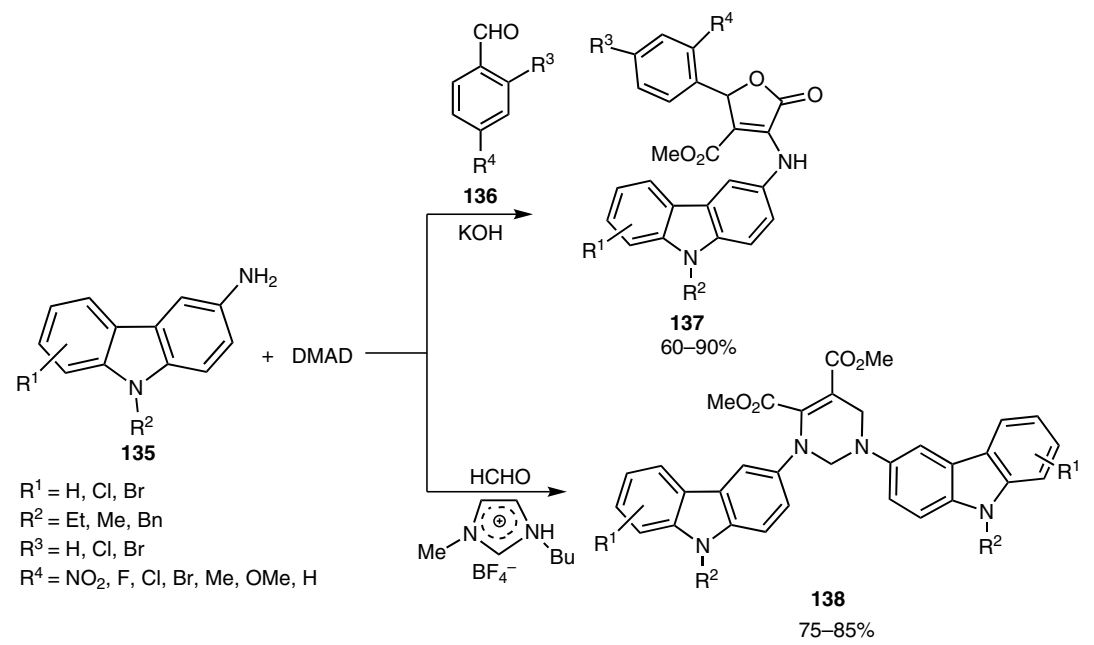

Scheme 35

In 2011, three-component reactions involving 3-aminocarbazoles 135, DMAD and aromatic or aliphatic aldehydes affording carboline derivatives 137 and 138 were described (Scheme 35). ${ }^{39}$

Although the reaction of thiosemicarbazones with DMAD was used for the synthesis of thiazolidinones ${ }^{9}$ (Scheme 5), Vijesh et al..$^{40}$ reported the synthesis of imidazole derivatives 141, containing a substituted pyrazole moiety, by reflux of the thiosemicarbazones 140 with DMAD in methanol (Scheme 36).

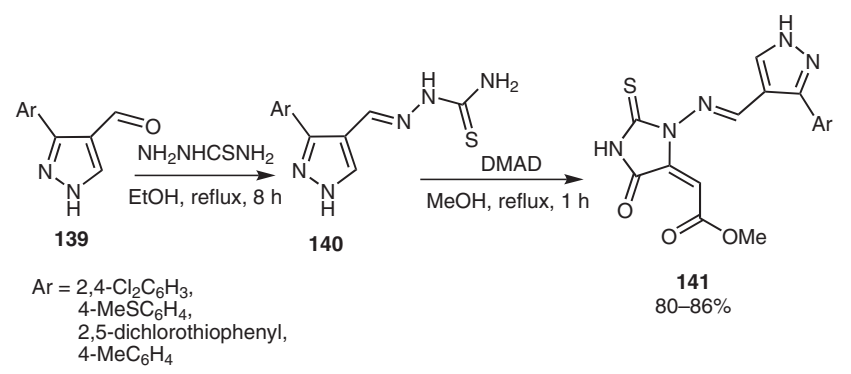

Scheme 36

Moreover, reaction of thiosemicarbazide 142 with DMAD, either in hot methanol or in a solventless system under microwave irradiation (Scheme 37), afforded the triazine derivative $143 .{ }^{41}$<smiles>COC(=O)/C=C1\NNC(=S)NC1=O</smiles>

Scheme 37

The reaction of DMAD with guanidines 144 yielding the five-membered imidazolin-4-ones 145 was also reported (Scheme 38). ${ }^{42}$

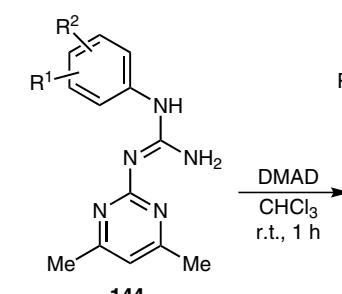

144

$\mathrm{R}^{1}=\mathrm{H}$, 3-Me, 3-MeO, 3-Cl

$\mathrm{R}^{2}=\mathrm{H}, 2-\mathrm{Me}, 4-\mathrm{Me}, 4-\mathrm{MeO}$,

4-EtO, 4-Et, 3-F, 4-F

4- $\mathrm{Ph}, 4-\mathrm{Bu}, 4-\mathrm{Cl}$, 2-MeO

\section{Scheme 38}

The biological activity of 1,4-diazepine derivatives has been widely explored. Zaleska et al. ${ }^{43}$ studied the formation of new tricyclic ring systems of fused 1,4-diazepines. They found that reaction of the zwitterionic compound 146 with DMAD led to the formation of diazepine 149, in $57 \%$ yield, through a Michael reaction (Scheme 39).

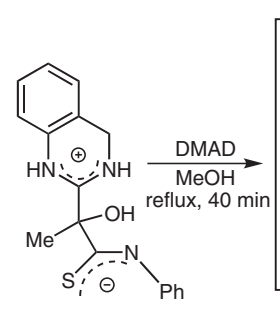

146

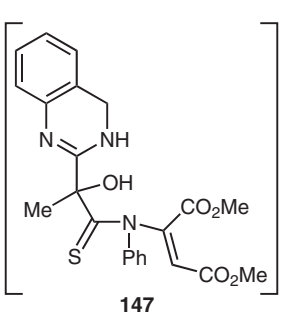

147
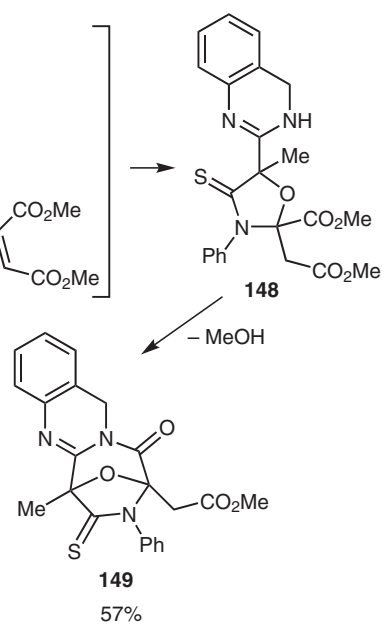

Scheme 39 


\subsection{Oxygen as Nucleophile}

Humphrey et al. ${ }^{44}$ reported that Michael reaction of oxime 151 afforded, through oxygen, a $Z / E$ mixture of adducts 152 (Scheme 40). The final target of this synthesis was an HIV integrase inhibitor.

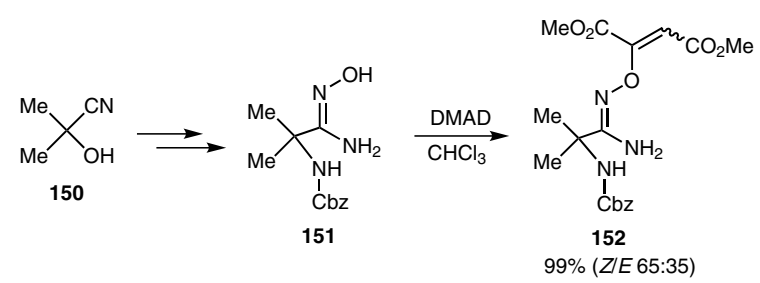

Scheme 40

Furthermore, a gold-catalyzed approach for the regioselective synthesis of highly substituted pyrroles 155 directly from oximes 153 and DMAD was recently developed (Scheme 41). ${ }^{45}$

\subsection{Addition to Carbon-Carbon Double Bonds}

Nitrogen heterocycles containing phosphorus functional groups are compounds of interest in many areas of industrial chemistry such as the textile, pharmaceutical, and agricultural fields. ${ }^{46} \mathrm{~A}$ useful strategy for the preparation of such compounds is based on the cyclization of functionalized enamines. $\beta$-Enamine phosphine oxides were prepared by a one-pot process involving the sequential reaction of triphenylphosphine oxide with methyllithium and then with alkyl and aryl nitriles. The enamines added regioselectively, through the $\beta$-carbon, to the carboncarbon triple bond of DMAD with a stereoselectivity that depended on the substituent of the enamine. Heating the phosphoryl enamines afforded phosphorus-substituted 2pyridones 159 and 2-pyrrolidones 160 in good to excellent yields (Scheme 42 ). ${ }^{47}$

A general and versatile high-yielding method for the divergent and diastereoselective synthesis of polyhydroxylated indolizidines such as $\mathbf{1 6 3}$ has been established using DMAD. At ambient temperature, the reaction of $\mathbf{1 6 1}$ with DMAD proceeded rapidly to form a yellow-colored compound, which was isolated and identified as the adduct 162 (Scheme 43). ${ }^{48}$ In warm methanol, 162 was converted into 5-indolizinone-7,8-dicarboxylate in $88 \%$ yield; this was then itself converted into the polyhydroxylated indolizidine $\mathbf{1 6 3}$ in good yield through practical hydrogenation and reduction reactions.

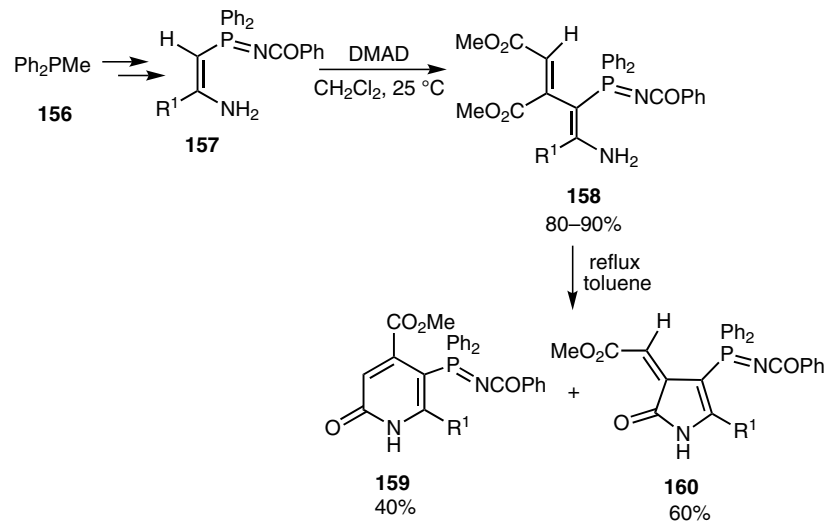

Scheme 42

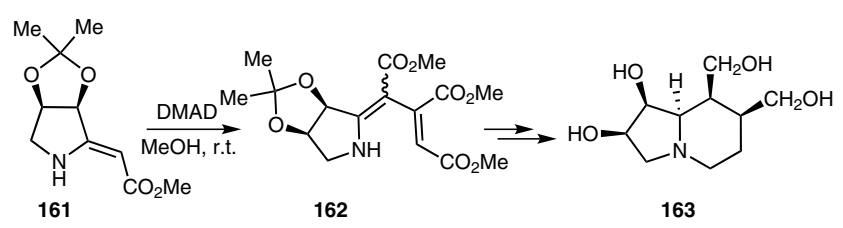

Scheme 43

The indole ring system exists ubiquitously in natural products and many indole-containing compounds exhibit important biological and pharmaceutical activities. $O$ Alkynylanilines 164 reacted with DMAD under the catalysis of platinum(II) chloride to generate the corresponding 2,3-disubstituted indoles 165 (Scheme 44). The reaction proceeded by a sequential cyclization-intermolecular addition pathway. ${ }^{49}$

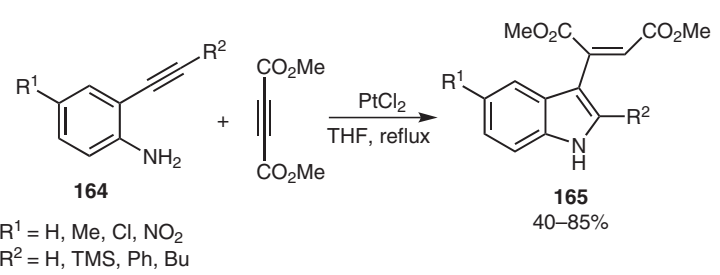

\section{Scheme 44}

Indigotin (166) is the major chemical constituent of indigo, which has been used as a dyestuff for at least 4000 years. The reduced monomeric unit of indigotin, 3-hydroxyindole (167) is much less studied owing to its easy oxidative dimerization to indigotin 166 . The pyrrolone de-

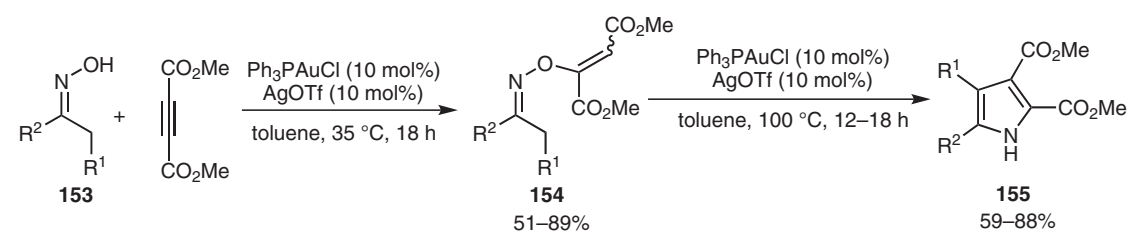

Scheme 41 
rivative 168, a monomeric unit of indigotin 166 with the benzene ring being replaced by a heterocyclic ring, underwent a Michael reaction with DMAD, giving 169 (30\%) as a single isomer. Reactions of 1 -substituted pyrrol$3(2 H)$-ones with DMAD could also take place at the corresponding 2-position, though the tautomeric nature of the product was different (Scheme 45). ${ }^{50}$<smiles>O=C1CNc2ccccc21</smiles>

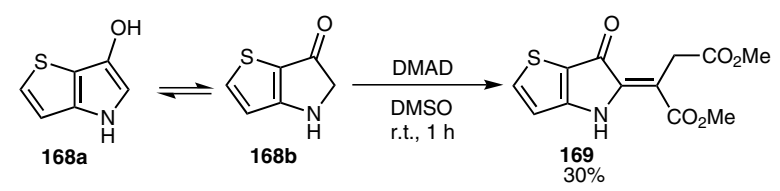

Scheme 45

\section{$3 \quad$ Cycloaddition Reactions}

As previously stated, DMAD is a powerful dienophile widely used in cycloaddition reactions. The most important examples of cycloadditions are the Diels-Alder reactions, in which very often DMAD is used as a standard to check the efficiency of various dienes, and also the 1,3-dipolar cycloaddition reactions. Moreover, [2+2] cycloadditions involving DMAD are also reported. There are also some examples of other cycloadditions, such as [8+2], involving this useful alkyne diester (Scheme 46).

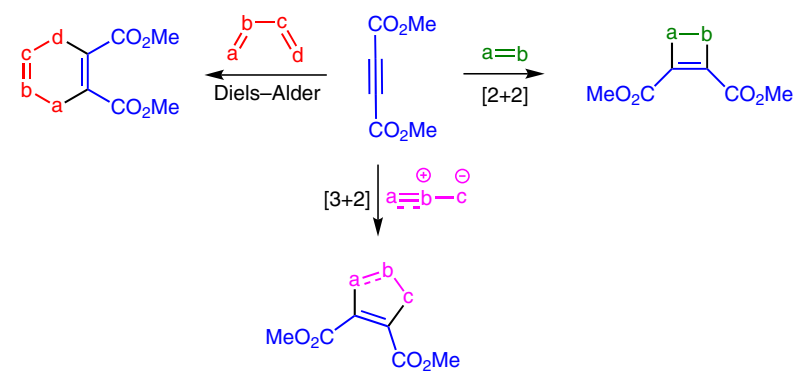

Scheme 46

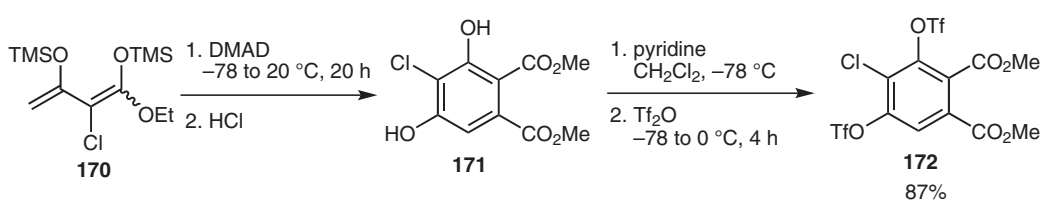

Scheme 47

The reaction of $\mathbf{1 7 6}$ with DMAD afforded a new DielsAlder adduct $\mathbf{1 7 7}$ in 58\% isolated yield (Scheme 49). However, when the reaction was carried out with 1,1,2,2tetracyanoethylene (TCNE) under the same reaction conditions, no reaction was observed. ${ }^{54}$

Dendralenes are cross-conjugated hydrocarbons that quickly became attractive starting materials for organic synthesis. The following example shows a diene-transmissive Diels-Alder addition of DMAD to dendralene 178. The process could be stopped at the mono adduct stage 179, but it could also be performed, under harsher conditions, to yield directly the 2:1 adduct $\mathbf{1 8 0}$ (Scheme 50). ${ }^{55}$ 
<smiles></smiles>

176

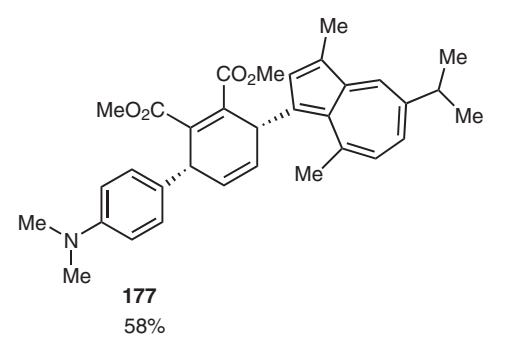

Scheme 49

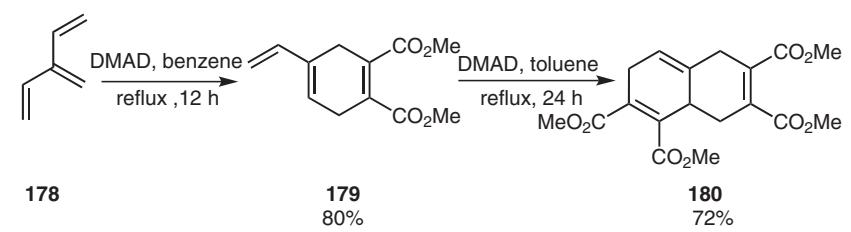

Scheme 50

Ferrocenophane 181, obtained by the ene-yne metathesis method, possessing a conjugated diene functionality in the bridging side chain could be further modified via a DielsAlder cycloaddition with DMAD, in a highly diastereoselective fashion, affording compound 182 (Scheme 51). ${ }^{56}$

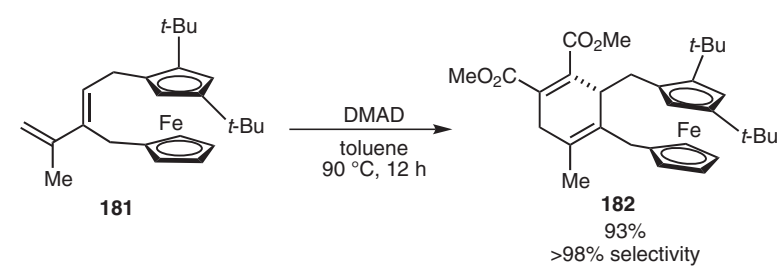

Scheme 51

Nair et al. ${ }^{57,58}$ reported the facile Diels-Alder reaction of compound 183 with DMAD affording a hexasubstituted benzene derivative 184 (Scheme 52).

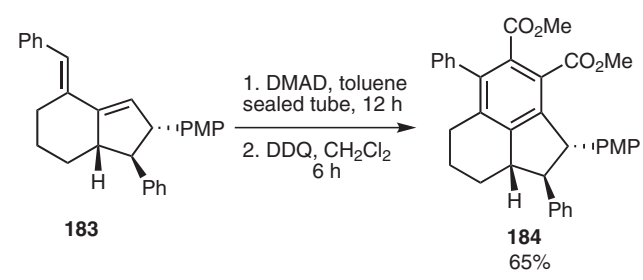

Scheme 52
DMAD is often used in natural product synthesis in key transformation steps. A recent example presenting a stereoselective synthesis of marine sesterterpenes 188 and 189 included a Diels-Alder addition with DMAD (Scheme 53). ${ }^{59}$

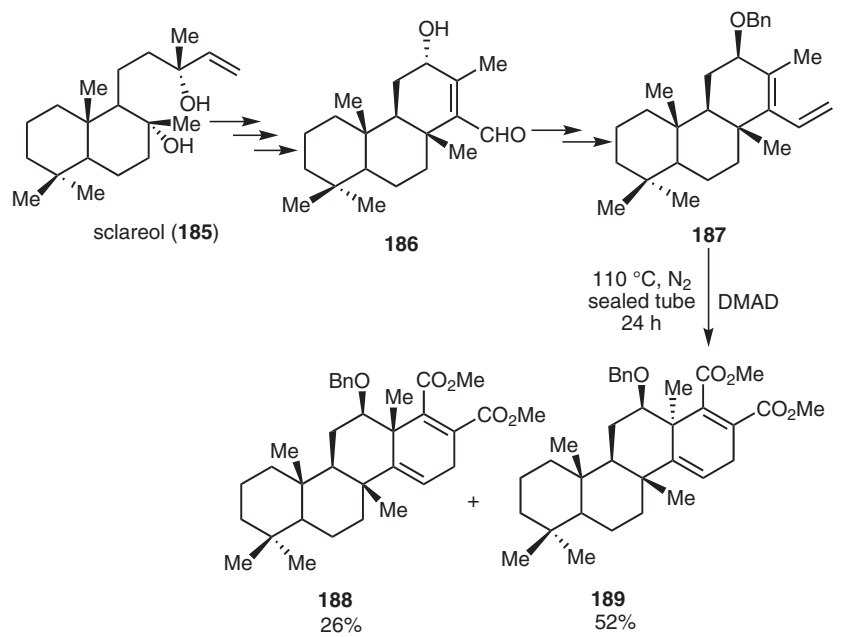

Scheme 53

Sher et al ${ }^{60}$ showed that the reaction of compound 192 with DMAD, in the presence of a catalytic amount of $p$ toluenesulfonic acid (PTSA; 5 mol\%), afforded 193 (Scheme 54).

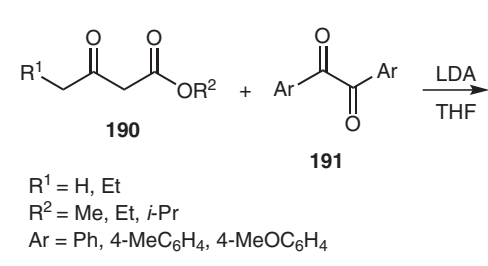

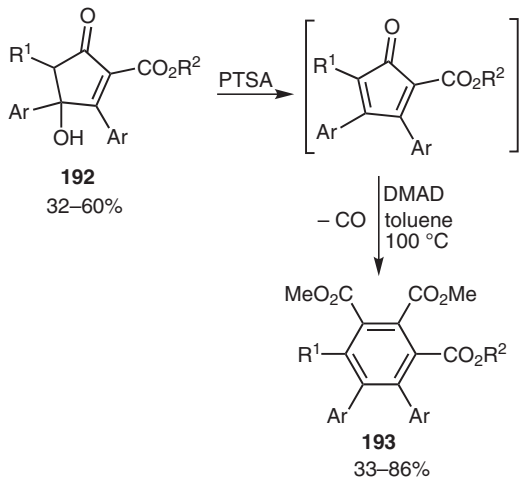

Scheme 54 
The Diels-Alder reaction of $\alpha$-tropolone 194 with DMAD, promoted by triethylamine or silica gel, yielded adduct 195 as reported by Okamura et al. (Scheme 55). ${ }^{61}$

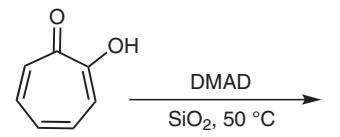

194

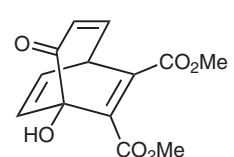

195
$50 \%$
Scheme 55

The synthesis of 198 commenced with 1,6-methano[10]annulene 196, which added DMAD via its ringclosed bis-norcaradiene valence isomer (Scheme 56). ${ }^{62}$<smiles>C1=CCC2(C=C1)C=CC=CC2</smiles><smiles>CC=CC(C)CCCC</smiles>

196
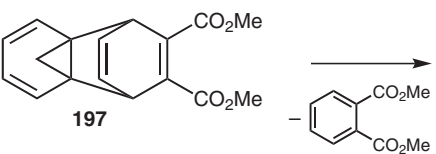

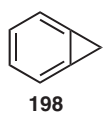

198
Scheme 56

DMAD has also been employed in materials synthesis. Recently, a Diels-Alder reaction of compound 199 with DMAD affording compound 200, a useful intermediate for the synthesis of photoalignment layers for liquid crystals, was reported (Scheme 57). ${ }^{63}$

Allenes, by virtue of their reactive and cumulative double bonds, are excellent partners for both [4+2] and [2+2] cy-
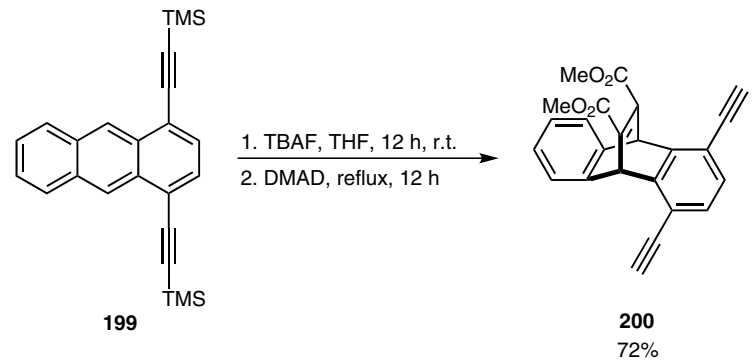

Scheme 57

cloadditions. Allenylphosphonates (phosphorylated allenes) and allenylphosphine oxides constitute a class of compounds that are more readily accessible (and inexpensive) than most of the other allenes. ${ }^{64,65}$ Allenes 201 $(\mathrm{R}=\mathrm{Ph}, p$-tolyl, bromophenyl, $p$-anisyl $)$ with a terminal $=\mathrm{CH}_{2}$ group gave products 202-204. Even more interesting was the reaction of vinyl allenes $\mathbf{2 0 5}$ with three equivalents of DMAD that led to products 206-209 (Scheme 58). ${ }^{66}$

DMAD was also used recently in a transition-metal-mediated annulation in which polyfunctionalized arenes were constructed. This strategy is based on an enyne metathesis followed by Diels-Alder cycloaddition and oxidation. Treatment of diphenylacetylene (210) with the secondgeneration Grubbs catalyst and DMAD, in the absence of additives, gave a mixture of 211, resulting from enyne metathesis, and the non-metathesis product 212 (Scheme 59). ${ }^{67}$

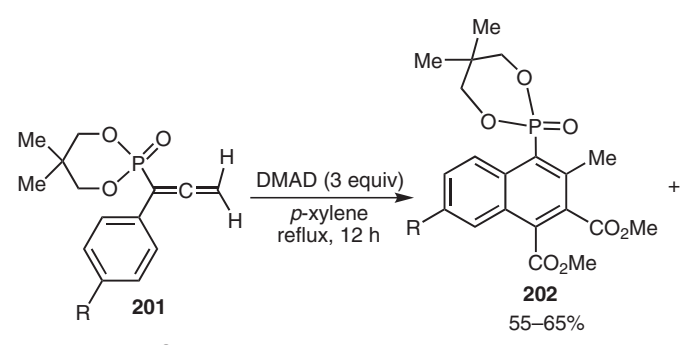

$\mathrm{R}=\mathrm{H}, \mathrm{Me}, \mathrm{Br}, \mathrm{OMe}$

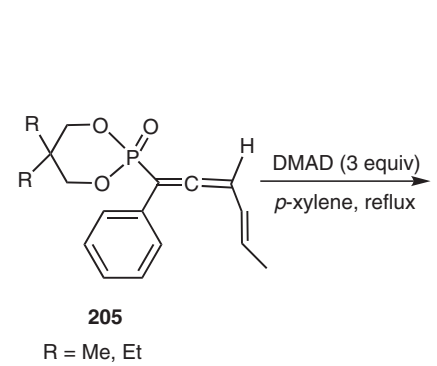

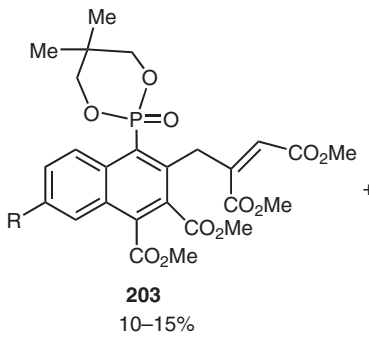

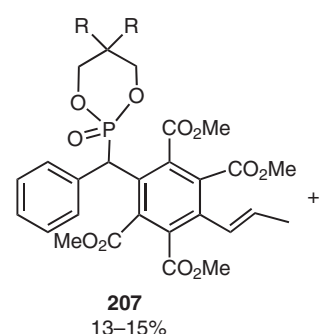

$\stackrel{207}{13-15 \%}$
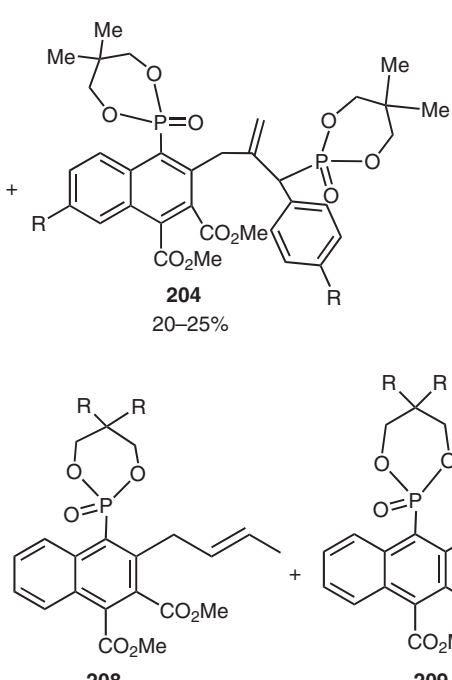

$40-43 \%$

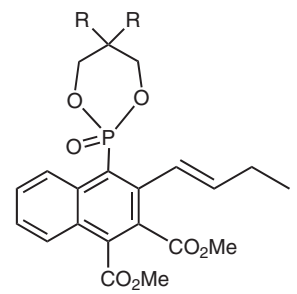

209

Scheme 58 


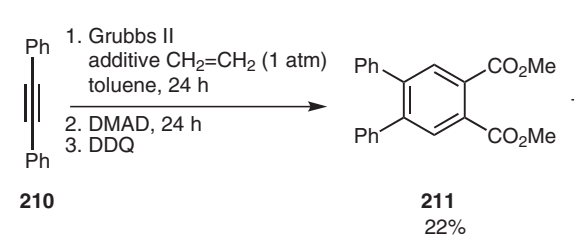

Scheme 59

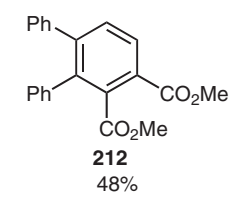

$\%$

\subsubsection{Reactions with o-Quinodimethanes}

The elusive intermediate $o$-quinodimethane, also named $o$-xylylene, has attracted much attention from both theoretical and synthetic chemists over the past 30 years. As cis-dienes, $o$-quinodimethanes have a remarkable DielsAlder reactivity and are often used as building blocks in the syntheses of cyclic organic compounds by inter- or intramolecular [4+2] trapping.

As an example, the efficient procedure for the generation of the imidazole-4,5-quinodimethane intermediate 214, from imidazole derivative $\mathbf{2 1 3}$ and its first-time capture by DMAD to afford the corresponding Diels-Alder benzimidazole adducts $\mathbf{2 1 5}$ and 216, was reported (Scheme 60). ${ }^{68}$

Bicyclic o-quinodimethanes were also prepared from the tricyclic sulfones 217. However, reactions had to be carried out by heating the sulfones with an excess amount of DMAD, in the absence of solvent at $250-320^{\circ} \mathrm{C}$, in order to afford the corresponding cycloadduct 219 (Scheme 61). ${ }^{69}$

Benzopentathiepins 220 reacted slowly with DMAD to give the benzodithiins $\mathbf{2 2 1}$ as the only reaction products, with the reaction being greatly accelerated by the addition of triphenylphosphine. The pentathiepin $\mathbf{2 2 2}$ also reacted with DMAD in the presence of triphenylphosphine in dichloromethane, at room temperature, to give the 1,4-dithiin 223 in $78 \%$ yield. Triphenylphosphine presumably initiates the reaction by nucleophilic attack on sulfur, opening the pentathiepin ring $\mathbf{2 2 0}$ and removing sulfur atoms, possibly to give intermediate $\mathbf{2 2 4},{ }^{70}$ which is intercepted by DMAD (Scheme 62). ${ }^{71}$

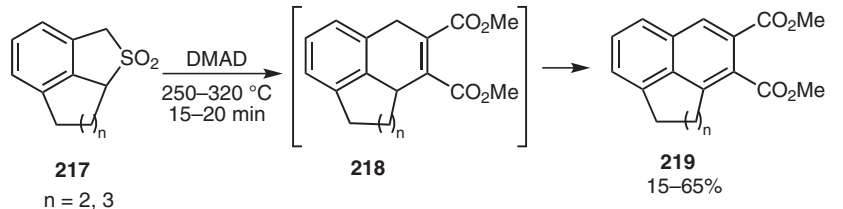

Scheme 61
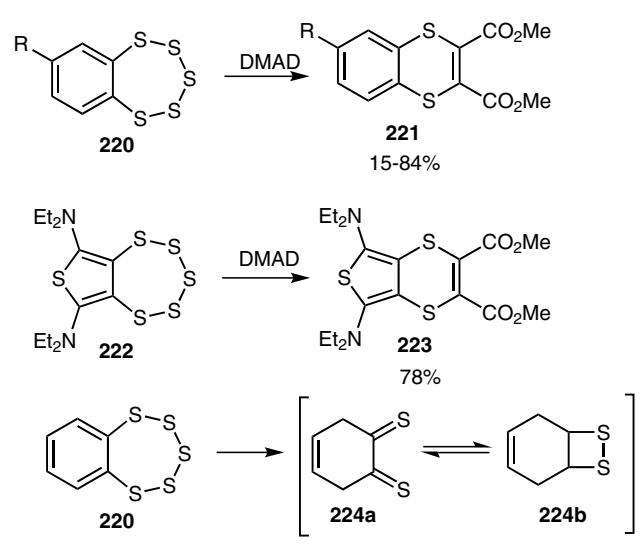

Scheme 62

\subsubsection{Heterocycles as Dienes}

Functionalized arenes $\mathbf{2 2 7}$ were also synthesized through substituted $\alpha$-pyrones 226, which have been used as important synthetic intermediates ${ }^{72}$ and are found in a wide variety of biologically interesting natural substances (Scheme 63). ${ }^{73}$

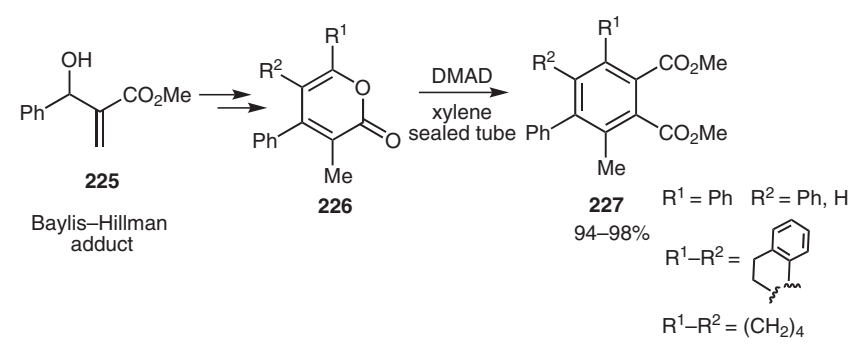

Scheme 63

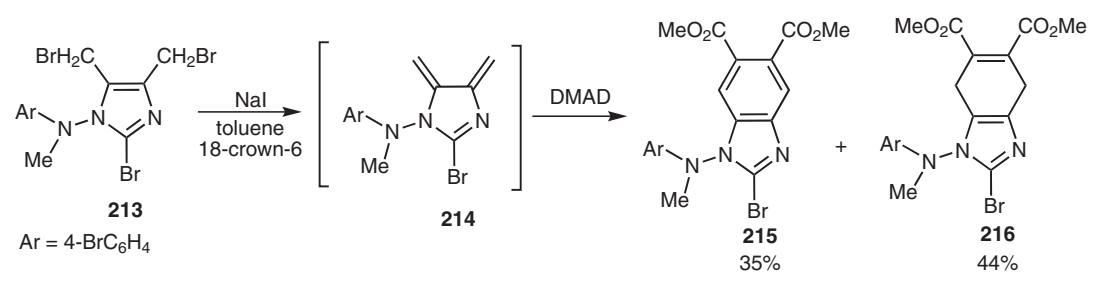

Scheme 60 
Narayan and Sarpong ${ }^{74}$ reported the reaction of DMAD with indolizinone $\mathbf{2 2 8}$, a nitrogen heterocycle recently reported in the literature as a very useful precursor to indolizidine natural products, pharmaceutical agents and new materials. It was supposed to give the product 229; this, however, proved to be unstable and afforded instead the retro-Diels-Alder product 230 (Scheme 64).

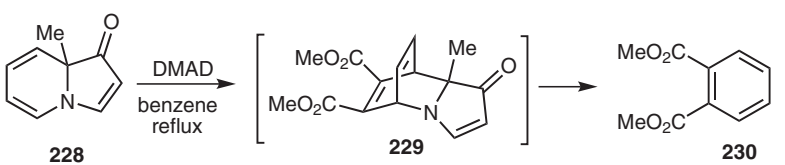

Scheme 64

It has been known for a long time that the reaction of alkynes with heterocyclic dienes, such as furans, results in the formation of bridged oxacycles, which can be transformed into functionalized benzene derivatives by acidic hydrolysis. Diels-Alder reactions of 4-substituted 2-(2furyl)-, 2-styryl-, and 2-crotyl-3-chlorofurans such as $\mathbf{2 3 1}$ with DMAD occurred exclusively on the chlorofurano diene moiety and not on the non-chlorinated furano diene or the chlorinated exocyclic diene alternatives, demonstrating the predominance of the halogen effect in the furan Diels-Alder reaction as shown by Ram and Kumar. ${ }^{75,76}$ For example, chlorobifuryl, having a chlorofuran ring and a non-halogenated furan ring, on heating with DMAD gave exclusively the corresponding furylchlorophenol 233 in $74 \%$ yield by cycloaddition to the chlorofuran ring (Scheme 65).

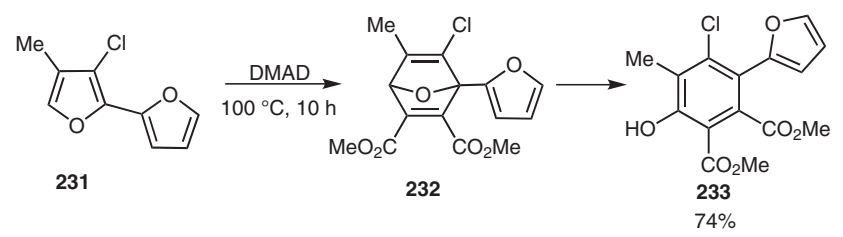

Scheme 65

Moreover, Diels-Alder reactions of furano derivatives such as $\mathbf{2 3 4}$ with DMAD affording functionalized phenols 236 (Scheme 66) have been reported. ${ }^{77}$

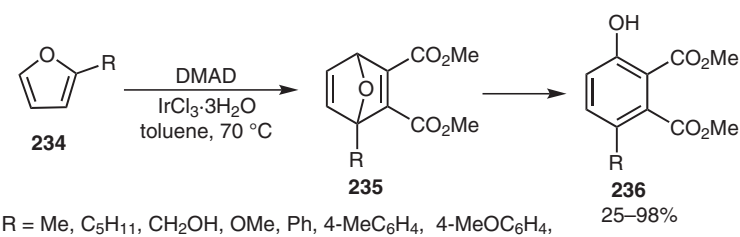

$\mathrm{R}=\mathrm{Me}, \mathrm{C}_{5} \mathrm{H}_{11}, \mathrm{CH}_{2} \mathrm{OH}, \mathrm{OMe}, \mathrm{Ph}, 4-\mathrm{MeC}_{6} \mathrm{H}_{4}, 4-\mathrm{MeOC}_{4} \mathrm{H}^{2}$
$4-\mathrm{O}_{2} \mathrm{NC}_{6} \mathrm{H}_{4}, 4-\mathrm{NCC}_{6} \mathrm{H}_{4}, 4-\mathrm{F}_{3} \mathrm{CC}_{6} \mathrm{H}_{4}, 4-\mathrm{FC}_{6} \mathrm{H}_{4}, \mathrm{CO}_{2} \mathrm{Me}$

\section{Scheme 66}

Furthermore, the reaction of DMAD at the furan fragment of 3,4-fused 2-furyltetrahydroquinoline derivatives $\mathbf{2 3 7}$ according to the [4+2] cycloaddition pattern was studied. The reaction proved not to be stereoselective, yielding two diastereoisomeric 7-oxabicyclo[2.2.1] hepta-2,5-dienes 238a, b in $32-88 \%$ total yield (Scheme 67 ). ${ }^{78}$

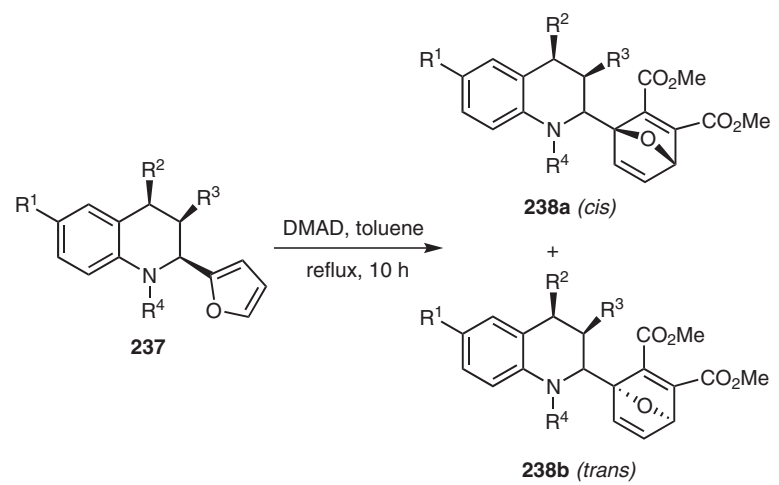

Scheme 67

Reactions of DMAD with pyrroles have been studied extensively. The [4+2] cycloaddition between pyrroles and dienophiles has been shown to be a general method for the synthesis of 7-azabicyclo[2.2.1]hepta-2,5-diene and 7-azabicyclo[2.2.1] hept-2-ene derivatives. However, pyrrole is a poor diene for the $[4+2]$ cycloaddition and usually reacts with DMAD to give Michael addition products. Moreover, when a pyrrole nitrogen atom bears an electron-withdrawing group, the aromatic ring was found to be more reactive as a diene toward DMAD. ${ }^{26}$ Nevertheless, application of ultrasound to the reaction of pyrrole 239 with DMAD in an aqueous solution resulted in the cycloaddition adduct $\mathbf{2 4 0}$ in $60 \%$ yield without the formation of Michael-type products (Scheme 68). ${ }^{79}$

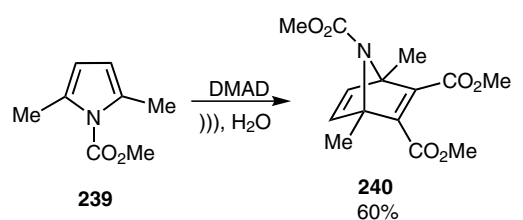

Scheme 68

The [4+2] cycloaddition of 1-(alkylamino)pyrroles or 1(alkoxycarbonylamino)pyrroles with electron-deficient alkynes was shown to follow a predictable pathway and provide a remarkably simple route for the preparation of substituted benzenes. Upon heating 241 with three equivalents of DMAD, benzene derivatives $\mathbf{2 4 3}$ were obtained in good yields (50-90\%; Scheme 69). ${ }^{79}$

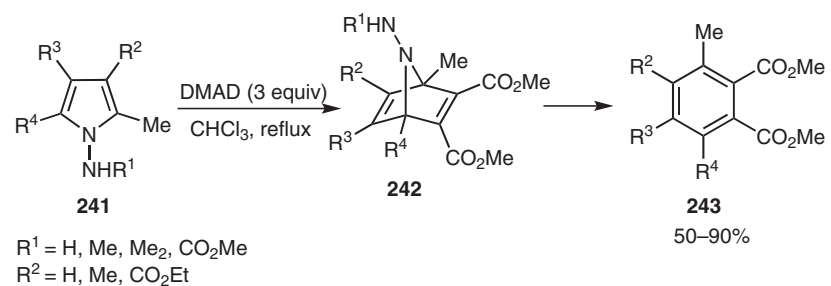

$\mathrm{R}^{2}=\mathrm{H}, \mathrm{Me}, \mathrm{CO}_{2} \mathrm{Et}$

$\mathrm{R}^{3}=\mathrm{H}, \mathrm{Me}, \mathrm{Et}$

\section{Scheme 69}


Pyrrolo[3,4- $b]$ pyrrole 244 afforded the cycloadduct 245 on treatment with DMAD. Oxidation of the latter with $m$ chloroperbenzoic acid, followed by thermolysis, gave the indole derivative 246 (Scheme 70). ${ }^{80}$

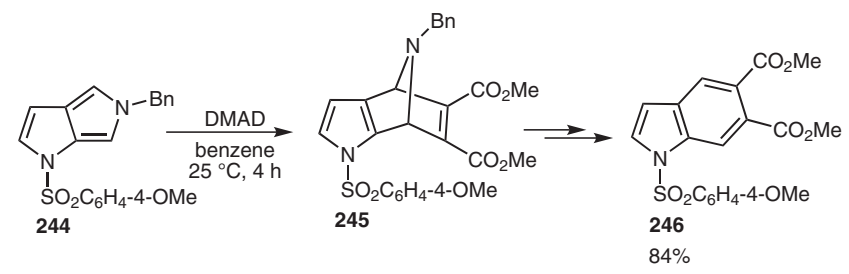

Scheme 70

\subsubsection{Heterocycles with an Exocyclic Double Bond}

Ikeuchi et al. ${ }^{81}$ described a ' $\mathrm{Cp}_{2} \mathrm{Zr}$ '-mediated reaction and subsequent copper(I)-catalyzed carbon-carbon bond formation for the construction of biologically attractive molecules such as compounds 250. In order to examine the reaction's utility, preliminary reactions of the latter compounds to obtain polycyclic heterocyclic systems were examined (Scheme 71). To this end, the reaction of the allylation product 248 gave the unstable diene 249 in good yield, through intramolecular enyne metathesis ${ }^{82}$ using the second-generation Grubbs catalyst (ambient temperature for 15 hours); $\mathbf{2 4 9}$ was then used directly for the subsequent Diels-Alder reaction with DMAD at ambient temperature for 10 hours leading to compound $\mathbf{2 5 0}$ as a major product $(\mathbf{2 5 0} / \mathbf{2 5 1}=24: 1$ in the case of $X=\mathrm{NMe})$.

Interaction of pyrrole $\mathbf{2 5 2}$ with DMAD afforded, after exposing adduct 253 to air, the hydroxyindole 254 and the bis-adduct 7-vinylindole 255 (Scheme 72). The latter compound was formed as a result of the 1,2-addition of the primary cycloadduct 253 to a second DMAD molecule followed by elimination of trimethylsilanol. When the reaction was performed without solvent, under an oxygen atmosphere, indole 254 was mainly formed $(\mathbf{2 5 4} / \mathbf{2 5 5}=$ $72: 28) .{ }^{83}$

Indoles $\mathbf{2 5 7}$ and $\mathbf{2 5 9}$ were obtained, in $17 \%$ and $27 \%$ yield respectively, by reaction of 3-vinylpyrroles $\mathbf{2 5 6}$ and $\mathbf{2 5 8}$ with DMAD (Scheme 73). ${ }^{83 \mathrm{c}}$

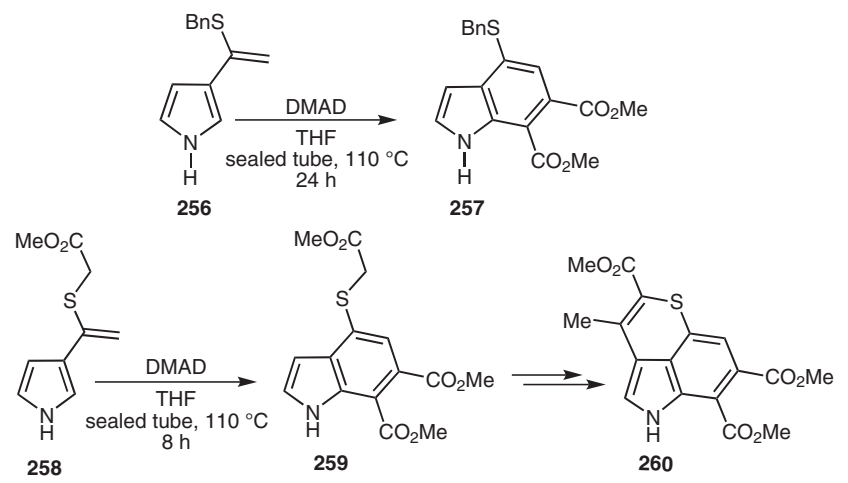

Scheme 73

2-Vinylpyrrolo[2,3- $b$ ]pyridine $\mathbf{2 6 1}$ afforded, upon reaction with 1.5 equivalents of DMAD, compound 262, the unexpected derivative 263, and trace amounts of an aromatized derivative from 262. Attempts to carry out the cycloaddition reaction under atmospheric pressure gave solely $\mathbf{2 6 2}$ in $30 \%$ yield. Cycloaddition of $\mathbf{2 6 1}$ to DMAD (5 equiv) led to compound $\mathbf{2 6 3}$, in low yield, due to the decreased reactivity of the diene ( $22 \%$ of the starting material was recovered) and the formation of cyclodimer 264, resulting from an intermolecular hetero-Diels-Alder reac-

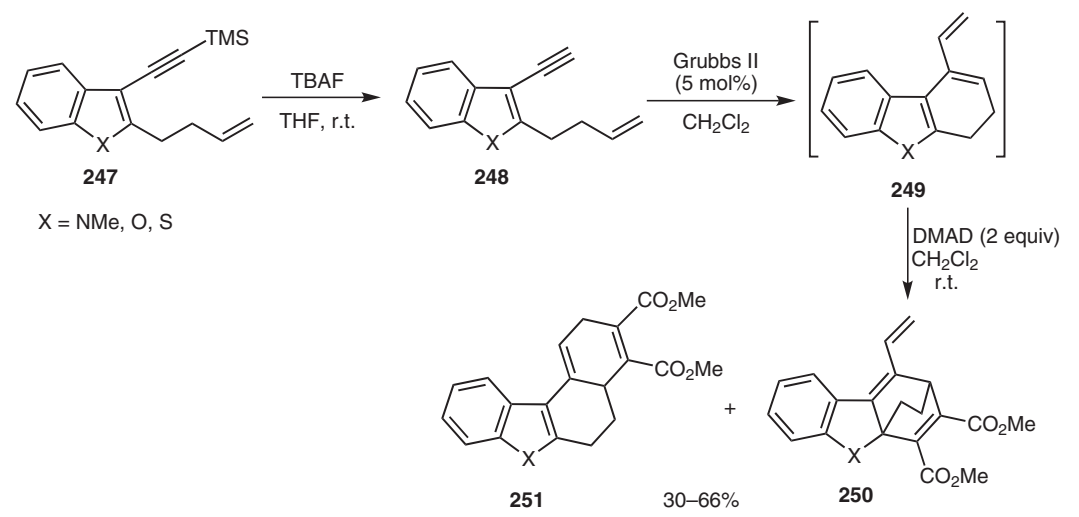

Scheme 71

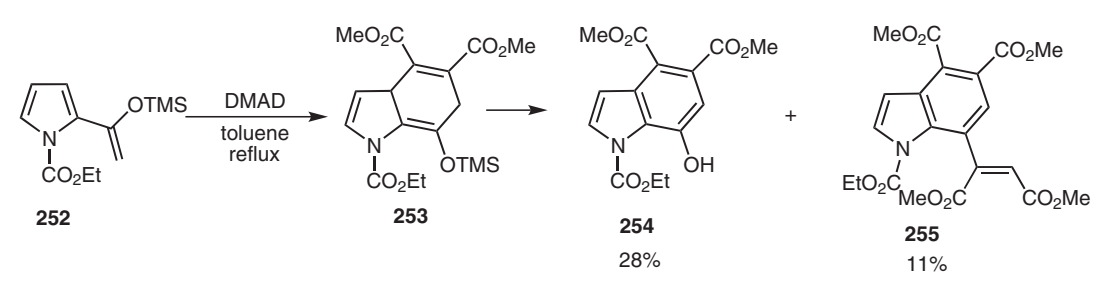

Scheme 72 


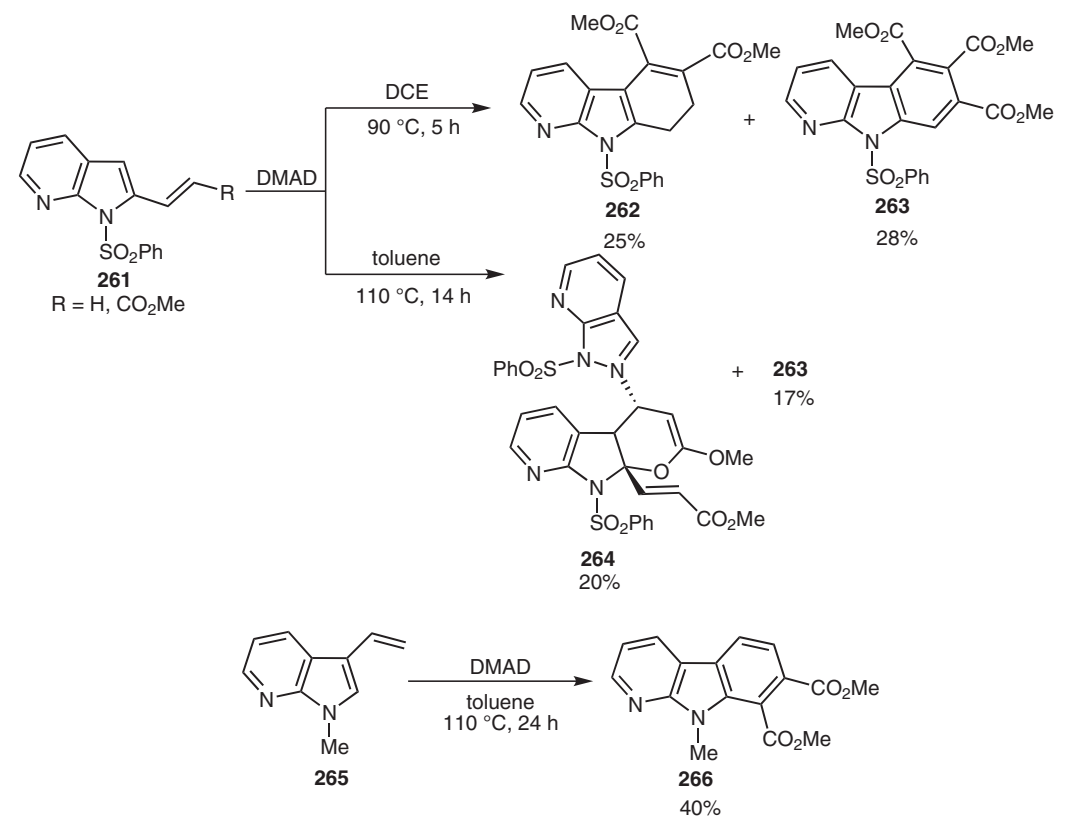

Scheme 74

tion of 261 with itself. Similarly, 3-vinylpyrrolo[2,3$b$ ]pyridine 265 was treated with DMAD (1.2 equiv) to give the indole derivative 266 (Scheme 74). ${ }^{83 \mathrm{~d}}$

\subsubsection{Hetero-Diels-Alder Reactions}

The reaction of equimolar amounts of enaminones ${ }^{84} \mathbf{2 6 7}$ with DMAD resulted to the formation of a variety of pyran and pyrrole-3-ylidene derivatives 268 and $\mathbf{2 6 9}$ (Scheme 75) having pharmacological and medicinal significance. The mechanism concerning pyran derivatives 268 involves an initial [4+2] cycloaddition followed by a 1,3-hydride shift, whereas for $\mathbf{2 6 9}$ an initial enamine-type nucleophilic attack of the $\alpha$-carbon of $\mathbf{2 6 7}$ at the acetylenic carbon of DMAD is proposed. ${ }^{85}$

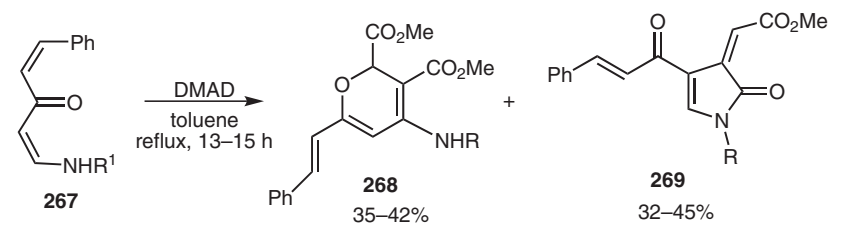

$\mathrm{R}=\mathrm{Ph}, 4-\mathrm{MeOC}_{6} \mathrm{H}_{4}, 4-\mathrm{MeC}_{6} \mathrm{H}_{4}$

\section{Scheme 75}

Compound 270 reacted with DMAD under microwave conditions to yield the pyridine derivative $\mathbf{2 7 3}$, most likely formed via intermediate cycloadduct 271. The latter underwent retroaddition via loss of methylene aniline to yield 272, the conversion of which, under the reaction conditions, led to 273 (Scheme 76). ${ }^{86}$
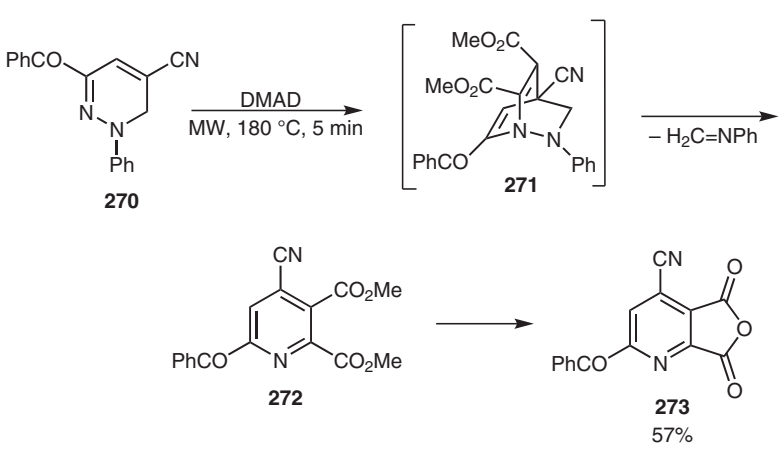

Scheme 76

The reaction of thiazoles 274 with DMAD gave pyridines 275 through a hetero-Diels-Alder reaction and further sulfur extrusion, ${ }^{87}$ although, in this case, extremely harsh conditions were required. The activating effect of the amino functionality in the reaction of 2-(dimethylamino)thiazole toward electron-poor reagents, such as DMAD, tosyl isocyanate, and ketenes has been also demonstrated. ${ }^{88}$ Nevertheless, no cycloadducts were formed in these reactions, but only Michael-type products resulting from the functionalization at the 5-position of the thiazole ring. This activating effect was evident when 2-amino-4-methylthiazole (276) was allowed to react with DMAD. From this reaction, conducted at room temperature, a new adduct $\mathbf{2 7 7}$ was isolated in $42 \%$ yield, along with the thiazolopyrimidinone 278 formed in $30 \%$ yield. The formation of 277 was explained as the result of a [4+2] cycloaddition in which the heterocycle acts as heterodiene (Scheme 77)..$^{87 b}$ 
<smiles></smiles>

274

$\mathrm{R}=\mathrm{H}$, alkyl, aryl

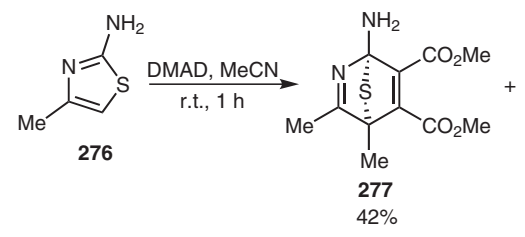

Scheme 77

\subsection{1,3-Dipolar Reactions ([3+2] Cycloadditions)}

Although 1,3-dipoles have been known for more than a century, their cycloaddition reactions are nowadays powerful synthetic tools, providing access to highly functionalized oxygen-, sulfur- and nitrogen-containing heterocycles. The synthetic utility of these cycloadditions has been further enhanced by the development of tandem processes that allow the preparation of complex molecules starting from relatively simple materials.

\subsubsection{Azomethine Ylides}

Azomethine ylides have become one of the most investigated classes of 1,3-dipoles. ${ }^{89}$ The substituted pyrroles $\mathbf{2 8 0}$ were obtained by a one-pot reaction between quinazolinonium bromides 279 and DMAD in 1,2-epoxybutane as the reaction medium and acid acceptor (Scheme 78). ${ }^{90}$

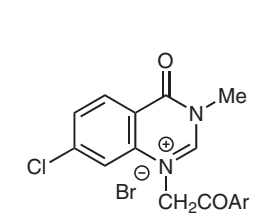

279

$\mathrm{Ar}=\mathrm{Ph}, 3-\mathrm{O}_{2} \mathrm{NC}_{6} \mathrm{H}_{4}, 4-\mathrm{BrC}_{6} \mathrm{H}_{4}$,

4- $\mathrm{MeOC}_{6} \mathrm{H}_{4}, 4-\mathrm{ClC}_{6} \mathrm{H}_{4}$

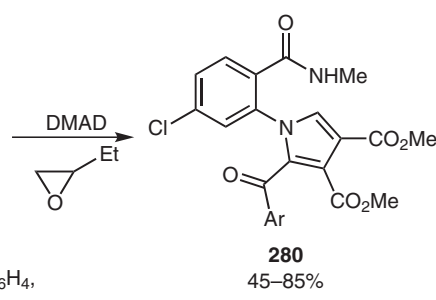

Bridgehead nitrogen heterocycles are important natural products. Among them, indolizines have received much attention in recent years owing to both their intriguing molecular structures featuring $10 \pi$-delocalized electrons and their important biological activities. These molecules have been used in various pharmaceutical applications. The synthesis of indolizine $\mathbf{2 8 2}$ in 53\% yield, via the 1,3dipolar cycloaddition between pyridinium bromide $\mathbf{2 8 1}$ and DMAD, using Amberlite IRA-402 (OH) ionexchange resin as a base, was recently described (Scheme 79). ${ }^{91,92}$

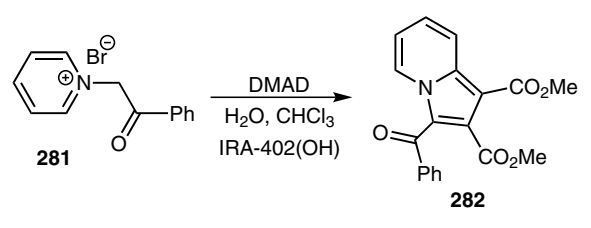

Scheme 79

1,3-Dipolar cycloaddition of $\mathbf{2 8 5}$ with DMAD as dipolarophile yielded the pyrazole derivative $\mathbf{2 8 6}$ in low yield. Attempts to improve the yield by insertion of a spacer yielded pyrazoles $\mathbf{2 8 7}$ and $\mathbf{2 8 8}$ as a result of overreaction in a Michael fashion with DMAD. The polymer-supported azomethine imines which were generated from polymer-supported silylnitrosoamides by a 1,4-silatropic shift gave pyrazole derivatives. A feature of this reaction is that no cleavage operations are required after the cycloaddition. Thus, azomethine imine 290, which was generated via a 1,4-silatropic shift of the silyl group onto the oxygen of the nitroso group, underwent 1,3-dipolar cycloaddition with DMAD to give the five-membered-ring adduct 291. It is interesting that the acyl group was spontaneously eliminated as a silyl ester and N-unsubstituted pyrazoles 292 were obtained after aromatization. If a polymer is attached to the acyl group of $\mathbf{2 8 9}$, the versatility of the reaction is greatly enhanced (Scheme 80). ${ }^{93,94}$

Scheme 78

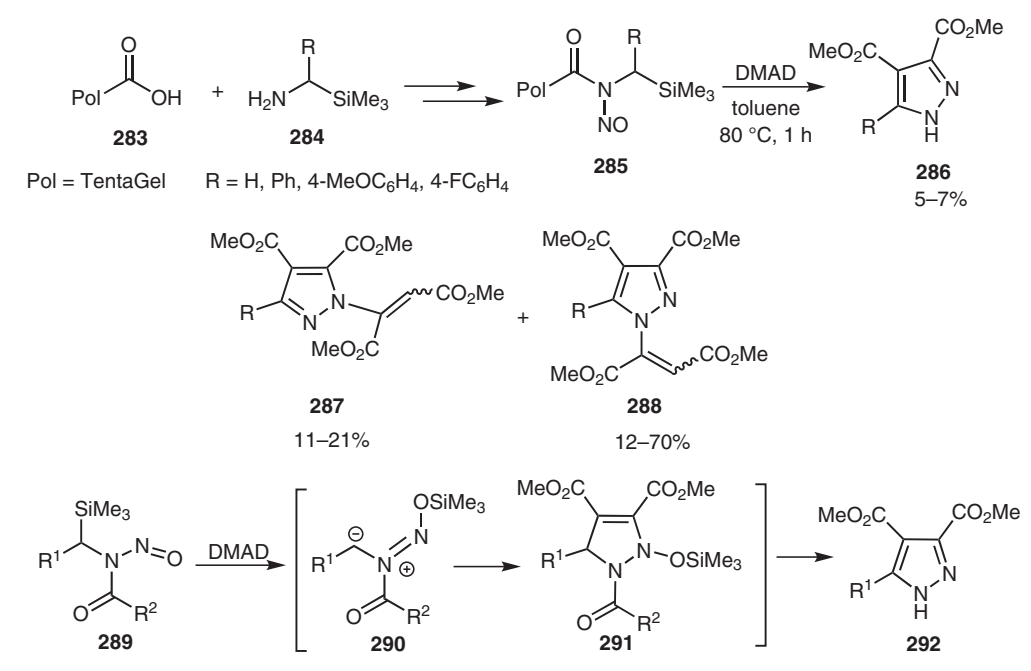

Scheme 80 


\subsubsection{Nitrones}

Mlostoń et al. ${ }^{95}$ described a new method for the preparation of 1,4,5-trisubstituted (imidazol-2-yl)acetates 296, based on the reaction of the corresponding imidazole 3oxides 293 with DMAD. Formation of the products was rationalized by a formal 1,3-dipolar cycloaddition and subsequent oxaloyl cleavage (Scheme 81).

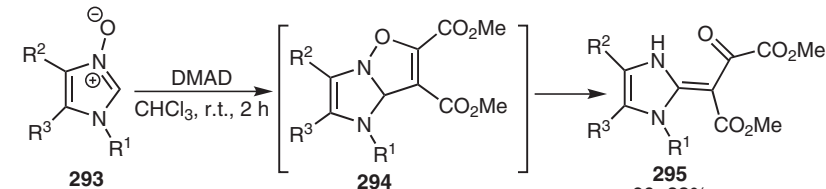

$$
\begin{aligned}
& R^{1}, R^{2}, R^{3}=\text { alkyl, aryl }
\end{aligned}
$$

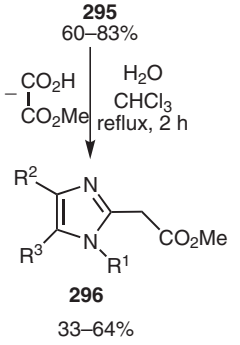

\section{Scheme 81}

Chakraborty and Luite ${ }^{96}$ reported the cycloaddition of $\mathrm{N}$ benzyl fluoro nitrones $\mathbf{2 9 8}$ with DMAD, accelerated by ionic liquids, to afford novel isoxazoline and isoxazolidine derivatives 299 in a one-pot reaction process (Scheme 82).

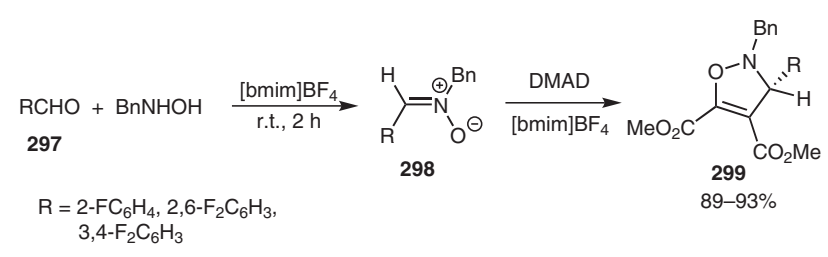

\section{Scheme 82}

\subsubsection{Azides}

The 1,3-dipolar cycloaddition reaction between alkynes and azides, developed by Huisgen, ${ }^{97}$ is one of the most popular reactions because of the resulting five-membered substituted 1,2,3-triazole heterocyclic ring. It was found to have a wide range of industrial applications such as for dyes, photostabilizers and agrochemicals and also in the designing of new drugs. Adducts of azidomethylamines 300 and 302 with DMAD afforded compounds 301 and 303, respectively (Scheme 83). ${ }^{98}$

In 2009, a fast one-pot, microwave-assisted, solvent-free and high-yielding synthesis of dimethyl $1 H$-1,2,3-triazole-4,5-dicarboxylate (305) by 1,3-dipolar cycloaddition reaction with trimethylsilyl azide 304 and DMAD was described (Scheme 84). ${ }^{99}$

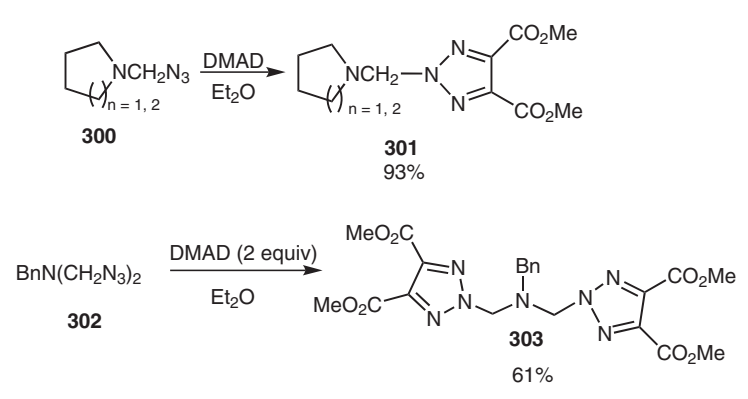

Scheme 83

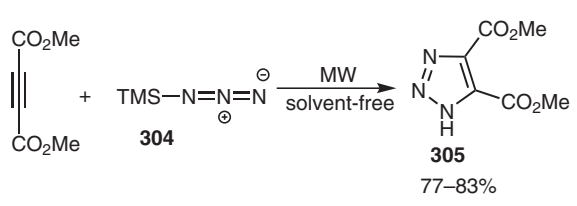

Scheme 84

Kumar and Rode ${ }^{100}$ reported the first general approach for the synthesis of fused 1,2,3-triazolo- $\delta$-lactams $\mathbf{3 0 9}$ using a Huisgen [3+2]-dipolar cycloaddition reaction in water, between activated alkynes and azides such as $\mathbf{3 0 7}$ derived from different amino acids, this 'click' reaction was followed by cyclization (Scheme 85).

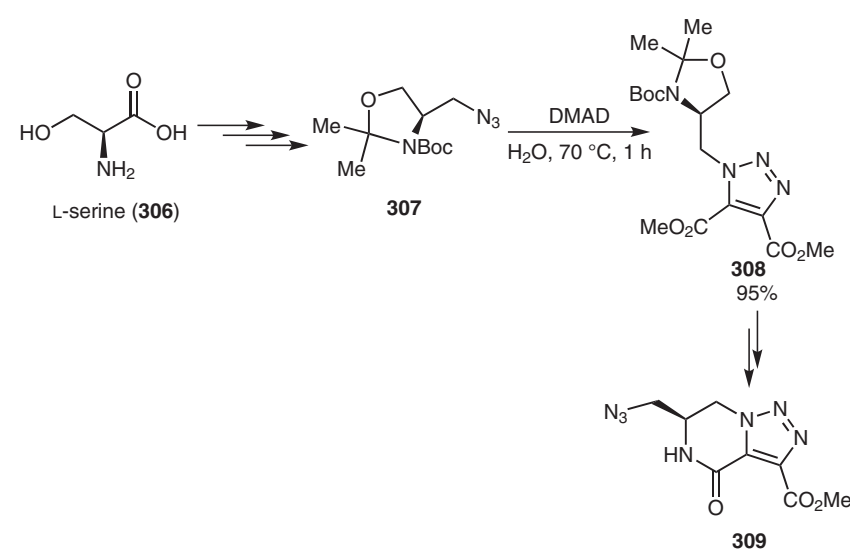

Scheme 85

Semakin et al. ${ }^{101}$ reported that $\alpha$-azidooximes, readily obtained from aliphatic nitro compounds, were cleanly converted into previously unknown pyrazinones 313. Here, oximes 310 reacted with DMAD via [3+2] cycloaddition at room temperature affording triazoles 311. However, the cycloaddition was accompanied by partial hydrolysis of the oximino group leading to carbonyl derivatives 312 . This side process could be avoided by carrying out the $[3+2]$ cycloaddition of $\mathbf{3 1 0}$ with DMAD in toluene. Oximes 311 were readily reduced with Raney nickel when $R^{1}$ was not an ester giving the target heterocycles $\mathbf{3 1 3}$ (Scheme 86). 


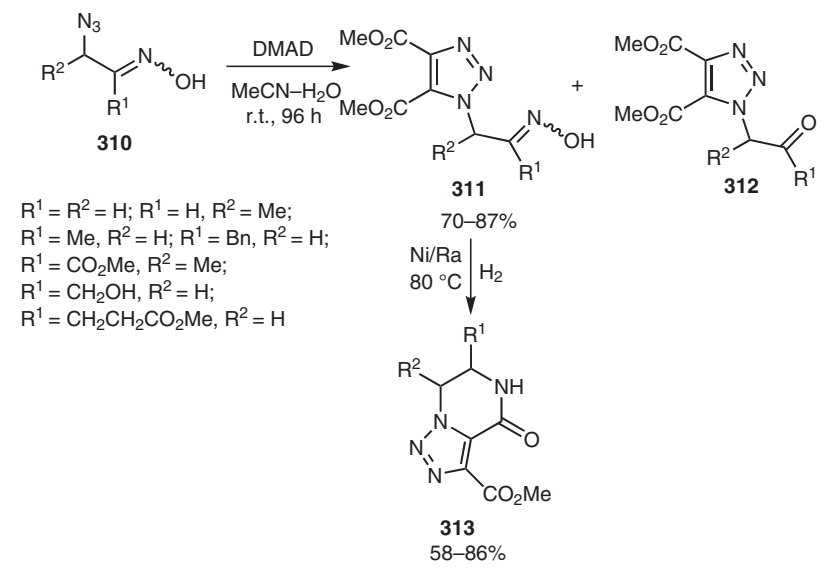

Scheme 86

\subsubsection{Nitriloxides}

Tandem electrophilic cyclization, [3+2] cycloaddition and rearrangement reactions of 2-alkynylbenzaldoximes $314\left(\mathrm{R}^{1}=\mathrm{H}, \mathrm{F} ; \mathrm{R}^{2}=\mathrm{Ph}, 4-\mathrm{MeOC}_{6} \mathrm{H}_{4}\right.$, etc. $)$, DMAD and bromine afforded the unexpected isoquinoline-based azomethine ylides 315 in good to excellent yields. The products could be further worked upon via palladiumcatalyzed cross-coupling reactions to generate highly functionalized isoquinoline-based stable azomethine ylides (Scheme 87). ${ }^{102}$

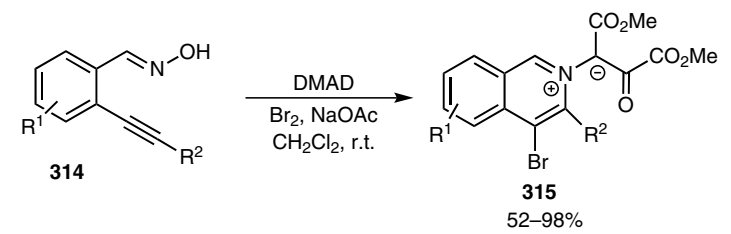

Scheme 87

\subsubsection{Diazoalkanes}

In the last ten years, the range of indazole derivatives with valuable biological activities increased substantially. Among them, agonists of estrogen receptors and dopamine $\mathrm{D}_{3}$ receptors, HIV protease inhibitors and new antiinflammatory substances have been discovered. Strakova et al. ${ }^{103}$ described the [3+2] cycloadditions of a series of tetrahydroindazoles $\mathbf{3 1 6}$ with DMAD that gave the spiro derivatives 317 (Scheme 88).

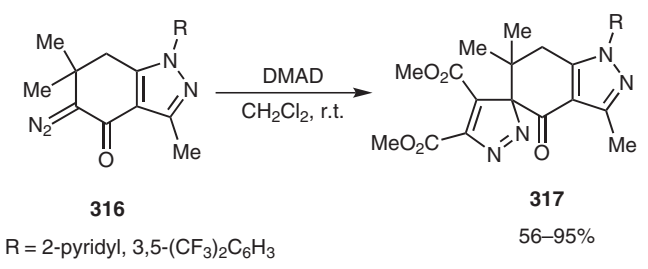

Scheme 88

\subsubsection{Sulfur Dipoles}

Indoles fused with the 1,2-dithiole-3-thione ring, as in compound 318, could be of interest because such compounds have a broad spectrum of biological activity and may be useful synthons for many sulfur heterocycles. ${ }^{104} \mathrm{~A}$ one-pot synthesis of derivatives $\mathbf{3 1 9}$ and $\mathbf{3 2 0}$ from $\mathbf{3 1 8}$ with DMAD by 1,3-dipolar cycloaddition was reported (Scheme 89). ${ }^{105,106}$

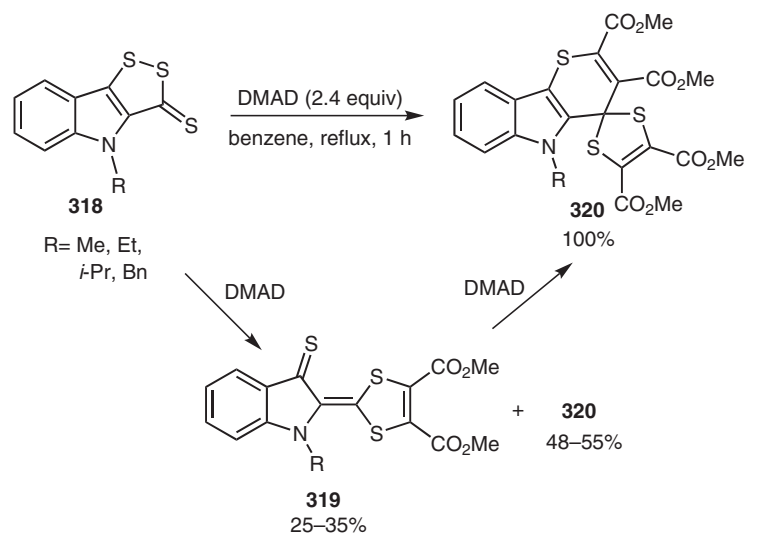

Scheme 89

Since the famous work by Wudl et al. on tetrathiafulvalene (TTF), ${ }^{107}$ long after dibenzotetrathiafulvalene was first reported, interest in this exceptional $\pi$-donor in the field of materials chemistry has been on a constant increase. DMAD, which is of particular interest due to its dienophilic and dipolarophilic properties, in combination with its electrophilicity, has been widely exploited in the development of highly functionalized tetrathiafulvalenes. A one-step synthesis of tetrathiafulvalenes $\mathbf{3 2 1}$ from carbon disulfide and DMAD, under high pressure (5000 atm) was proposed. ${ }^{108}$ Replacing carbon disulfide by carbon diselenide and carbon sulfide selenide allowed this reaction to be used to access analogues of tetrathiafulvalene (Scheme 90).

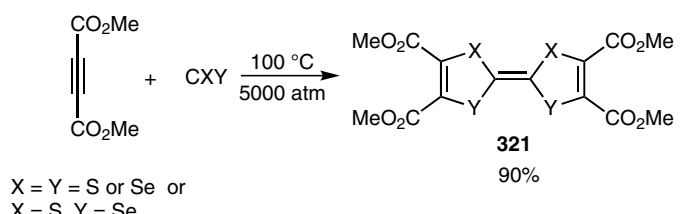

Scheme 90

2-Thioxo-1,3-dithioles 323, which are good precursors of tetrathiafulvalenes upon dimerization-desulfurization, can also be obtained from DMAD, carbon disulfide and either bis(2,2,6,6-tetramethylpiperidine) disulfide or bis(morpholino) disulfide $\mathbf{3 2 2}$ at $140{ }^{\circ} \mathrm{C}$ under nitrogen. ${ }^{109}$ This methodology is not restricted to electrophilic alkynes since it also works with terminal alkynes. A mechanism involving a 1,3-dipolar cycloaddition between carbon disulfide and a transient thioketocarbene was proposed (Scheme 91). 


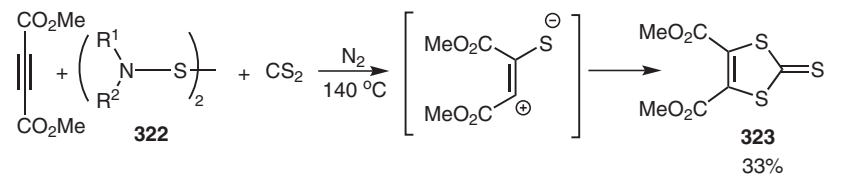

Scheme 91

In 1979, Cava and co-workers suggested a modification of Hartzler's procedure by adding a mixture of DMAD and tetrafluoroboric acid etherate, at $-65^{\circ} \mathrm{C}$, to tributylphosphine-carbon disulfide complex. The thus-formed ylide was protonated and then trapped as its phosphonium salt 326, which was isolated in $72 \%$ yield. ${ }^{110}$ This salt proved to be of high synthetic value in 1,3-dithiole and tetrathiafulvalene chemistry, affording compounds such as the derivatives 327 (Scheme 92). This general scheme was extended to the synthesis of diselenadithiafulvalene $\mathbf{3 3 0}$, the first step being a cycloaddition of DMAD to 2-thioxo1,3-diselenolane 328. It was also used for the synthesis of tetraselenafulvalene from ethylene triselenocarbonate (Scheme 93). ${ }^{111}$

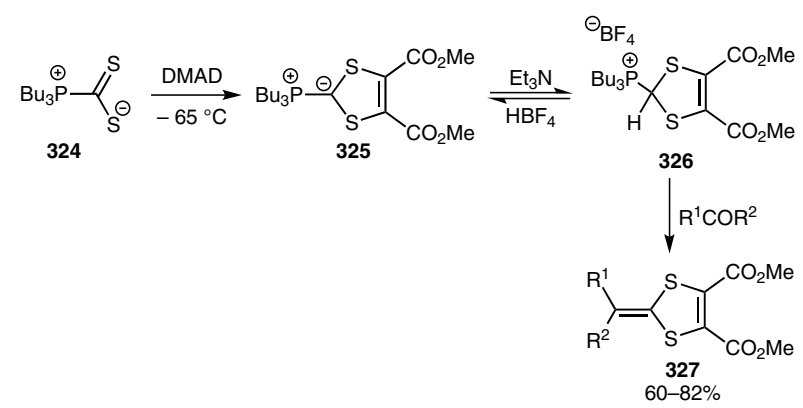

Scheme 92

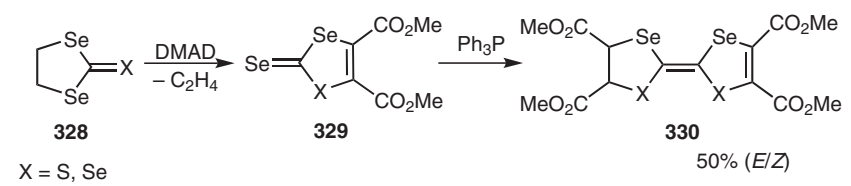

Scheme 93

\section{3 $[2+2]$ Cycloadditions}

Reactions of enamines of cyclic ketones with DMAD can be manipulated to achieve ring enlargement by a unit of two carbon atoms. The reactions proceed via [2+2] cycloaddition and subsequent ring opening of the intermediate cyclobutenes (Scheme 94). This becomes a valid strategy for constructing an extended $\pi$-conjugated system when applied to cyclic $\pi$-conjugated enamines. This method was also successfully used to achieve ring enlargements of thiophenes to thiepines, benzofurans to benzoxepines, indoles to benzazepines and pentalenes to azulenes. Dipyrrolidinyl-annulene $\mathbf{3 3 2}$ reacted with an excess of DMAD in refluxing toluene to give ring-enlarged annulene 333 (Scheme 95). ${ }^{112}$

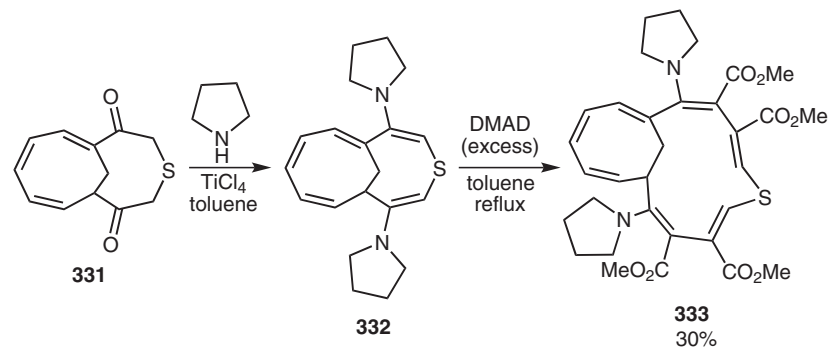

Scheme 95

Microwave-assisted regiospecific [2+2] cycloadditions of DMAD to derivatives 334, resulting in the formation of butadienes 335, were reported in 2008 (Scheme 96). ${ }^{113}$

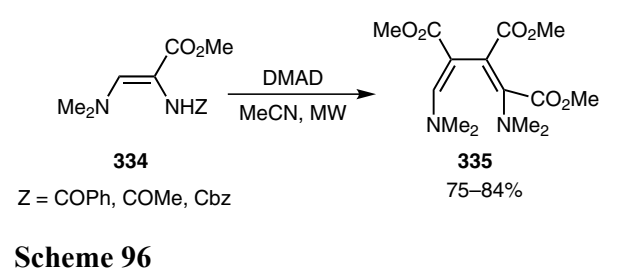

Moreover, the microwave-assisted [2+2] cycloaddition of DMAD to imidazolidine-2,4-dione $\mathbf{3 3 6 a}$, or to the corresponding thioxo derivative $\mathbf{3 3 6 b}$, in acetonitrile, produced the highly functionalized imidazolidine-2,4-dione derivatives 337 and 338 (Scheme 97). ${ }^{114}$

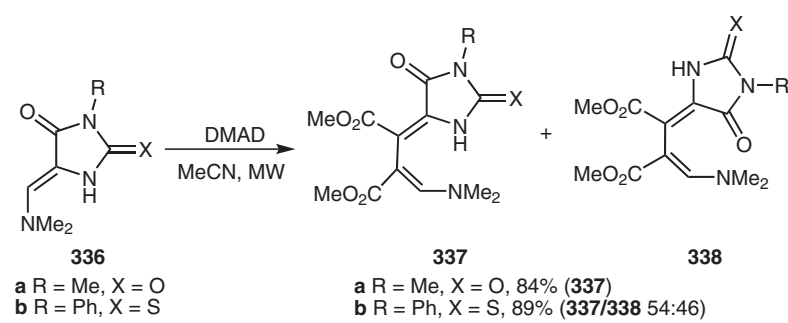

Scheme 97

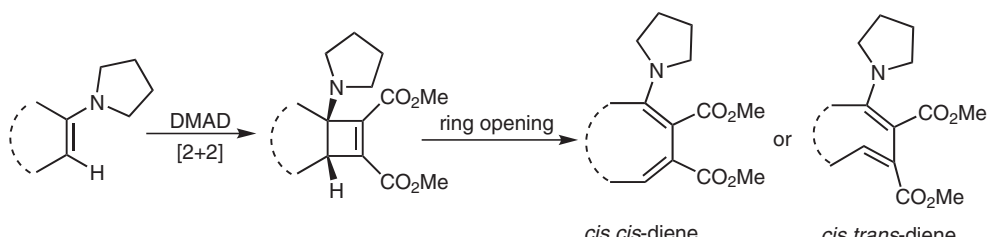

Scheme 94 
The reaction of the thiazole derivatives $\mathbf{3 3 9}$ with DMAD led unexpectedly to compounds 341 , which resulted from a sequence of reactions initiated by a [2+2] cycloaddition of DMAD to the formal carbon-carbon double bond of the thiazole ring (Scheme 98). ${ }^{115}$

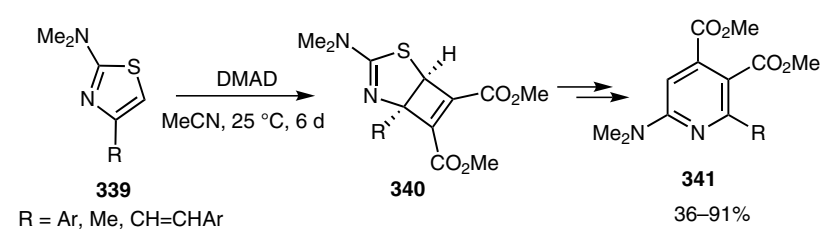

Scheme 98

Sajna et al. ${ }^{66}$ described the reaction of allenylphosphonate 342 with DMAD under neat conditions to afford compounds 343,344 and $\mathbf{3 4 5}$ following a plausible [2+2] cycloaddition (Scheme 99).

\section{$3.4 \quad[8+2]$ Cycloadditions}

Orbital-symmetry-allowed [8+2] cycloadditions between tetraenes 346 and tetraenophiles 348 , can in theory provide a straightforward approach for the synthesis of 10membered-ring compounds. Before the year 2003, however, all of the reported [8+2] cycloadditions were limited to geometrically constrained tetraenes (such as heptafulvenes, tropones, and indolizines), in which the terminal carbons or heteroatoms at positions 1 and 8 were rigidly held in close proximity. ${ }^{116}$

Kuznetsov et al. ${ }^{117}$ reported that although 5-bromoindolizine $\mathbf{3 5 0}$ was found to be passive toward nucleophiles, its reaction with DMAD led to cycl[3.2.2] azine 351 in $87 \%$ yield, through an [8+2] cycloaddition (with $\mathrm{HBr}$ elimination). This $[8+2]$ cycloaddition of dienophiles, across positions 3 and 5 , is a well-known type of indolizine reactivity (Scheme 100).

In 2003, it was reported that dienylisobenzofurans $\mathbf{3 5 2}$ could react with DMAD to furnish, as the major products, [8+2] adducts possessing the 11-oxabicyclo[6.2.1] undecane ring system 353. ${ }^{118 a}$ It was later noted that dienylfurans 354 could also participate in [8+2] cycloadditions with DMAD, affording compounds 355 (Scheme 101). ${ }^{118 b}$

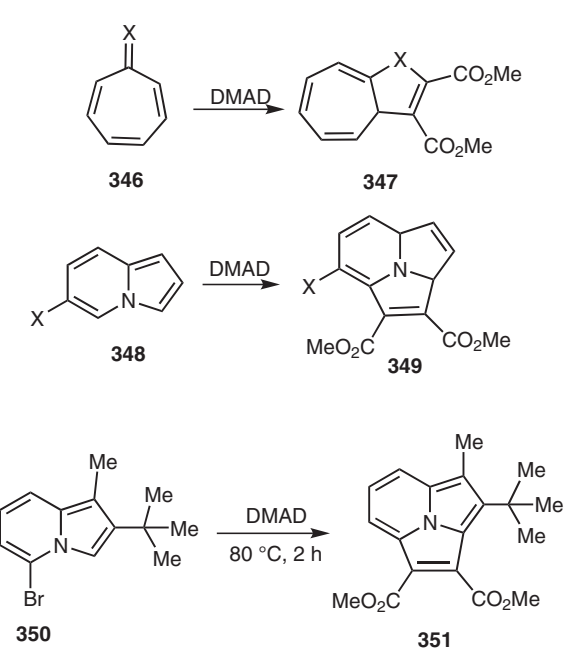

Scheme 100<smiles>[R]C=C([R])C(=C[10CH3])c1oc([13CH3])c2ccccc12</smiles>

$\mathrm{R}^{1}=\mathrm{H}, \mathrm{Me}, \mathrm{Bu}, \mathrm{TMS}$

$\mathrm{R}^{2}-\mathrm{R}^{3}=-\left(\mathrm{CH}_{2}\right)_{3^{-}},-\mathrm{O}\left(\mathrm{CH}_{2}\right)_{3}, \mathrm{Me}-\mathrm{H}$

$\mathrm{R}^{4}=\mathrm{Ph}, 2$-benzofuryl
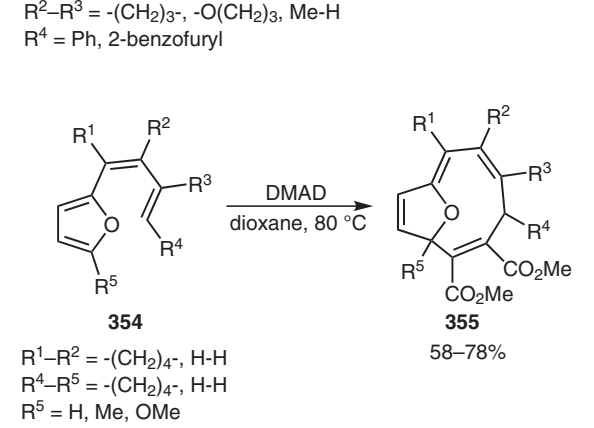

Scheme 101

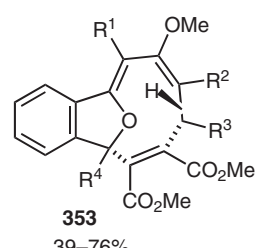

These [8+2] cycloadditions provided a direct approach for the synthesis of ring skeletons such as eleutherobin, briarellins and other natural products that have anticancer activity. Ten-membered-ring compounds with an oxygen bridge can also be readily synthesized through these $[8+2]$ cycloadditions.

Roy and Ghorai ${ }^{119}$ reported a one-pot three-component coupling of $o$-alkynylheteroaryl carbonyl derivatives 356

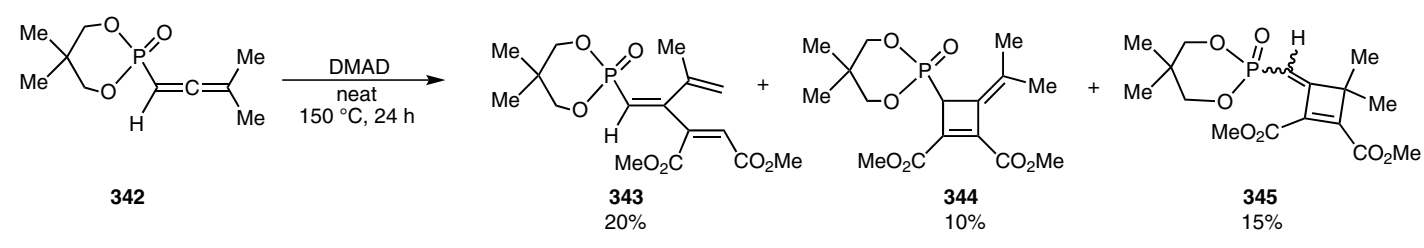

Scheme 99 
with $\alpha, \beta$-unsaturated Fischer carbene complexes and DMAD, leading to the synthesis of heterocyclic analogues of furanophane derivatives $\mathbf{3 5 8}$ through an [8+2] cycloaddition reaction (Scheme 102).

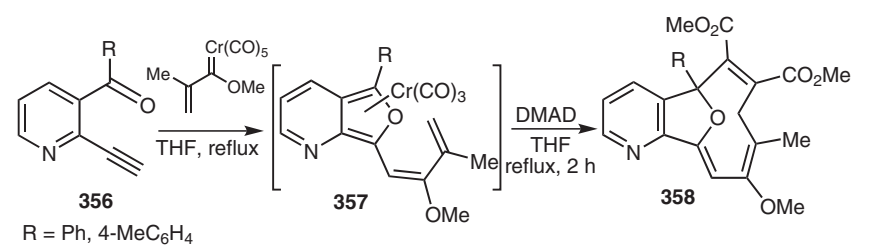

Scheme 102

\section{DMAD and the Generation of Zwitterions; Multicomponent Reactions (MCRs)}

Zwitterions are transient intermediates formed by the addition of neutral nucleophiles to electrophilic receptors. Zwitterions are a unique tool in organic synthesis leading to a variety of heterocycles. These reactive intermediates can be captured by suitable substrates (e.g., nucleophiles), after a series of transformations. In fact, it was found that in the case of acetylenedicarboxylate, three reaction paths are possible (paths A, B and C). Basically, in both paths A and $\mathrm{C}$ the nucleophile is added irreversibly, leading to a multi-component reaction. On the other hand, in path $\mathrm{B}$ the nucleophile gets eliminated from the system, and thus plays a catalytic role, conducting a two-component reaction. Path B usually involves nucleophiles, such as phosphines and tertiary amines such as pyridines and quinolines, while paths $\mathrm{A}$ and $\mathrm{C}$ involve mainly nucleophilic heterocyclic carbenes (NHCs) and isocyanides (Scheme 103).

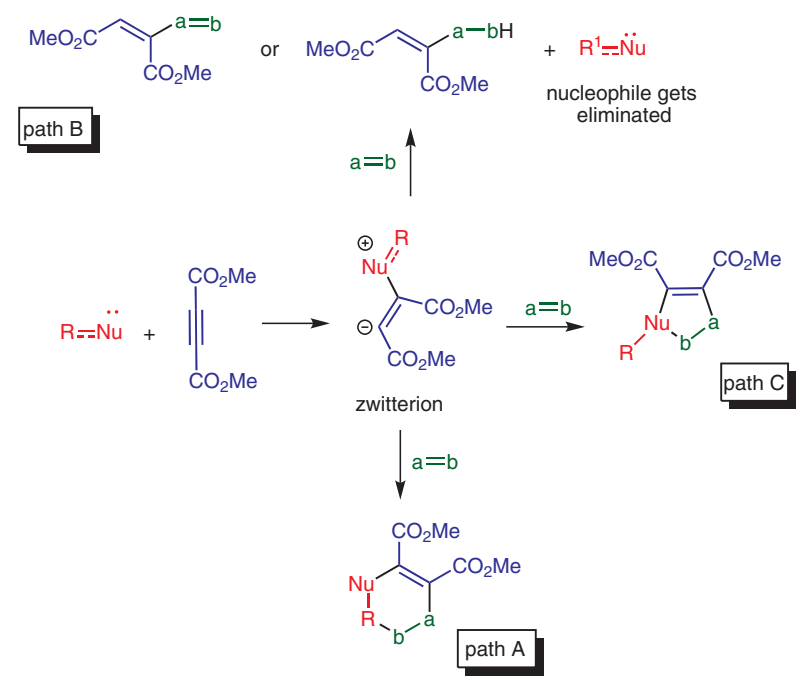

Scheme 103

\subsection{Phosphines and Derivatives}

The importance of organophosphoric compounds as reagents in organic synthesis and as transition-metal-cata- lyst ligands has been very actively studied and proven in organic laboratories in recent years. Particular attention has been paid, by synthetic researchers, to both the properties and the reactive behavior of phosphorus ylides in a multitude of applications in natural product synthesis, which is of course vital in biomedicinal chemistry and pharmaceutical design. Phosphorus ylides, endowed by unique electronic and molecular structures, are classed as special zwitterions, useful in diverse reactions. They are characterized by electron-rich carbanions, decisively nucleophilic; thus availing themselves to deployment as starting reagents in organic synthesis projects. Most importantly, phosphorus ylides are readily obtainable from abundantly available inexpensive reagents and have been correspondingly researched in depth with respect to their reactive properties and their potential in both reagent preparation and industrial-level organic synthesis. Ylide preparation usually involves the treatment of a phosphonium salt (normally from phosphine and an alkyl halide) with a base. Phosphines and DMAD in the presence of organic acids could also be used for the preparation of ylides (Scheme 104). ${ }^{120}$

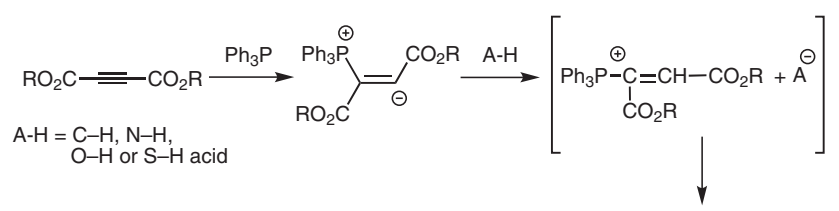

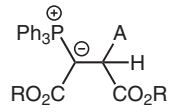

Scheme 104

\subsubsection{Reactions of Triphenylphosphine, DMAD and C-H Acids}

There are many studies on the reaction between trivalent phosphorus nucleophiles and acetylenic esters in the presence of $\mathrm{C}-\mathrm{H}$ acids. In some cases the ylide products are stable, but in other cases they cannot be isolated and appear to occur as intermediates on the pathway to an (eventually) observed product.

Stabilized phosphorus ylides have also been isolated from the related reactions of triphenylphosphine, DMAD and acyclic and cyclic 1,3-diketones. The reaction of DMAD with keto-nitriles $\mathbf{3 6 0}$ in the presence of triphenylphosphine (359) led to the stabilized phosphorus ylides 361 (Scheme 105). ${ }^{121}$

The reaction of trifluoro diones 362 with DMAD, in the presence of triphenylphosphine, provided a simple onepot reaction for the synthesis of polyfunctionalized trifluoromethylated cyclobutene derivatives 363 via an intramolecular Wittig reaction in high yield (Scheme 105). ${ }^{122}$

2-Acetylbutyrolactone (364) underwent a smooth reaction with triphenylphosphine and DMAD to produce stabilized diastereomeric phosphorus ylides 365 possesing two stereogenic centers. These compounds underwent, in boil- 


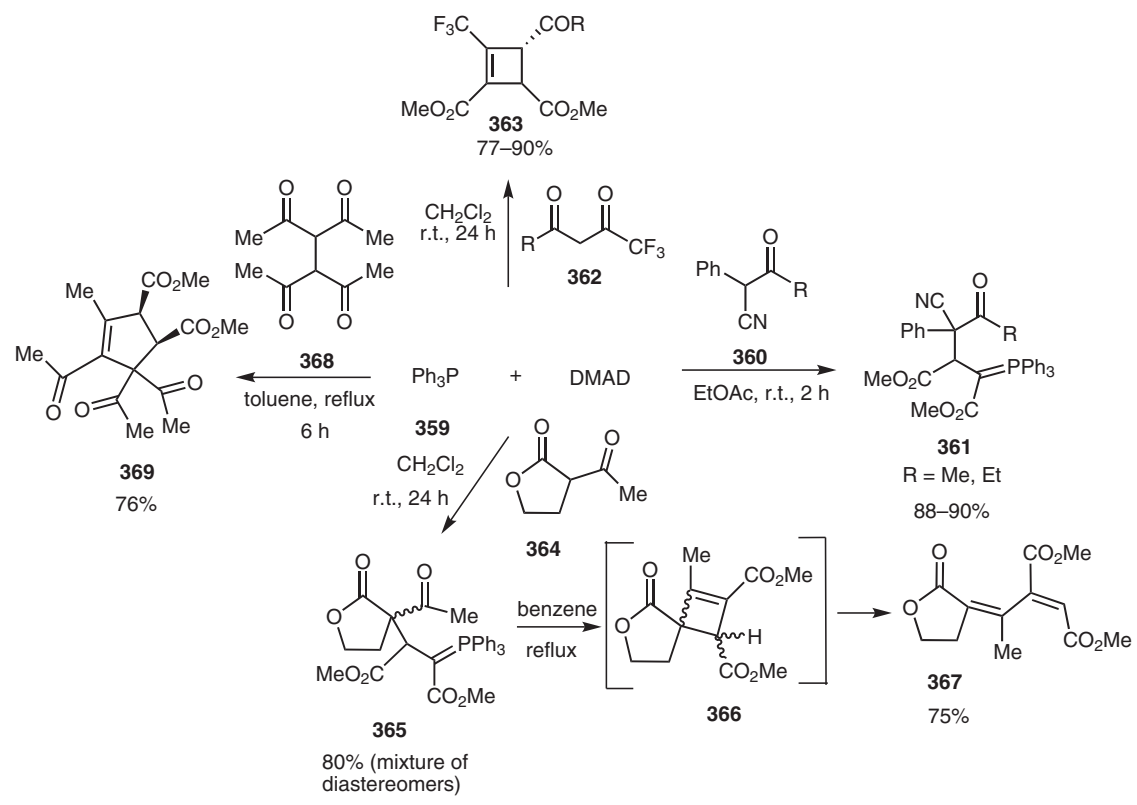

Scheme 105

ing benzene, an intramolecular Wittig reaction to produce highly strained spiro compounds $\mathbf{3 6 6}$ which spontaneously underwent ring-opening to produce functionalized 1,3dienes 367 (Scheme 105). ${ }^{123}$

On the other hand, 3,4-diacetylhexane-2,5-dione (368) in its reaction with DMAD, in the presence of triphenylphosphine, underwent a diastereoselective intramolecular Wittig reaction to produce the cyclopentene derivative 369 in good yield (Scheme 105). ${ }^{124}$

\subsubsection{Reactions of Triphenylphosphine, DMAD and N-H Acids}

Reaction of the reactive 1:1 intermediate adduct of triphenylphosphine and DMAD with 3-chloroindole-2-carbaldehyde $(\mathbf{3 7 0})^{125}$ led to a vinylphosphonium salt which underwent an intramolecular Wittig reaction to produce the corresponding pyrrole derivative 372 (Scheme 106). Moreover, the reaction of the 1:1 triphenylphosphineDMAD adduct with some other $\mathrm{N}-\mathrm{H}$ acids delivered the corresponding pyrrole derivatives via an intramolecular Wittig reaction.

The reaction of arylsulfonamide derivatives of 2-aminobenzaldehyde 373, DMAD and triphenylphosphine produced dihydroquinoline derivatives $\mathbf{3 7 5}$ in excellent yields (Scheme 106). ${ }^{126}$

In addition, the DMAD-triphenylphosphine intermediate was trapped by ethyl $1 H$-pyrimidine-2-carboxylate 376 to the pyrimidine derivatives $\mathbf{3 7 7}$ and $\mathbf{3 7 8}$ in a nearly 7:1 ratio and overall good yields (Scheme 106). ${ }^{127}$
A series of triazene derivatives $\mathbf{3 8 0}$ with polyfunctional substituents, such as the ylide moiety and ester groups, were synthesized by the reaction of DMAD with 1,3-diaryl-1-triazenes $\mathbf{3 7 9}$ in the presence of triphenylphosphine in ethyl acetate (Scheme 106). ${ }^{128}$

A convenient synthesis of highly functionalized phosphorus ylides was achieved by the reaction of DMAD with $N$ phenylacetamides 381 in the presence of triphenylphosphine. The intramolecular cyclization of ylide 382 in toluene, at reflux, gave the pyrazole derivative $\mathbf{3 8 3}$ in a high yield (Scheme 106). ${ }^{129}$

\subsubsection{Reactions of Triphenylphosphine, DMAD and O-H Acids}

Johnson and Tebby ${ }^{130}$ established the intermediacy of a zwitterion in the reaction of triphenylphosphine with DMAD, which proved to form the basis of a wide variety of later transformations. These zwitterionic species were shown to undergo annulation reactions with electrophiles, such as aldehydes, $\alpha$-keto nitriles, $\alpha$-keto esters and $N$-tosylimines, to provide highly substituted unsaturated $\gamma$-lactones and lactams. ${ }^{131}$

Recently, the reaction between DMAD and various aryl aldehydes in the presence of triphenylphosphine was reported, leading to unsaturated $\gamma$-butyrolactone derivatives 385 and highly substituted enones $\mathbf{3 8 6}$ in fairly good yields at room temperature (Scheme 107). ${ }^{132}$ 


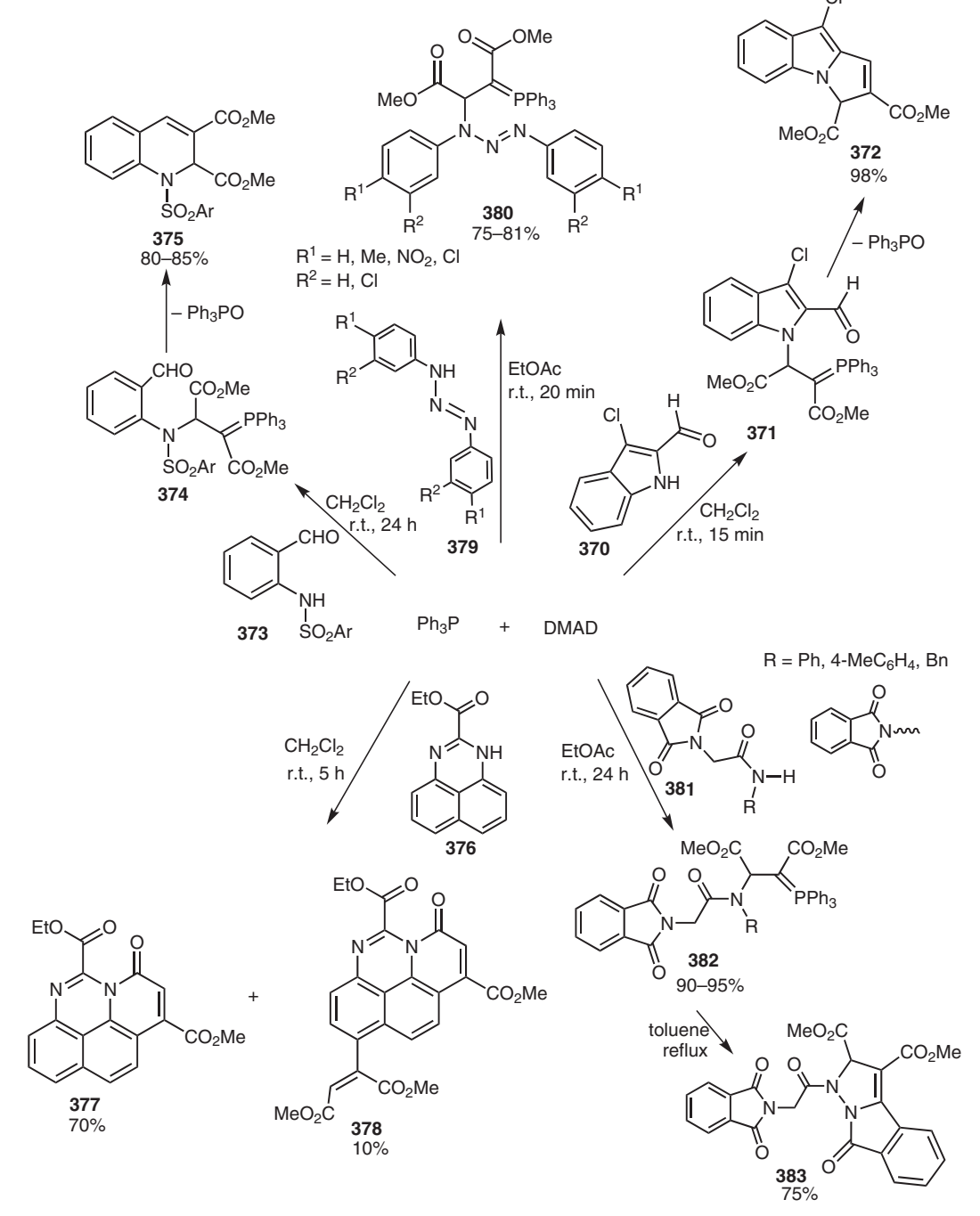

Scheme 106

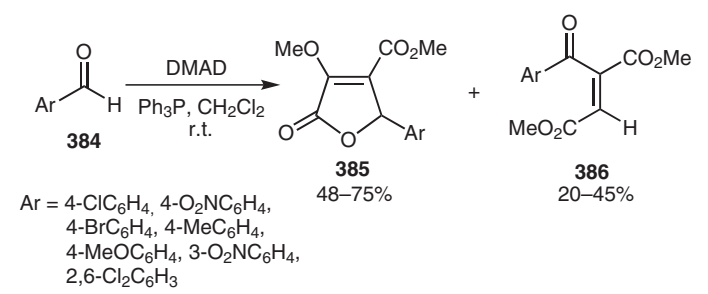

Scheme 107

The reaction between arylglyoxal monohydrates $\mathbf{3 8 7}$, DMAD and triphenylphosphine led to the dihydrofuran derivatives $\mathbf{3 8 9}$ by a simple and efficient method (Scheme 108). ${ }^{133}$

The one-pot reaction between ninhydrin 391, amide derivatives 390, DMAD and triphenylphosphine led to oxacyclopenta $[a]$ indene derivatives 392 (Scheme 109). ${ }^{134}$

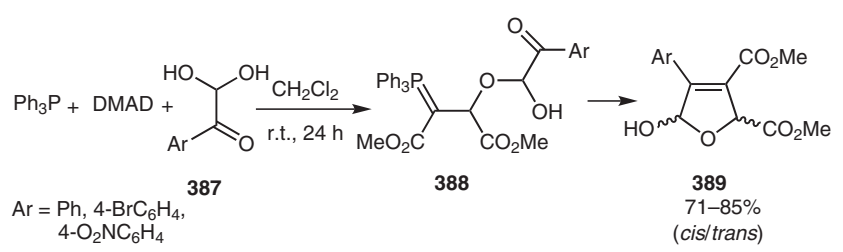

\section{Scheme 108}

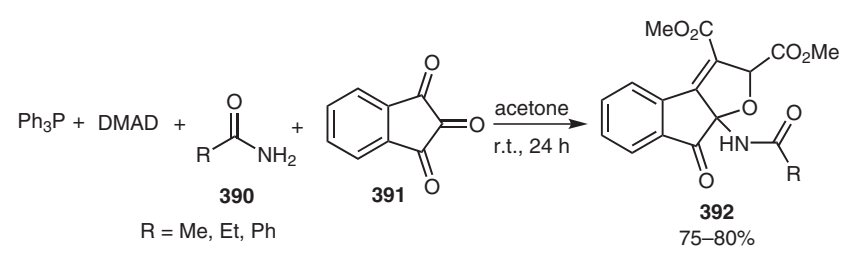

Scheme 109 
Yavari et al. ${ }^{135}$ described a new and operationally convenient approach to the synthesis of 4-carboxymethylcoumarins based on the aromatic electrophilic substitution between the conjugate base of a substituted phenol and a vinyltriphenylphosphonium salt derived from the reaction of DMAD with triphenylphosphine. In this context, 4-carboxyalkyl-8-formyl coumarins 394 were synthesized from 2-hydroxybenzaldehydes $\mathbf{3 9 3}$ in good yields via vinyltriphenylphosphonium salt mediated electrophilic aromatic substitution. The salt was generated in situ by protonation of the reactive 1:1 intermediate produced by the reaction of triphenylphosphine and DMAD with the 2hydroxybenzaldehyde (Scheme 110). ${ }^{136}$

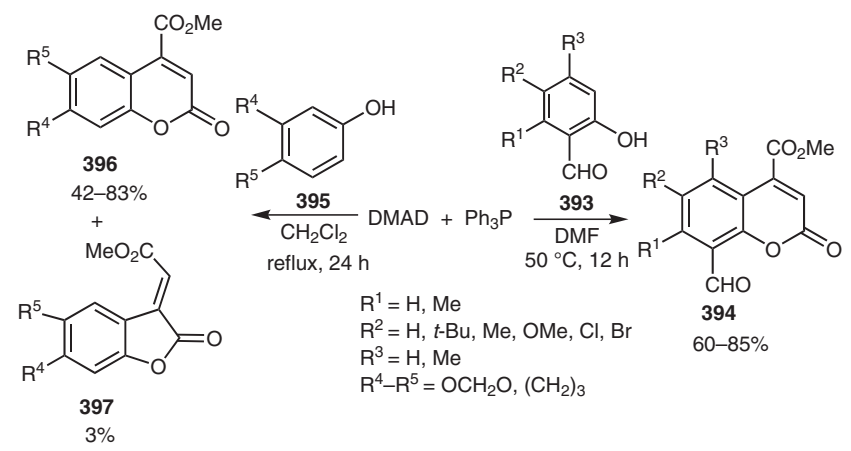

Scheme 110

Moreover, Symeonidis et al. ${ }^{137}$ reported the synthesis of linear [6,7]-fused coumarins 396, along with a minor product 397, which were obtained from the reaction of $[3,4]$-fused phenols 395 with DMAD and triphenylphosphine (Scheme 110). The synthesis of angular [7,8]-fused coumarins from the reaction of [2,3]-fused phenols with DMAD and triphenylphosphine was also reported. ${ }^{137}$
Novel spirocyclic lactones 399 and 401 were synthesized by Nair et al. ${ }^{131}$ who carried out a phosphine-mediated reaction of DMAD with $o$ - and $p$-quinones 398 and $\mathbf{4 0 0}$. The zwitterion, not surprisingly, exhibited a complete preference for the quinone carbonyl group, leaving the enone double bond intact (Scheme 111).

\subsubsection{Reactions of Triphenylphosphine, DMAD and S-H Acids}

Stable crystalline phosphorus ylides $\mathbf{4 0 3}$ were obtained in excellent yields from the 1:1:1 addition reaction between triphenylphosphine and DMAD in the presence of 1-methylimidazole-2-thiol (402). These sulfur-containing phosphoranes 403 existed in solution as a mixture of two geometrical isomers, owing to restricted rotation around the carbon-carbon double bond resulting from conjugation of the ylide unit with the adjacent carbonyl group (Scheme 112). ${ }^{138}$

Analogously, the reaction of triphenylphosphine and DMAD in the presence of thiophenol derivatives 404 proceeded spontaneously, at room temperature, to produce stable phosphorus ylides 405 (Scheme 112). ${ }^{139}$ The reaction of some other strong $\mathrm{S}-\mathrm{H}$ acids with DMAD, in the presence of triphenylphosphine, led to the corresponding phosphoranes. ${ }^{140}$

Crystalline phosphorus ylides $\mathbf{4 0 7}$ were obtained in excellent yields from the 1:1:1 addition reaction between triphenylphosphine, DMAD and $\mathrm{N}-\mathrm{H}$ or $\mathrm{S}-\mathrm{H}$ acids 406 such as 2-amino-4-phenylthiazole, 2-amino-5-(3-chlorobenzyl)thiadiazole, 3-amino-2-methylquinazolin-4-one and 3-amino-2-mercaptoquinazolin-4-one (Scheme 113). ${ }^{141}$

Scheme 111
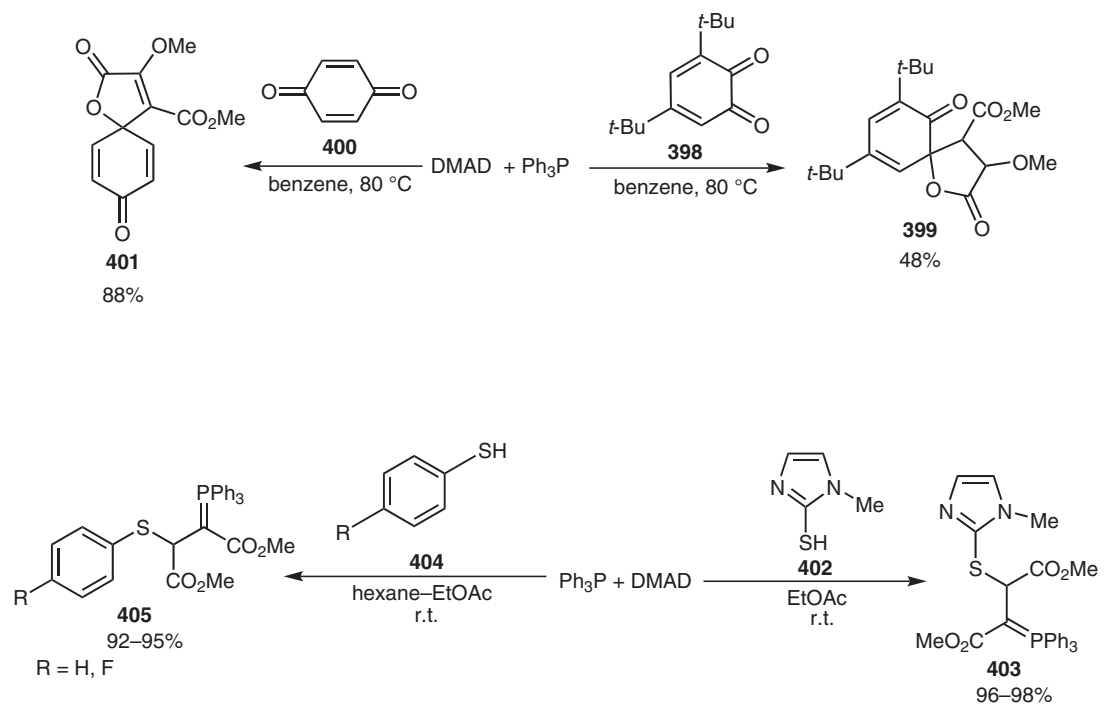

Scheme 112 
<smiles>Nn1c(S)nc2ccccc2c1=O</smiles>

406

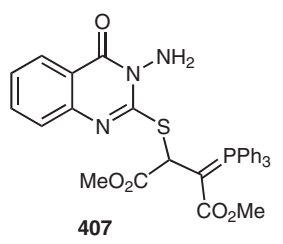

$87 \%$

Scheme 113

\subsubsection{Reactions of Triphenylphosphine, DMAD and Oxa- or Azadienes}

Waldmann and co-workers, ${ }^{142}$ wishing to synthesize compound collections inspired by natural products for chemical biology and medicinal chemistry research, developed an enantioselective triphenylphosphine- or tributylphosphine-organocatalyzed asymmetric [4+2] annulation between electron-deficient heterodienes and acetylene derivatives. By using 3-formylchromones 408, DMAD and triphenylphospine (up to $30 \mathrm{~mol} \%$ ), tricyclic benzopyrones $409(\mathrm{X}=\mathrm{O})$ were isolated in high yields; these were further used for an efficient synthesis of tetrahydroindolo[2,3- $a$ ]quinolizidines (centrocountins). Analogously, from the reaction of 3-formylchromone $N$-tosylimines $408(X=N T s)$ the tricyclic products $409(X=N T s)$ along with the hydroxybenzoylpyridines $\mathbf{4 1 0}$ were formed. Finally, the reaction of acyclic electron-poor oxadienes $\mathbf{4 1 1}$ with DMAD and triphenylphosphine was also studied, whereupon the initially detected [4+2]-annulation products underwent a subsequent Claisen rearrangement to yield dehydropyrans 412 (Scheme 114).

\subsubsection{Reactions of Phosphine Derivatives with DMAD}

The nucleophilic addition of trialkyl phosphites to electron-deficient triple bonds led to highly reactive zwitterionic intermediates, which could be trapped by various electrophiles. Phosphonate esters are an important class of compounds obtained by the sequential addition reaction of trivalent phosphite with $\alpha, \beta$-unsaturated carbonyl molecules in the presence of $\mathrm{C}-\mathrm{H}$ or $\mathrm{N}-\mathrm{H}$ acids. Synthesis of phosphonato esters 414 and $\mathbf{4 1 5}$ was accomplished via reaction between DMAD and triphenyl phosphite in the presence of biological compounds $\mathbf{4 1 3}$ such as theophylline, 4-hydroxypyrimidine, $2 H$-3,1-benzoxazine-2,4(1H)dione, 2-chloroaniline or 3-nitroaniline at ambient temperature (Scheme 115). ${ }^{143,144}$

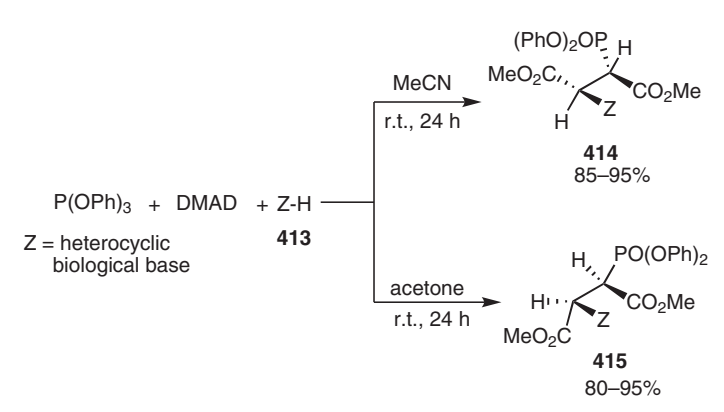

Scheme 115

Adib et al. ${ }^{145}$ described an efficient and chemoselective sulfonamide N-alkylation of sulfonyl ureas. The sulfonyl urea derivatives, prepared in situ by the addition of an ar-
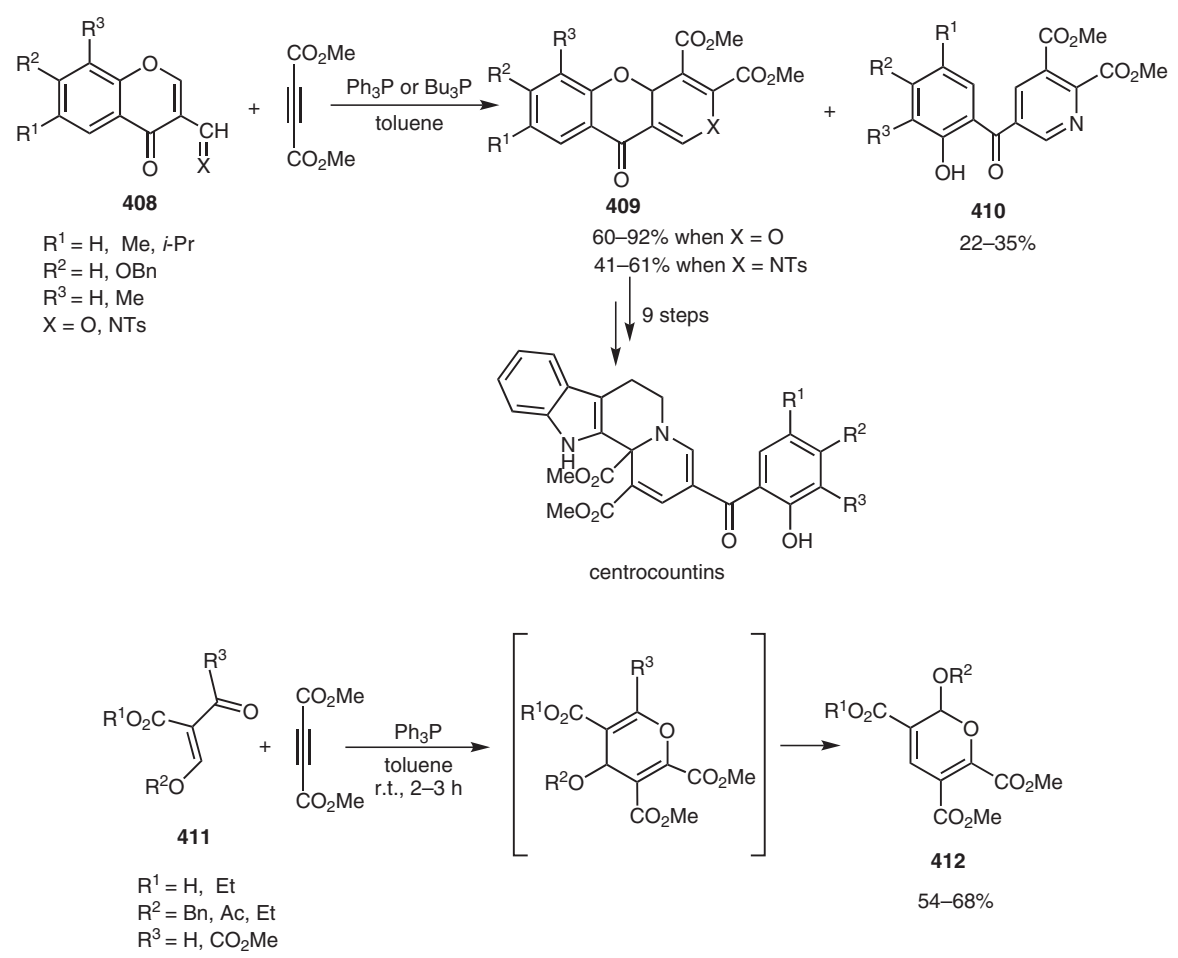

Scheme 114 


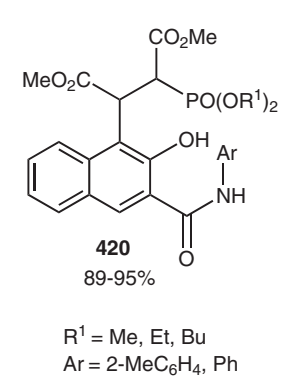

$\mathrm{R}^{1}=\mathrm{Me}, \mathrm{Et}, \mathrm{Bu}$ Ar $=2 \mathrm{MeC}_{6} \mathrm{H}_{4}, \mathrm{Ph}$

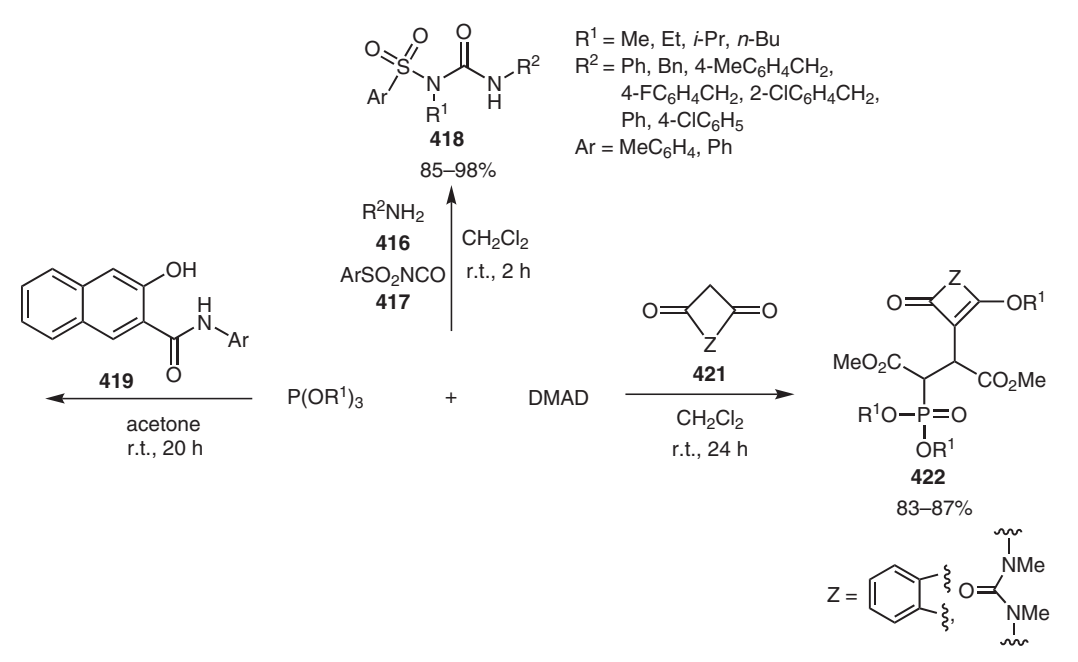

\section{Scheme 116}

omatic amine $\mathbf{4 1 6}$ to an arylsulfonyl isocyanate 417 , were selectively alkylated to give $\mathbf{4 1 8}$ in excellent yields under neutral and mild conditions by treatment with trialkyl phosphite-DMAD at ambient temperature (Scheme 116).

A three-component reaction between DMAD and trialkyl phosphites in the presence of $N$-aryl-3-hydroxynaphthalene-2-carboxamide 419 led to dialkyl 2-(dimethoxyphosphoryl)-3-[2-hydroxy-3-(arylcarbamoyl)naphthalen-1-yl] succinates 420 in excellent yields (Scheme 116). ${ }^{146}$

Protonation of the reactive intermediate produced in the reaction between trialkyl(aryl) phosphites and DMAD by $\mathrm{C}-\mathrm{H}$ acids 421 such as indane-1,3-dione and $N, N^{\prime}$-dimethylbarbituric acid, led to functionalized phosphonates $\mathbf{4 2 2}$ in good yields (Scheme 116). ${ }^{146}$

Deng et al. ${ }^{147}$ reported the synthesis of aryl-substituted $\gamma$ lactones 425 bearing an $\alpha$-phosphorus ylide moiety, in moderate to good yields, through the assembly of DMAD, electron-deficient aldehydes $\mathbf{4 2 3}$, and triaryl- or trialkylphosphanes (Scheme 117).

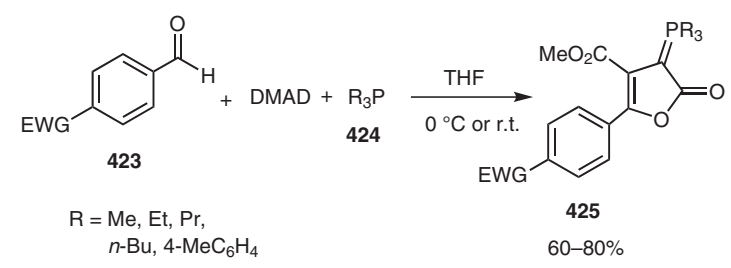

Scheme 117

A new class of phosphorus-ylide containing conjugate heterocycles was isolated from a mixture of colored products of the reaction of a silylphosphine $\mathbf{4 2 6}$ and DMAD. The indigo-like bis-phosphole structure 427 appeared with a green to blue color because of the low energy gap of the phosphole (Scheme 118). ${ }^{148}$

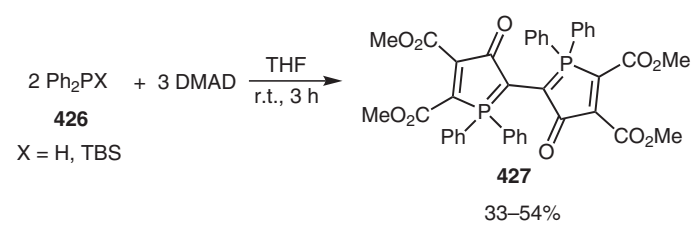

Scheme 118

The reaction of dihydrophosphete $\mathbf{4 2 8}$ with an excess of DMAD was found to give the ring-expanded product $\mathbf{4 2 9}$ (Scheme 119), ${ }^{149 a}$ fully characterized by X-ray crystal structure analysis. A similar rearrangement was proposed in the case of 1-phenyl-3,4-dimethylphosphole $(\mathbf{4 3 0})^{149 \mathrm{~b}}$ yielding the ring-expanded product $\mathbf{4 3 1}$. However, fairly recently Duan et al. ${ }^{149 \mathrm{c}}$ reinvestigated the reaction and established the formation of the stable ylide $\mathbf{4 3 2}$.

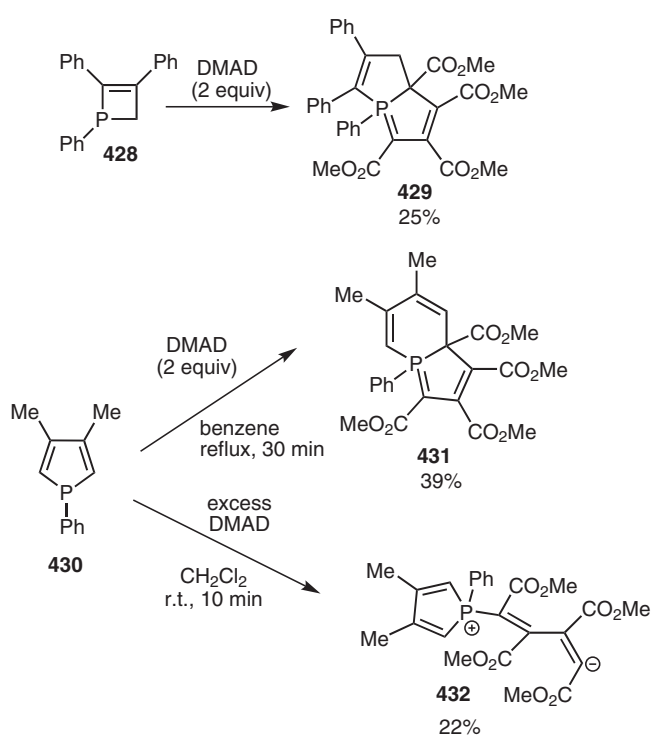

Scheme 119 


\subsection{Amines}

\subsubsection{Reactions of Primary Aromatic or Aliphatic Amines}

Although not quite as productive as phosphine catalysis, amines can also initiate various transformations by generating zwitterionic intermediates from activated olefins and acetylenes.

A protocol has been developed for the efficient synthesis of structurally diverse 3,4-dihydropyridin-2(1H)-ones 436 and 3,4-dihydro- $2 H$-pyrans $\mathbf{4 3 8}$ via four-component reactions of arylamines $\mathbf{4 3 3}$, DMAD, aromatic aldehydes 434 and cyclic 1,3-diketones $\mathbf{4 3 5}$ and $\mathbf{4 3 7}$, respectively. The selective formation of the very different pyridinone or pyran derivatives was found to depend on the structure of the cyclic 1,3-diketone (Scheme 120). ${ }^{150}$

In addition, a practical and efficient procedure for the preparation of the polysubstituted dihydropyridines $\mathbf{4 4 0}$ was developed through a unique four-component reaction of aromatic aldehydes, nitriles 439, arylamines and DMAD (Scheme 120). ${ }^{151}$

A facile and efficient synthesis of tetrasubstituted 1,4- and 1,6-dihydropyridines $\mathbf{4 4 3}$ and $\mathbf{4 4 4}$ was achieved by employing a three-component domino reaction using DMAD, aliphatic amines 441 and $\alpha, \beta$-unsaturated aldehydes $\mathbf{4 4 2}$ in the presence of trifluoroacetic acid. Interestingly, regioselectivity in the synthesis of 1,4dihydropyridines was increased by using triflic acid (Scheme 121). ${ }^{152}$
The 1,4-dihydropyridines $\mathbf{4 4 3}$ were further used for the synthesis of fused-naphthyridine derivatives $\mathbf{4 4 7}$ and $\mathbf{4 4 8}$ by reaction with aromatic amines $\mathbf{4 4 5}$ and aromatic aldehydes $\mathbf{4 4 6}$ catalyzed by boron trifluoride-diethyl etherate in acetonitrile (Scheme 122). ${ }^{152}$

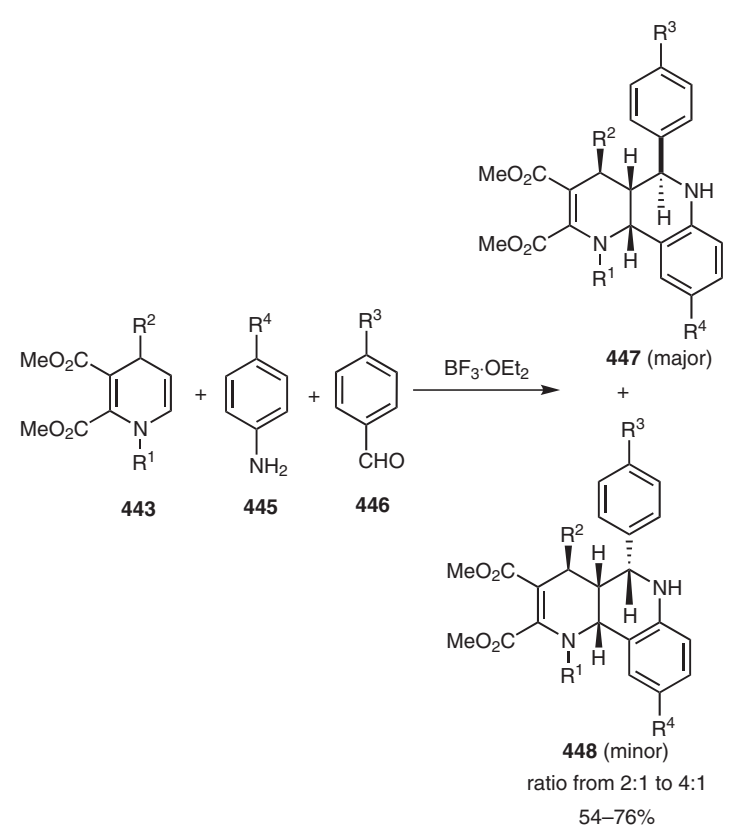

Scheme 122

Polyethylene glycol (450) and iron(III) chloride were found to be an inexpensive, non-toxic and effective medi-

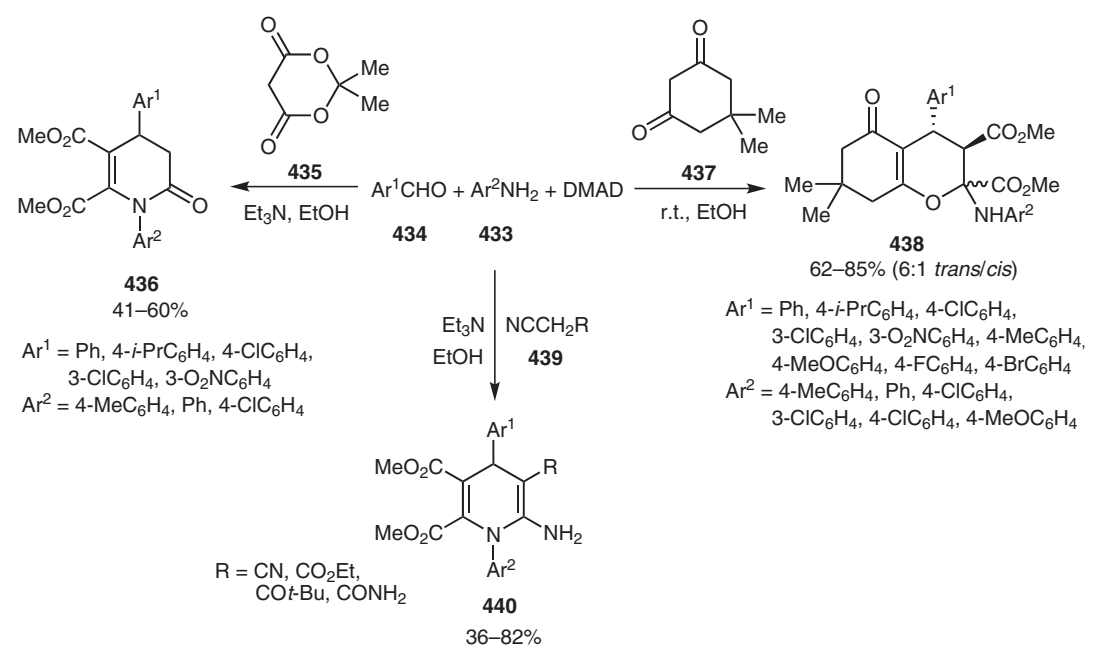

Scheme 120

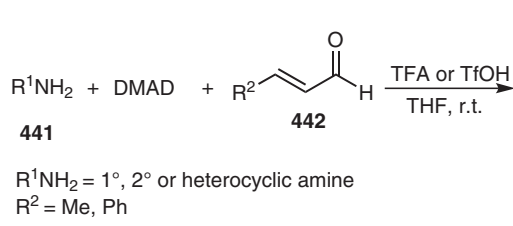

$\mathrm{R}^{2}=\mathrm{Me}, \mathrm{Ph}$

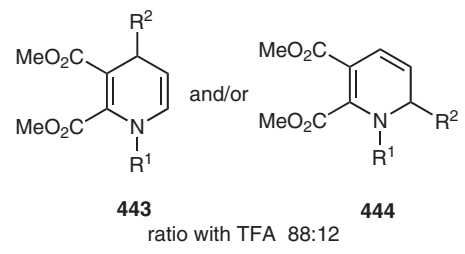

$56-83 \%$

Scheme 121 
um and catalyst, respectively, for the one-pot synthesis of highly functionalized pyrroles 451 using bromides 449 , amines and DMAD. Utilizing this protocol, various pyrrole derivatives were synthesized in excellent yields. Environmental acceptability, low cost, high yields and the recyclability of the polyethylene glycol are the important features of the protocol (Scheme 123). ${ }^{153 a, b}$

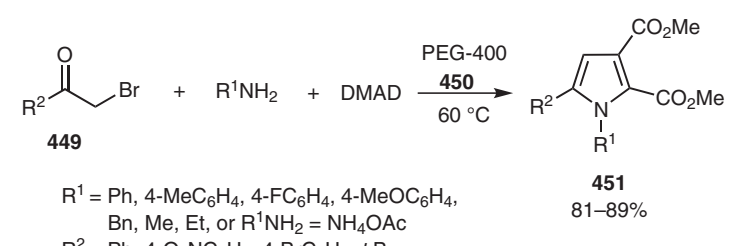
$\mathrm{R}^{2}=\mathrm{Ph}, 4-\mathrm{O}_{2} \mathrm{NC}_{6} \mathrm{H}_{4}, 4-\mathrm{BrC}_{6} \mathrm{H}_{4}, t-\mathrm{Bu}$

\section{Scheme 123}

The four-component reaction of DMAD, aromatic aldehydes 452, benzylamines 453 and malononitrile (454) led to polyfunctionalized 1,4-dihydropyridine derivatives $\mathbf{4 5 5}$ (Scheme 124). ${ }^{154}$

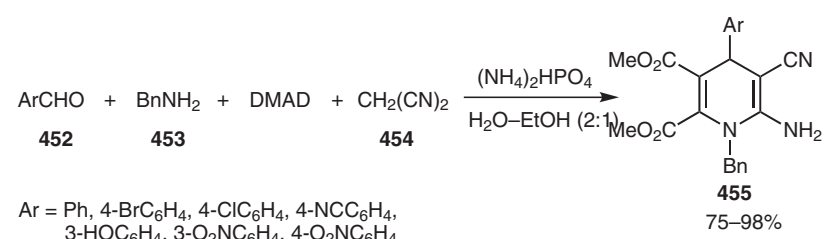

Scheme 124

\subsubsection{Reactions of Tertiary Amines}

Stereoselective reaction of various substituted phenols 456 with DMAD, in the presence of a catalytic amount of an aqueous solution of a trialkylamine $\mathbf{4 5 7}$ in dichloromethane, led to dimethyl 2-phenoxymaleates $\mathbf{4 5 8}$ in good to excellent yields under mild conditions (Scheme 125). ${ }^{155}$

The 1,4-dipole derived from 4,5-dimethylthiazole (460) and DMAD was shown to react readily with chromone-3carboxaldehydes 459, resulting, after an unusual rear-

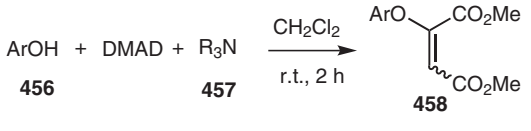

$$
\begin{aligned}
& 84-96 \%
\end{aligned}
$$

\section{Scheme 125}

rangement, in the facile synthesis of thiazolo[3,2- $a$ ]pyridine derivatives 462 . In some instances, tetracyclic chromenothiazolopyridines $\mathbf{4 6 1}$ were formed (Scheme 126). ${ }^{156}$

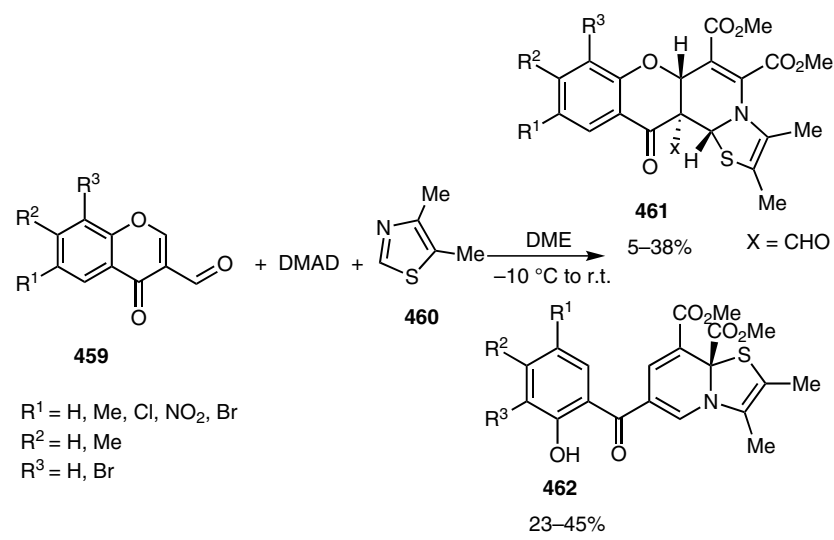

\section{Scheme 126}

Pyridines and quinolines generally deserve special attention owing to the variety of transformations that mediate. Following the observations made by Diels and Alder of the reaction of pyridine with DMAD (affording derivatives 465 and 466), ${ }^{157 a}$ Huisgen was successful in intercepting the 1,4-dipole 464 with phenyl isocyanate (467), leading to a pyrimidinedione derivative $\mathbf{4 7 0}$ with eventual elimination of pyridine during the course of the reaction (Scheme 127). ${ }^{157 b}$

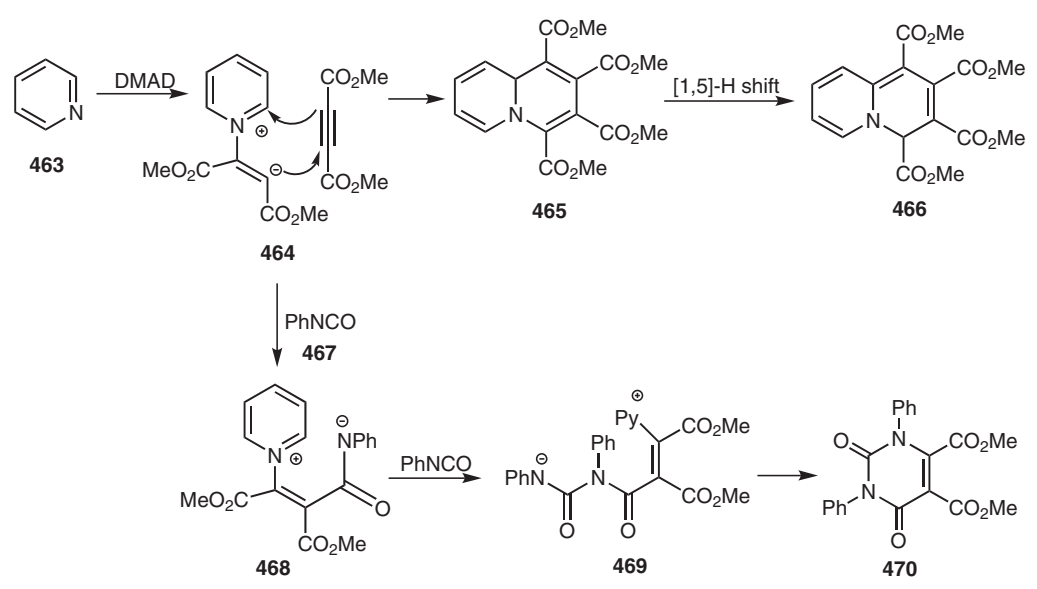

Scheme 127 


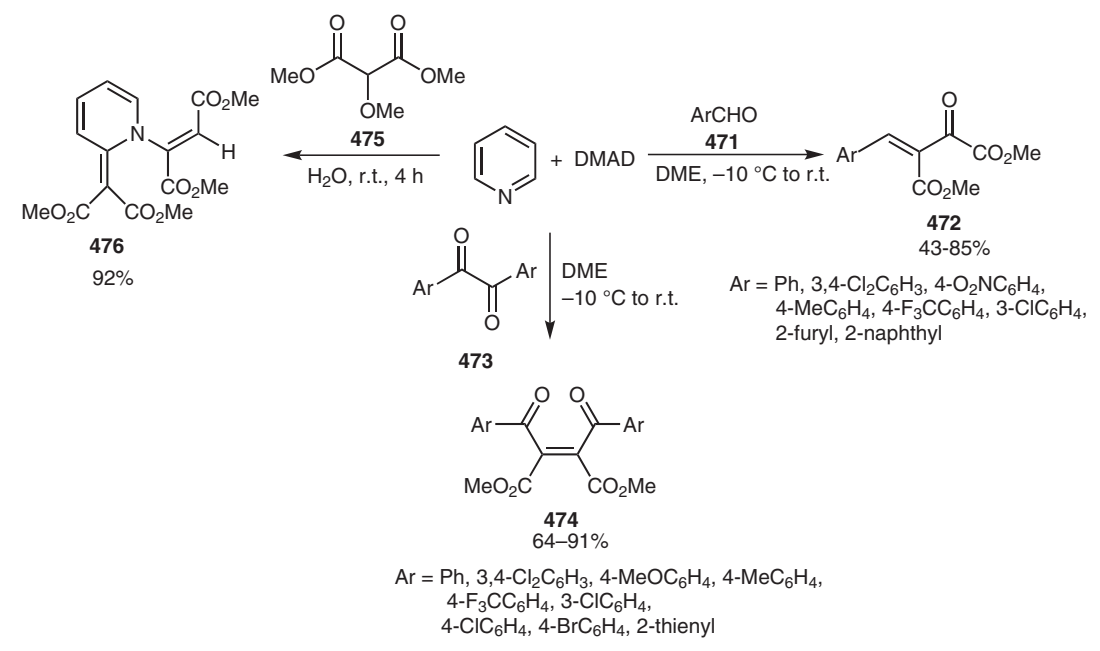

Scheme 128

The 1,4-zwitterionic intermediate generated from pyridine and DMAD was added to aldehydes 471 in a formal $[2+2]$ manner, resulting in the facile synthesis of 2-oxo-3benzylidenesuccinates 472 (Scheme 128). ${ }^{157 \mathrm{c}}$

Pyridine catalyzed the reaction of 1,2-diaryl diones $\mathbf{4 7 3}$ with DMAD to afford diaroyl maleates $\mathbf{4 7 4}$. This unprecedented rearrangement involved a unique benzoyl migration and proceeded with complete stereoselectivity (Scheme 128). ${ }^{158}$

In 2009, an efficient synthesis of the $2 H$-pyridinyl-2-butenedioate $\mathbf{4 7 6}$ was described via the reaction of dimethyl methoxymalonate (475) and DMAD in the presence of a nitrogen nucleophile (Scheme 128). ${ }^{159}$

Maghsoodlou et al. ${ }^{160}$ reported a three-component reaction between aromatic ketones 477 and DMAD in the presence of pyridines (Scheme 129).

A route towards stereoselective and regioselective halogenated pyrido[2,1-b][1,3]oxazines $\mathbf{4 8 1}$ in high yields was recently described by Asghari et al. ${ }^{161}$ The products were isolated in moderate to excellent yields through a three-component reaction involving pyridines 479, DMAD and different $\alpha$-halo ketones $\mathbf{4 8 0}$ (Scheme 130).

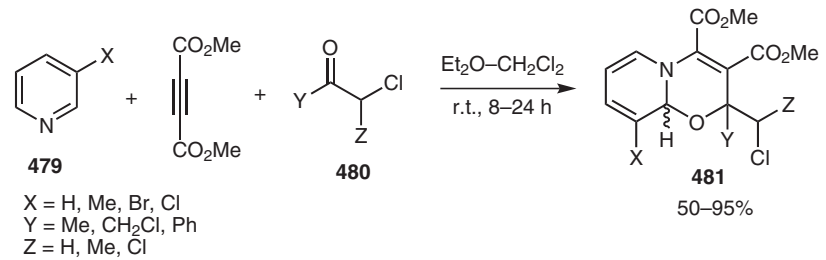

Scheme 130

The pyridine-mediated reaction of DMAD and cyclobutene-1,2-diones $\mathbf{4 8 2}$ afforded selective access to either hexasubstituted benzene derivatives $\mathbf{4 8 3}$ or cyclopentenedione derivatives 484 , depending on the concentration of pyridine (Scheme 131). ${ }^{162}$

Terzidis et al. ${ }^{163}$ reported an efficient synthesis of functionalized benzophenones $\mathbf{4 8 5}$, polysubstituted xanthones 486 or pyranochromenes 487 through the reaction of chromones 459 and DMAD catalyzed by a pyridine derivative (4-picoline or DMAP). The outcome of the reactions was found to depend on both the nature of the chromone substituents and the basicity of the organocatalyst (Scheme 132). Pyranochromenes 487 were also isolated by using DABCO or $\beta$-isoquinidine as organocatalysts. ${ }^{142}$
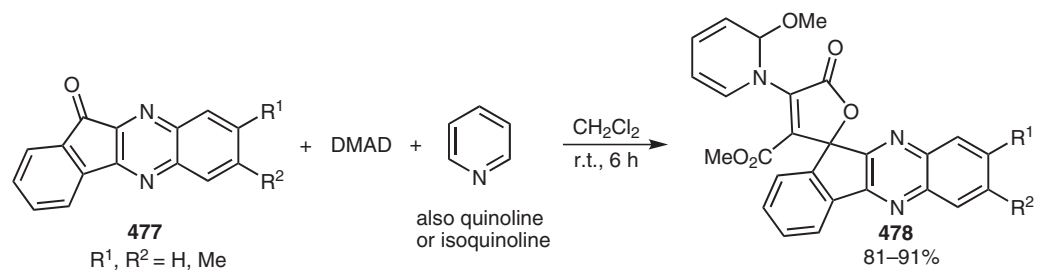

Scheme 129 


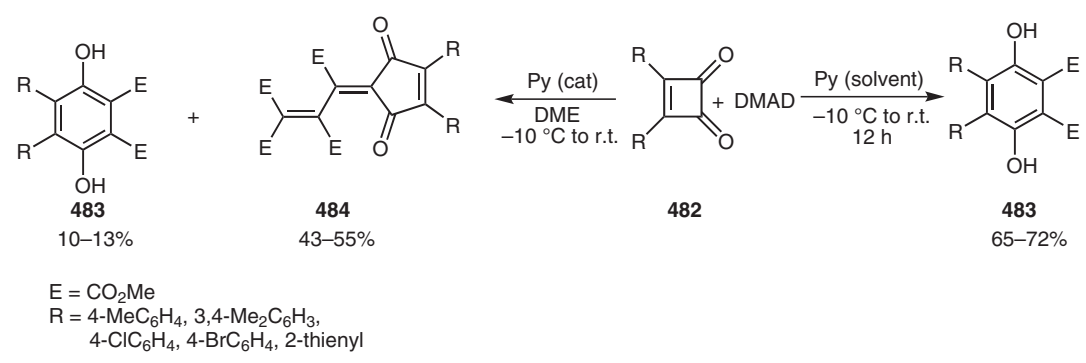

Scheme 131

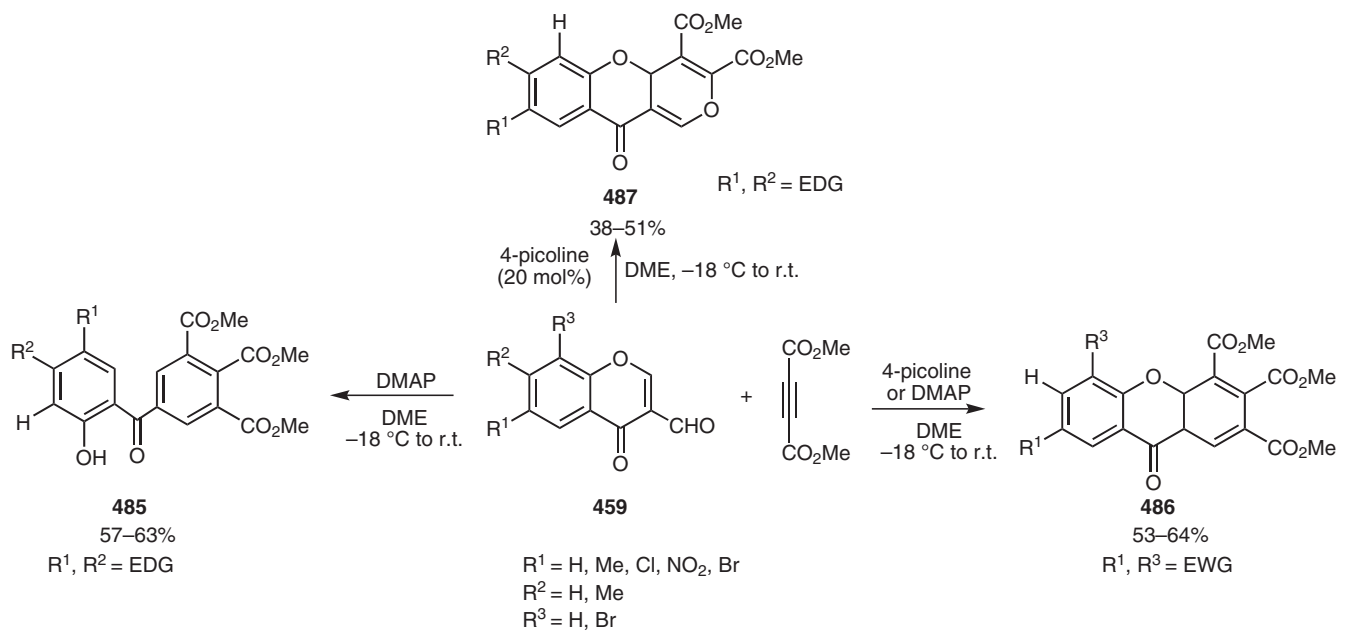

Scheme 132

Quinoline is widely known to form a 1,4-zwitterion with DMAD, which can be trapped by various dipolarophiles such as compounds 489, 491, 493, 495 and 497 to yield a variety of pyridoquinoline and oxazinoquinoline derivatives including 490, 492, 494, 496 and 498 and 499 (Scheme 133). ${ }^{164}$

The reaction of isoquinoline (500) with two equivalents of DMAD was originally developed by Diels and Alder in 1933. The reaction proceeded through the zwitterionic intermediate 501, which then underwent a domino Michael addition and Mannich reaction with a second equivalent of DMAD to afford benzoquinolizine 502 (Scheme 134). ${ }^{164 \mathrm{~b}}$

In 1967, Huisgen et al. ${ }^{165}$ reported three multicomponent variations of this reaction in which intermediate $\mathbf{5 0 1}$ was trapped with several different dipolarophiles, including dimethyl azodicarboxylate (503), diethyl mesoxalate (505) and phenyl isocyanate (507), to form the tricyclic scaffolds 504, 506 and 508, respectively (Scheme 134).

Later, some more examples were reported by Nair et al., ${ }^{166-168}$ who used benzoquinone 509 and arylidenemalononitriles 511 to obtain the spiro-isoquinoline 510 and tetrahydrobenzoquinolizine derivatives 512, respectively (Scheme 134).

More recently, Yavari et al. ${ }^{169}$ reported a new three-component reaction in which intermediate $\mathbf{5 0 1}$ was trapped with aroylnitromethanes $\mathbf{5 1 3}$ to give pyrroloisoquinolines 514 (Scheme 134). ${ }^{170}$

Multicomponent reactions involving azines [isoquinoline (500; Scheme 135) or phenanthridine (524; Scheme 136)] and DMAD were executed in the presence of heterocyclic $\mathrm{N}-\mathrm{H}$ compounds (indole, methylindole, 3,6-dibromocarbazole) or 1,3-dicarbonyl compounds $(N, N$-dimethylbarbituric acid, 1,3-diethyl-2-thiobarbituric acid, acetylacetone, 1,3-diphenylpropane-1,3-dione, cyclopentane-1,3-dione) $\mathbf{5 1 5}$ to generate enamino esters $\mathbf{5 1 6}$ and $\mathbf{5 2 6}$ in good yields. ${ }^{171,172}$

Isoquinoline reacted smoothly with DMAD in the presence of amides $\mathbf{5 1 7}$ to produce the derivatives $\mathbf{5 1 8}$ (Scheme 135). ${ }^{173}$

The 1,4-dipole derived from isoquinoline and DMAD was also shown to react readily with $N$-tosylimines $\mathbf{5 1 9}$, resulting in the diastereoselective synthesis of $2 \mathrm{H}$-pyrimido[2,1-a] isoquinoline derivatives 520 (Scheme 135). ${ }^{167}$ Moreover, the reaction of isoquinoline $\mathbf{5 0 0}$ and DMAD with benzoquinone 521, at room temperature, afforded the spiro-oxazino isoquinoline derivatives $\mathbf{5 2 2}$ and $\mathbf{5 2 3}$ as a mixture of regioisomers in a $2: 1$ ratio and $91 \%$ yield (Scheme 135). ${ }^{168}$

Li et al. ${ }^{174}$ described an efficient synthesis of [1,3]oxazino[3,2-f]phenanthridine derivatives $\mathbf{5 2 5}$ via a three- 


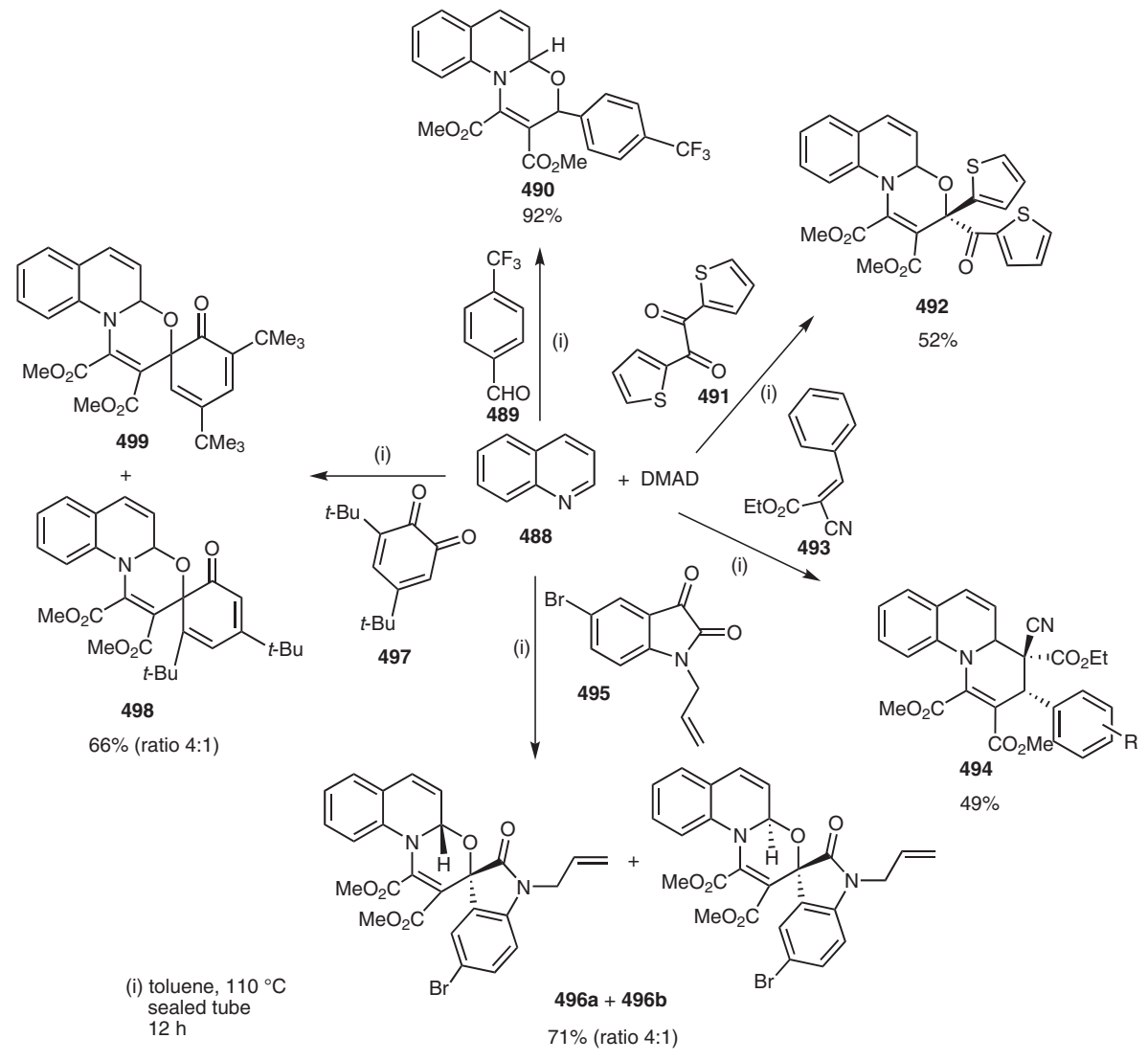

Scheme 133
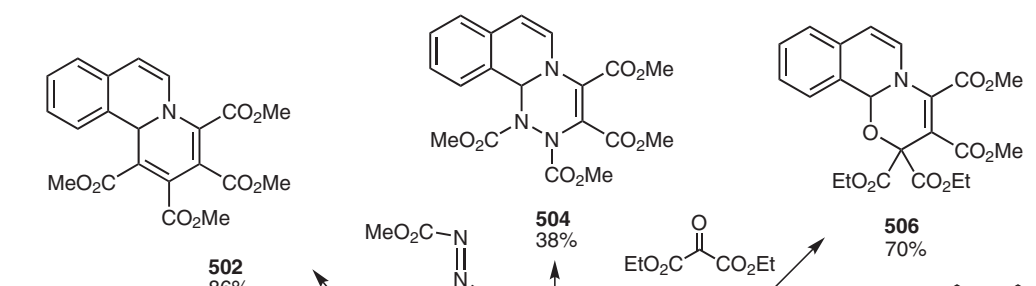

502
$86 \%$
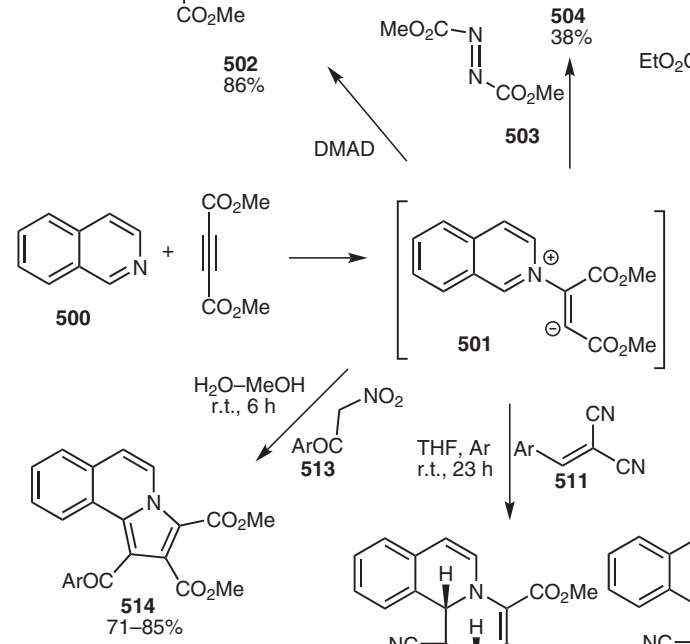

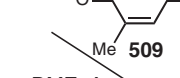$$
\text { DME, Ar }
$$

$\mathrm{DME}, \mathrm{Ar}$
r.t., $6 \mathrm{~h}$

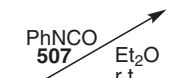

r.t.

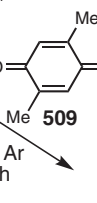

$$
\left.\mathrm{ArOC}_{513}^{\mathrm{NO}_{2}}{ }_{\text {r.t., } 23 \mathrm{Ar}}^{\mathrm{TH}}\right|_{511} ^{\mathrm{Ar}} \underset{\mathrm{CN}}{\mathrm{CN}}
$$

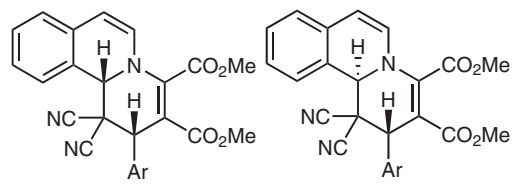

512a

$43-89 \%$

$512 b$

ratio from 100:0 to $1: 1$

Scheme 134 


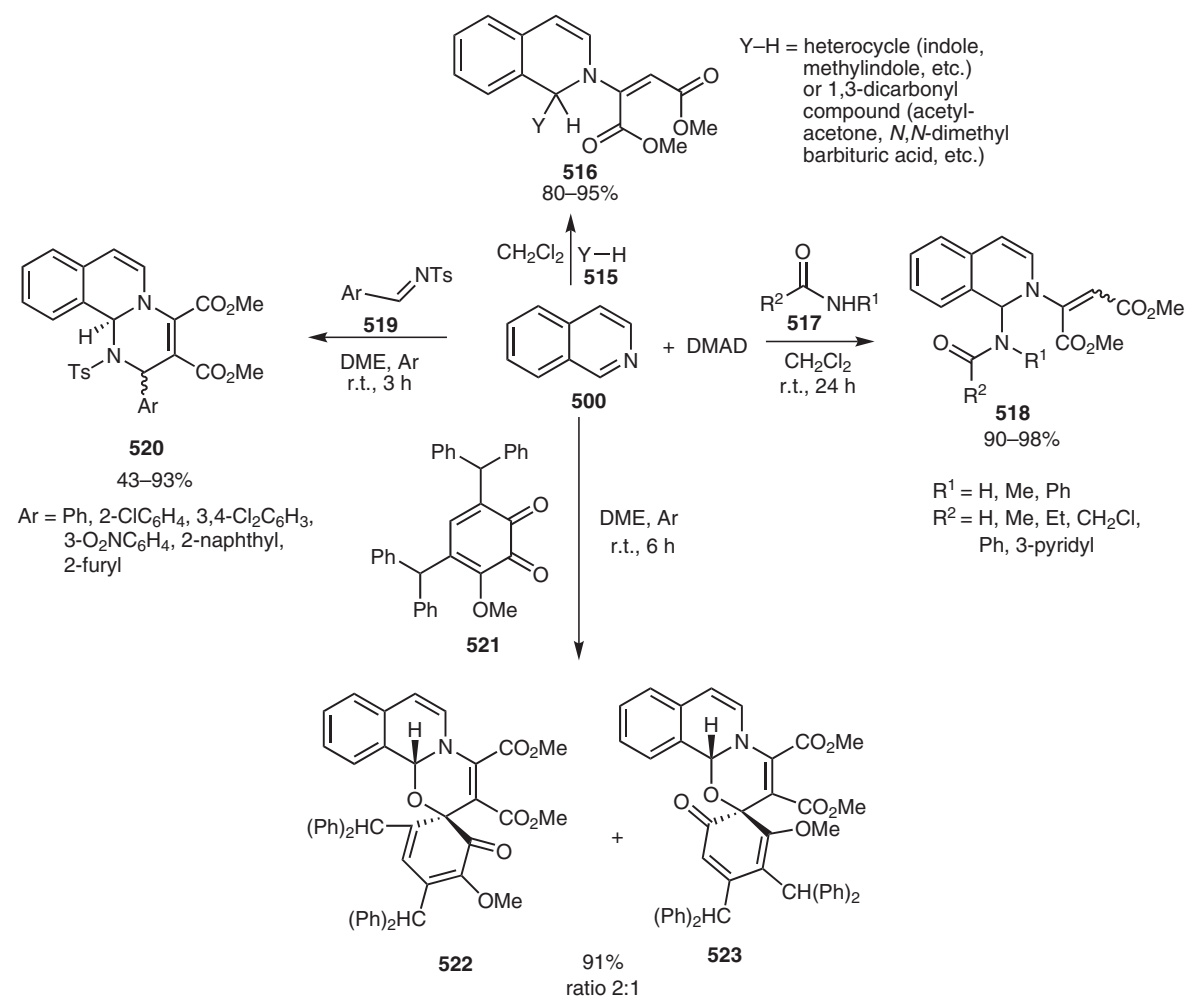

\section{Scheme 135}

component reaction of phenanthridine 524, DMAD and aromatic aldehydes (Scheme 136).

The reaction of 3-methylisoquinoline (527) with chromone-3-carboxaldehydes 459 and DMAD to produce chromenopyridoisoquinoline dicarboxylates $\mathbf{5 2 8}$ was also studied (Scheme 137). ${ }^{175}$

Safaei et al. ${ }^{176}$ reported a novel, facile and environmentally benign one-pot three-component synthesis of pyrazolines $\mathbf{5 3 0}$ from arylaldehydes, hydrazines $\mathbf{5 2 9}$ and DMAD

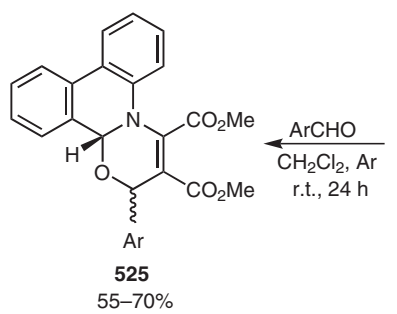
$\begin{aligned} \mathrm{Ar}= & 4-\mathrm{ClC}_{6} \mathrm{H}_{4}, 4-\mathrm{BrC}_{6} \mathrm{H}_{4}, 4-\mathrm{FC}_{6} \mathrm{H}_{4}, \\ & 2,4-\mathrm{Cl}_{2} \mathrm{C}_{6} \mathrm{H}_{3}, 4-\mathrm{MeC}_{6} \mathrm{H}_{4}, 2-\mathrm{FC}_{6} \mathrm{H}_{4} \\ & 3-\mathrm{FC}_{6} \mathrm{H}_{4}, 4-\mathrm{O}_{2} \mathrm{NC}_{6} \mathrm{H}_{4}, 2-\mathrm{BrC}_{6} \mathrm{H}_{4}\end{aligned}$

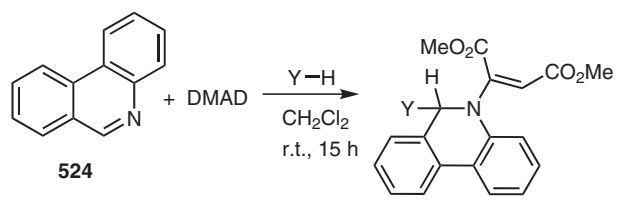

526

89-96\%

$\mathrm{Y}-\mathrm{H}=$ heterocycle (indole, methylindole, etc.) or 1,3-dicarbonyl acetone, $N, N$-dimethy barbituric acid, etc.)

\section{Scheme 136}

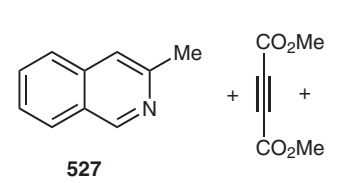

$\mathrm{R}^{1}=\mathrm{H}, \mathrm{Me}, \mathrm{Cl}, \mathrm{NO}_{2}, \mathrm{Br}$ $\mathrm{R}^{2}=\mathrm{H}, \mathrm{Me}$ $\mathrm{R}^{3}=\mathrm{H}, \mathrm{Br}$<smiles>[2H]c1cc2c(=O)c(C=O)coc2c([18OH])c1[18OH]</smiles>

459

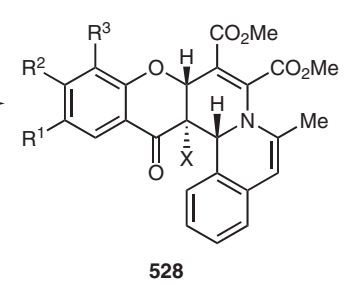

$\mathbf{5 2 8}$
$\mathrm{X}=\mathrm{CHO}$

Scheme 137 
with excellent yields and diastereoselectivities using a bifunctional Brønsted acidic ionic liquid as a safe, inexpensive and reusable catalyst under solvent-free conditions (Scheme 138).

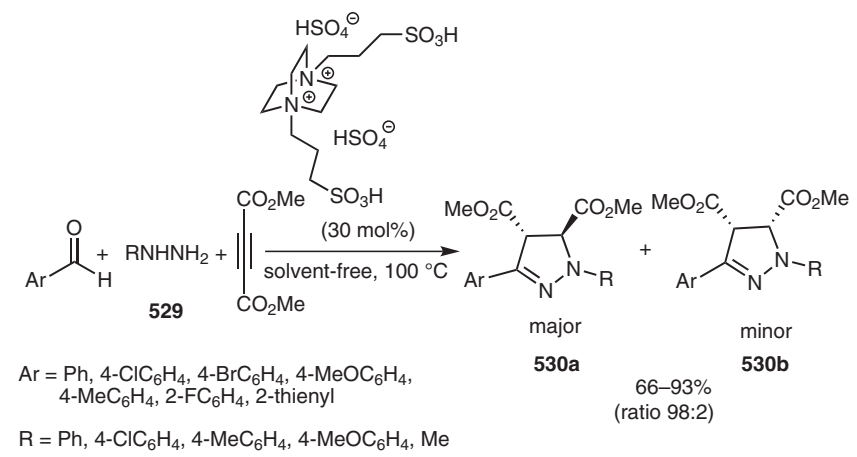

Scheme 138

\subsection{Isocyanides}

Hundreds of multicomponent reactions have been described over the years. Isocyanide-based multicomponent reactions (IMCRs) constitute a special subclass. They are particularly interesting because they are more versatile and diverse than the other multicomponent reactions. The great potential of isocyanides for the development of multicomponent reactions lies in the diversity of bond-forming processes available, their functional group tolerance and the high levels of chemo-, regio- and stereoselectivity often observed. The outstanding position of IMCRs can be traced back to the exceptional reactivity of the functional group of the isocyanides. No other functional group reacts with both nucleophiles and electrophiles on the same atom, leading to the so-called R-adduct. Winterfeldt et al. were the first to describe the reactions of DMAD with isocyanides in their pioneering work published in 1969. ${ }^{177 a}$ A large number of IMCRs were described by Dömling in his reviews. ${ }^{177 b, c}$

\subsubsection{Synthesis of Five-Membered Heterocycles with One Heteroatom}

\subsubsection{Nitrogen-Containing Heterocycles}

The zwitterion generated by the addition of alkyl(aryl) isocyanides 531 to DMAD was trapped by benzoyl chloride (532) to yield functionalized 2,5-dihydro-1 $H$-pyrroles 533 (Scheme 139). However, in the presence of electron-withdrawing groups at the para-position of the benzoyl chloride, tetrasubstituted furans $\mathbf{5 3 4}$ were isolated instead. ${ }^{178}$

The 1:2 zwitterion, generated by the addition of triphenylphosphine to DMAD, was protonated by trifluoroace-

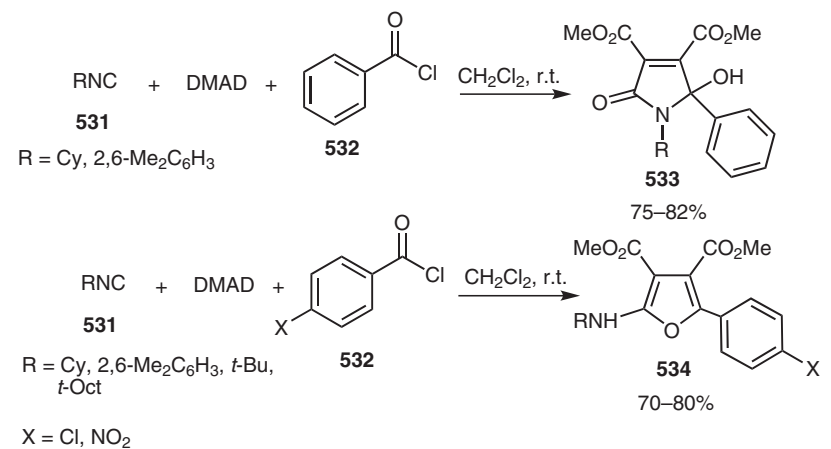

Scheme 139

tic acid and subsequently attacked by isocyanide and water in a pseudo-seven-component diastereoselective reaction giving compounds $\mathbf{5 3 5}$ with three stereogenic centers and a phosphorane group in good yields (Scheme 140). ${ }^{179}$

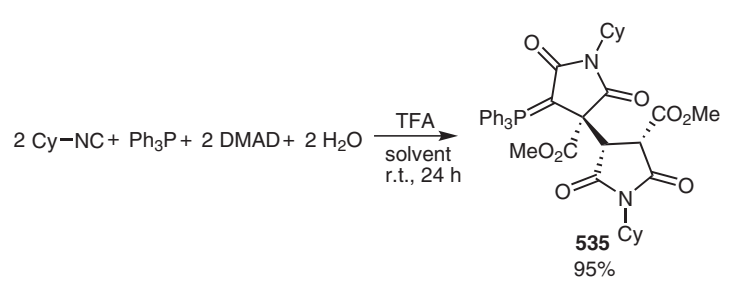

\section{Scheme 140}

The three-component reaction of the zwitterion generated from DMAD and isocyanides with various quinoneimides such as $\mathbf{5 3 6}$ and $\mathbf{5 3 8}$ afforded the corresponding $\gamma$-spiroiminolactams 537 and 539 in good yields (Scheme 141). ${ }^{180}$

\subsubsection{Oxygen-Containing Heterocycles}

In 2004, the three-component reaction of cyclohexyl isocyanide with DMAD and various aromatic or aliphatic aldehydes 540 was reported to have gone to completion in less than two hours when carried out in ionic liquids, affording the expected heterocycles 542 in high yields. ${ }^{181}$ Water was reported as a novel reaction medium for the synthesis of highly functionalized 2-aminofuran derivatives 542, via the coupling of aldehydes 540, with DMAD and cyclohexyl isocyanide (Scheme 142). ${ }^{182} 3$-Aromatic aldehydes, ${ }^{183 \mathrm{a}}$ formylindoles ${ }^{183 \mathrm{~b}}$ and quinoline-3-carbaldehydes ${ }^{183 \mathrm{c}}$ were also found to undergo smooth condensation with the zwitterions derived from isocyanides and DMAD either in benzene or in acetonitrile to give the corresponding furanyl derivatives in good yields. 


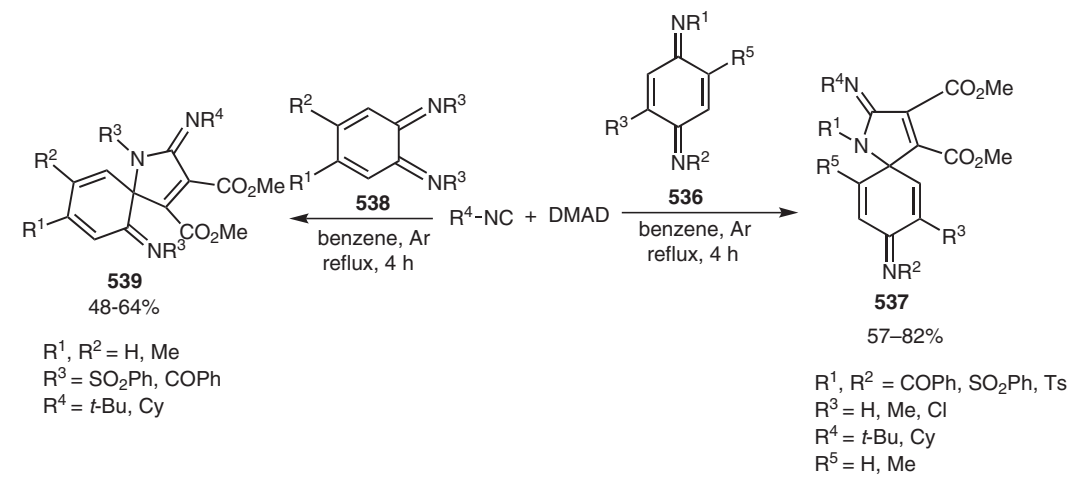

Scheme 141

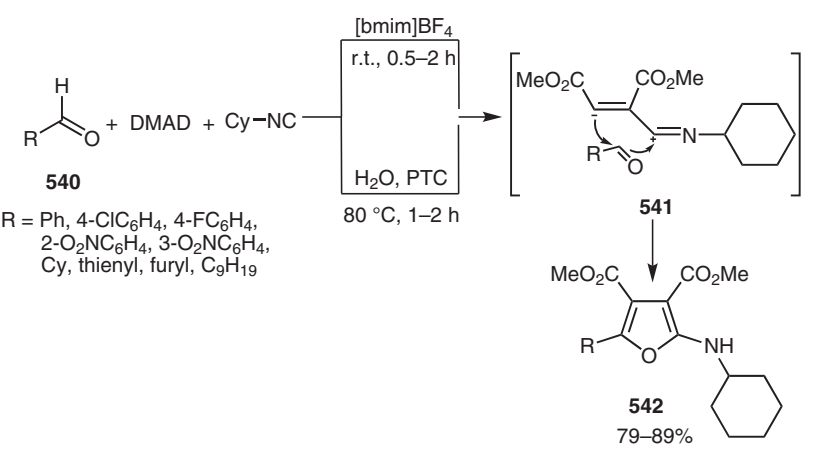

Scheme 142

In addition, the reaction of 1:1 zwitterionic intermediates generated in situ from either tert-butyl isocyanide or cyclohexyl isocyanide and DMAD with 3-formylchromones 459 was reported to lead to chromenylfurandicarboxylates 543 or to cyclopenta $[b]$ chromenedicarboxylates 544 , depending on the nature of the chromone 6-position substituent (Scheme 143). ${ }^{184}$

The reaction of alkyl isocyanides with DMAD, in the presence of pyridine-containing carbonyl compounds $\mathbf{5 4 5}$ or $\mathbf{5 4 7}$, led to the stable products $\mathbf{5 4 6}$ or $\mathbf{5 4 8}$ in excellent yields (Scheme 144). ${ }^{185}$

The reaction of DMAD and isocyanides with vicinal tricarbonyl systems 549 and 550 produced highly substituted furan derivatives $\mathbf{5 5 1}$ and $\mathbf{5 5 2}$ respectively, whereas when the diphenyl triketone $\mathbf{5 5 3}$ was used, the pyran derivative $\mathbf{5 5 4}$ was the only reaction product (Scheme 145). ${ }^{186}$

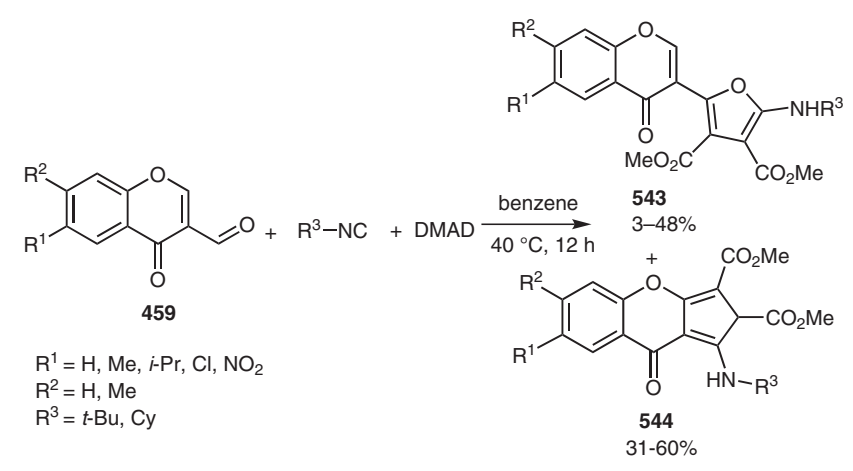

Scheme 143

The zwitterion formed from an alkyl isocyanide and DMAD reacted with acetic anhydride (555) or phthalic anhydride (556) to form methylfurans or benzo-fused spirolactones $\mathbf{5 5 7}$ or $\mathbf{5 5 8}$ in relatively good yields, at room temperature and without using a catalyst (Scheme 145). ${ }^{187}$

Reaction of tert-butyl isocyanide with DMAD in the presence of 2-acetylbutyrolactone (559) led to the formation of the furanylidenebutenedioate $\mathbf{5 6 0}$ (Scheme 145). ${ }^{188}$

The reaction between alkyl isocyanides and phenanthrene-9,10-dione (561) or dione $\mathbf{5 6 3}$ in the presence of DMAD was found to afford $\gamma$-dispiroiminolactones $\mathbf{5 6 2}$ and 564, respectively, in high yields (Scheme 146). ${ }^{189}$

1,2-Benzoquinones are inert towards isocyanides and electron-deficient alkynes at ambient temperature; however, they readily react with the zwitterions generated from these two. For example, the reaction of cyclohexyl isocyanide and DMAD generated a zwitterion which, on

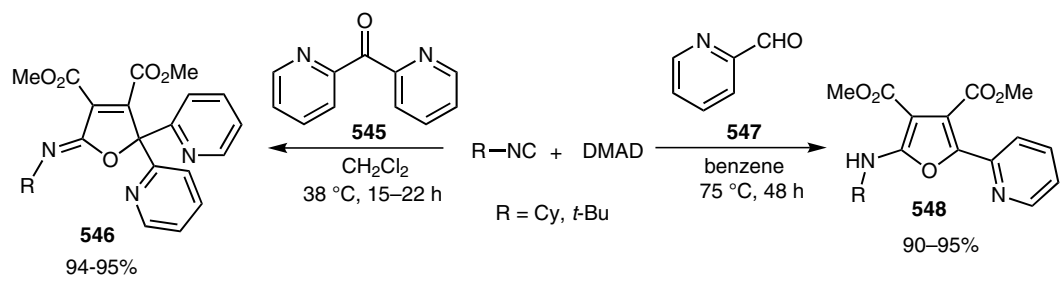

Scheme 144 

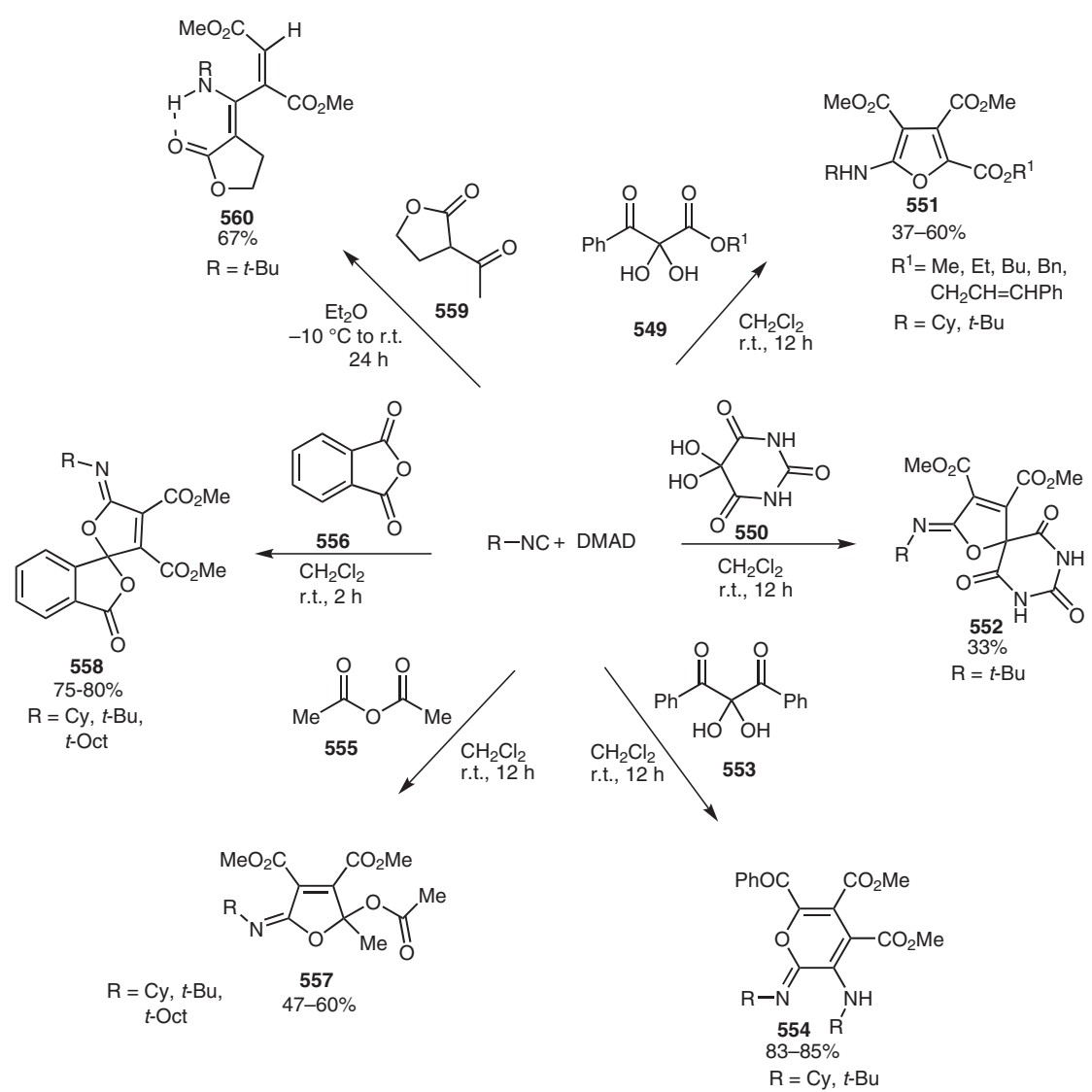

\section{Scheme 145}

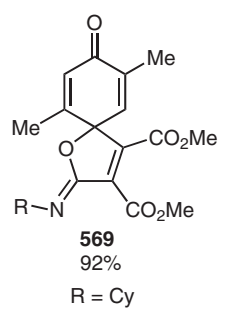<smiles>CC1=CC(=O)C(C)=CC1=O</smiles>

568

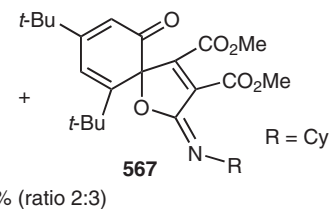

$58 \%$ (ratio $2: 3$ )<smiles>CC(C)(C)C1=CC(=O)C(=O)C(C(C)(C)C)=C1</smiles>

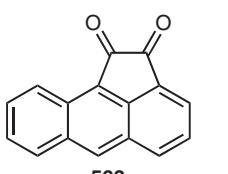

$\mathrm{CH}_{2} \mathrm{Cl}_{2}$ $38^{\circ} \mathrm{C}, 48 \mathrm{~h}$
$\mathrm{C}$

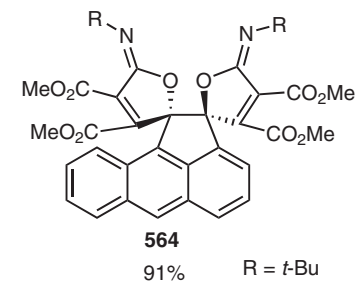

$91 \% \quad \mathrm{R}=t-\mathrm{Bu}$

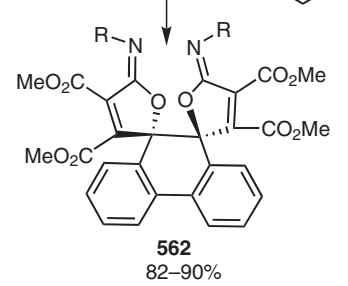

$\mathrm{R}=\mathrm{Cy}, \mathrm{Bn}, t-\mathrm{Bu}$

\section{Scheme 146}


interception with 3,5-di-tert-butyl-1,2-benzoquinone (565), yielded a regioisomeric mixture of spiroiminolactones $\mathbf{5 6 6}$ and $\mathbf{5 6 7}$ reacting exclusively with the carbonyl functionalities of the quinone (Scheme 146). ${ }^{190}$

Nair et al. ${ }^{191}$ found that DMAD could be induced to add itself to the most electron-deficient carbonyl of various benzoquinones in the presence of some nucleophilic initiator, such as triphenylphosphine or an isonitrile. In particular, benzoquinone 568 underwent cyclization in the presence of cyclohexyl isocyanide and DMAD to afford the iminolactone 569 in $92 \%$ yield (Scheme 146). ${ }^{192}$

Isocyanides reacted smoothly with DMAD in the presence of hexachloroacetone (570) to produce the furan derivatives 571 in high yields (Scheme 147). ${ }^{193}$

Benzoyl chlorides 572 with electron-withdrawing substituents at the para-position led to tetrasubstituted furans 573 (Scheme 147). ${ }^{178}$

A three-component condensation reaction between an isocyanide, DMAD and 2-bromo-1-(4-bromophenyl)ethanone (574) efficiently provided fully substituted iminolactones 575 in high yields in a one-pot condensation reaction without any activation or modification (Scheme 147). ${ }^{194}$
Moreover, a new and efficient method for preparing electron-poor imides $\mathbf{5 7 7}$ and fully substituted furans $\mathbf{5 7 8}$ from triphenylphosphine, 1,1,3,3-tetramethylbutyl isocyanide, DMAD and benzoic acid (576) under neutral conditions has been reported (Scheme 147). ${ }^{195}$

The reaction between alkyl(aryl) isocyanides, DMAD and alkyl cyanoformates $\mathbf{5 7 9}$ under solvent-free conditions led to furan derivatives $\mathbf{5 8 0}$ in high yields (Scheme 147). ${ }^{196}$

The highly reactive 1:1 adducts produced from the reaction between DMAD and alkyl isocyanides were trapped by benzoyl cyanide derivatives $\mathbf{5 8 1}$ to afford furan derivatives $\mathbf{5 8 2}$ in good yields (Scheme 147). ${ }^{197}$

\subsubsection{Synthesis of Six-Membered Heterocycles with One Heteroatom}

\subsubsection{Nitrogen-Containing Heterocycles}

A three-component condensation reaction between an isocyanide, DMAD and triphenylphosphonium bromide $\mathbf{5 8 3}$ efficiently provided fully substituted $N$-alkyl-2-triphenylphosphoranylidene glutarimides $\mathbf{5 8 4}$ in a one-pot reaction without any activation (Scheme 148). ${ }^{198}$

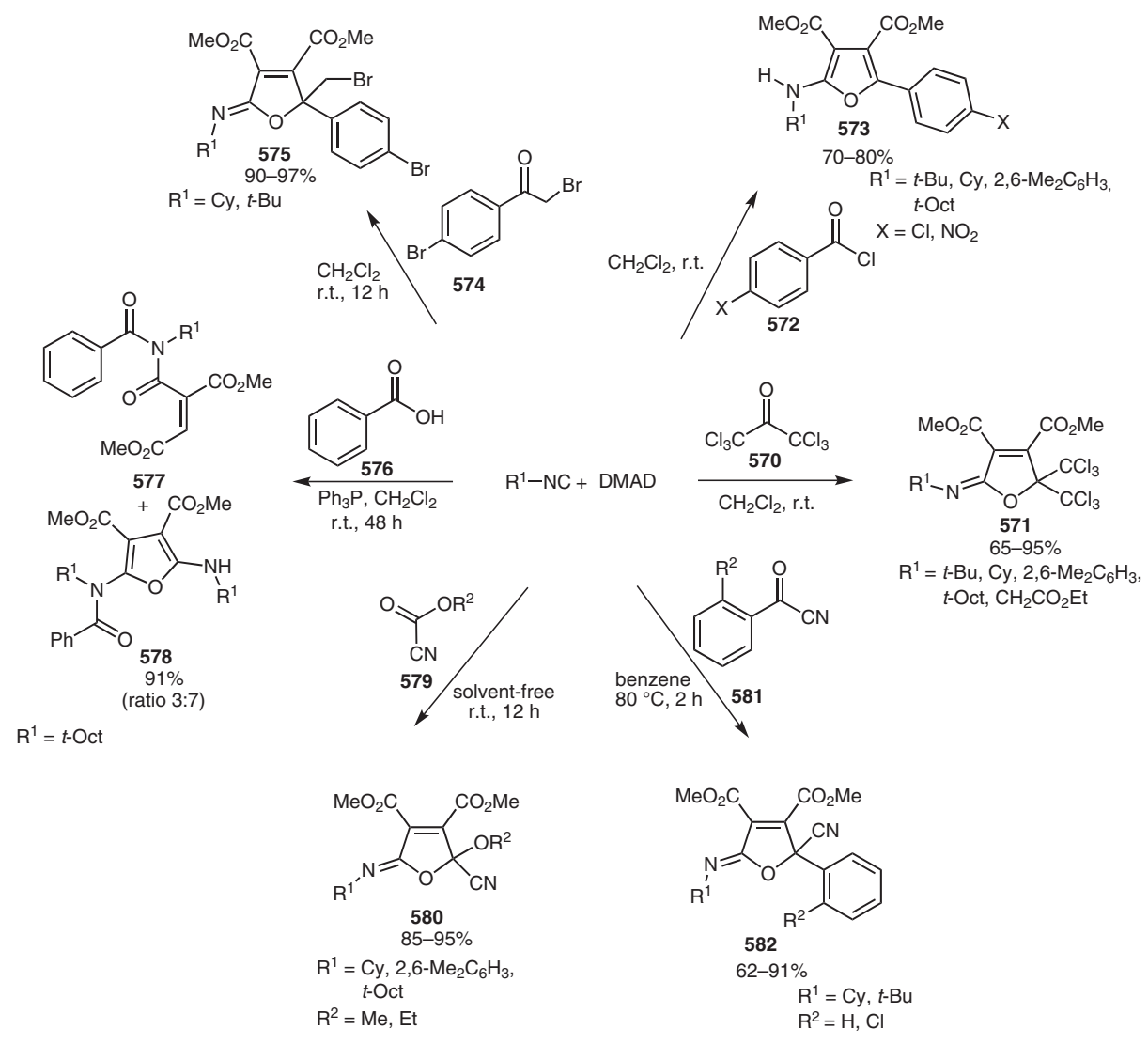

Scheme 147 


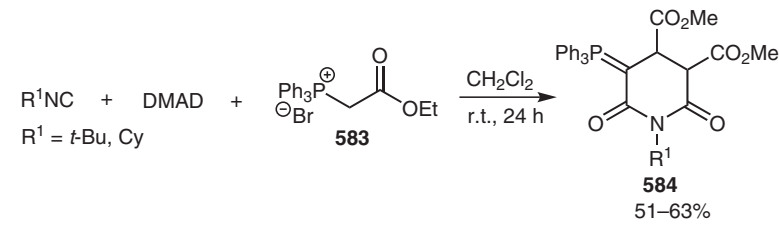

Scheme 148

Li et al. ${ }^{199}$ reported a facile, efficient and regioselective synthetic approach for the construction of highly substituted pyridine-2(1H)-ones $\mathbf{5 8 6}$ and allenyl derivatives 587. Their synthesis involved a one-pot three-component reaction between $N$-arylidene-2-cyanoacetohydrazides 585, DMAD and isocyanides (Scheme 149).

\subsubsection{Oxygen-Containing Heterocycles}

Functionalized dihydroindenopyrans 589 were synthesized from the reaction of alkyl(aryl) isocyanides, DMAD and indane-1,3-dione (588; Scheme 150). ${ }^{200}$

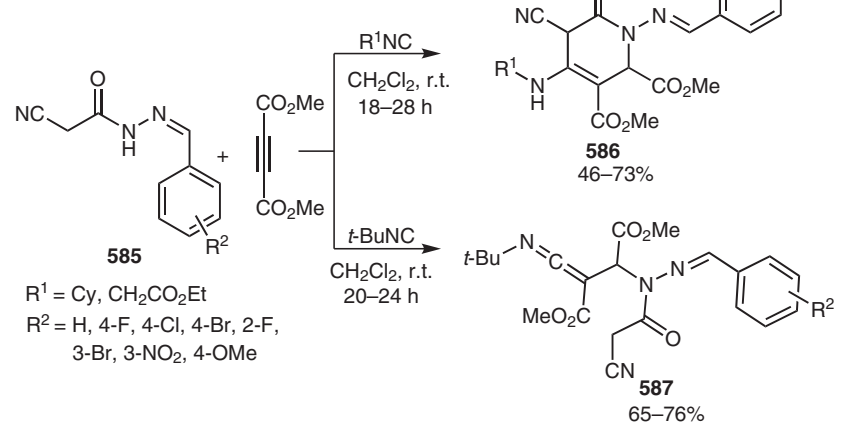

Scheme 149

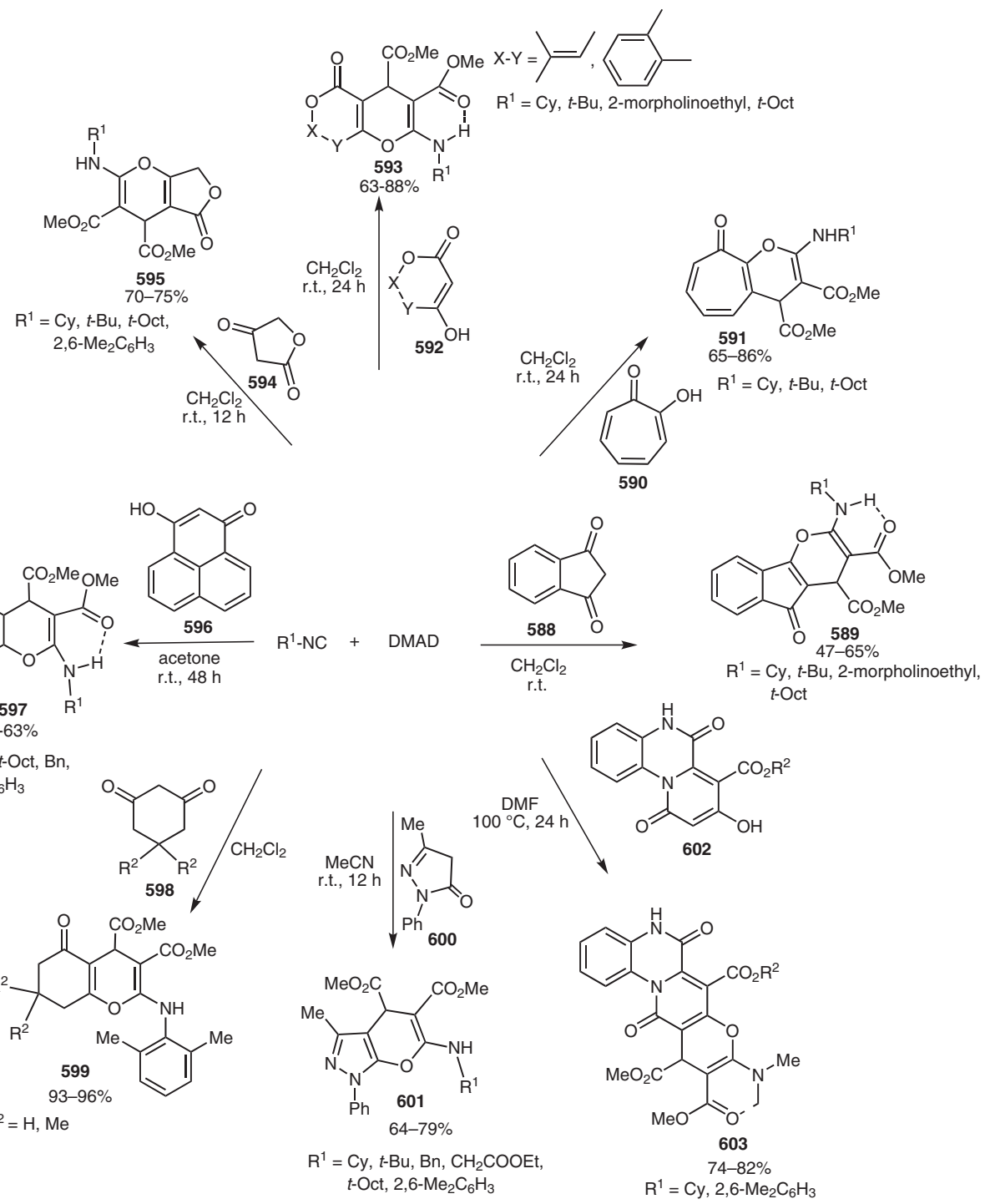

\section{Scheme 150}


Fused heterocycles were prepared in a one-pot three-component reaction of alkyl isocyanide, DMAD and $\alpha$-tropolone $\mathbf{( 5 9 0 )}$. The reaction proceeded smoothly at room temperature and under neutral conditions to afford tropolone derivatives 591 in good to high yields (Scheme 150). ${ }^{201}$

Chemoselective reaction of isocyanides with DMAD in the presence of relatively strong cyclic $\mathrm{C}-\mathrm{H}$ acids 592, such as 4-hydroxy-6-methyl-2H-pyran-2-one or 4-hydroxycoumarin, led to a facile synthesis of highly functionalized chromeno or pyrano derivatives 593, respectively, in good yields (Scheme 150). ${ }^{202}$

A three-component reaction of an isocyanide, DMAD and tetronic acid (594) in dichloromethane at room temperature afforded 4H-furo[3,4-b]pyran derivatives $\mathbf{5 9 5}$ (Scheme 150). ${ }^{203}$

The reaction between alkyl or aryl isocyanides and DMAD with 3-hydroxy-1H-phenalene-1-one 596 produced a vinylisonitrilium cation, which subsequently underwent an addition reaction with the conjugate base of the 3-hydroxy-1H-phenalene-1-one to produce biologically interesting compounds $\mathbf{5 9 7}$ in moderate to fairly good yields (Scheme 150). ${ }^{204}$

The reaction between 2,6-dimethylphenyl isocyanide, 1,3-cyclohexanediones 598 and DMAD provided a simple one-pot entry into the synthesis of polyfunctional $4 \mathrm{H}$ chromene derivatives 599 (Scheme 150). ${ }^{205}$

A three-component reaction of isocyanides, DMAD and 3-methyl-1-phenyl-1H-pyrazol-5(4H)-one $\mathbf{6 0 0}$ led to the synthesis of fully substituted pyrano[2,3-c]pyrazole derivatives 601 (Scheme 150). ${ }^{206}$

Pyrano-pyrido quinoxaline derivatives 603 were synthesized in good yields by a three-component reaction of isocyanides, DMAD and pyrido[1,2-a]quinoxalinetriones $\mathbf{6 0 2}$ in $N, N$-dimethylformamide at $100^{\circ} \mathrm{C}$ (Scheme 150)..$^{207}$

\subsubsection{Synthesis of Six-Membered Heterocycles with Two Heteroatoms}

\subsubsection{Nitrogen-Containing Heterocycles}

Reaction of alkyl isocyanides, DMAD and dimethylurea (604) in glucose provided novel 2,6-dioxohexahydropyrimidines 605 (Scheme 151). ${ }^{208}$

A one-pot, three-component synthesis of pyrimidine derivatives 607, from the reaction of isocyanides, DMAD and $\mathrm{N}$-(2-heteroaryl)amides $\mathbf{6 0 6}$, was also reported (Scheme 151). ${ }^{209}$

A three-component reaction of isocyanides, DMAD and $N$-(2-pyridyl)amides 608 led to the synthesis of the corresponding $4 H$-pyrido[1,2-a]pyrimidines $\mathbf{6 0 9}$ (Scheme 151). ${ }^{210}$

The one-pot, three-component condensation reaction of alkyl isocyanides with DMAD in the presence of phthalhydrazide 610 was successfully applied to the synthesis of compounds 611 (Scheme 151). ${ }^{211}$

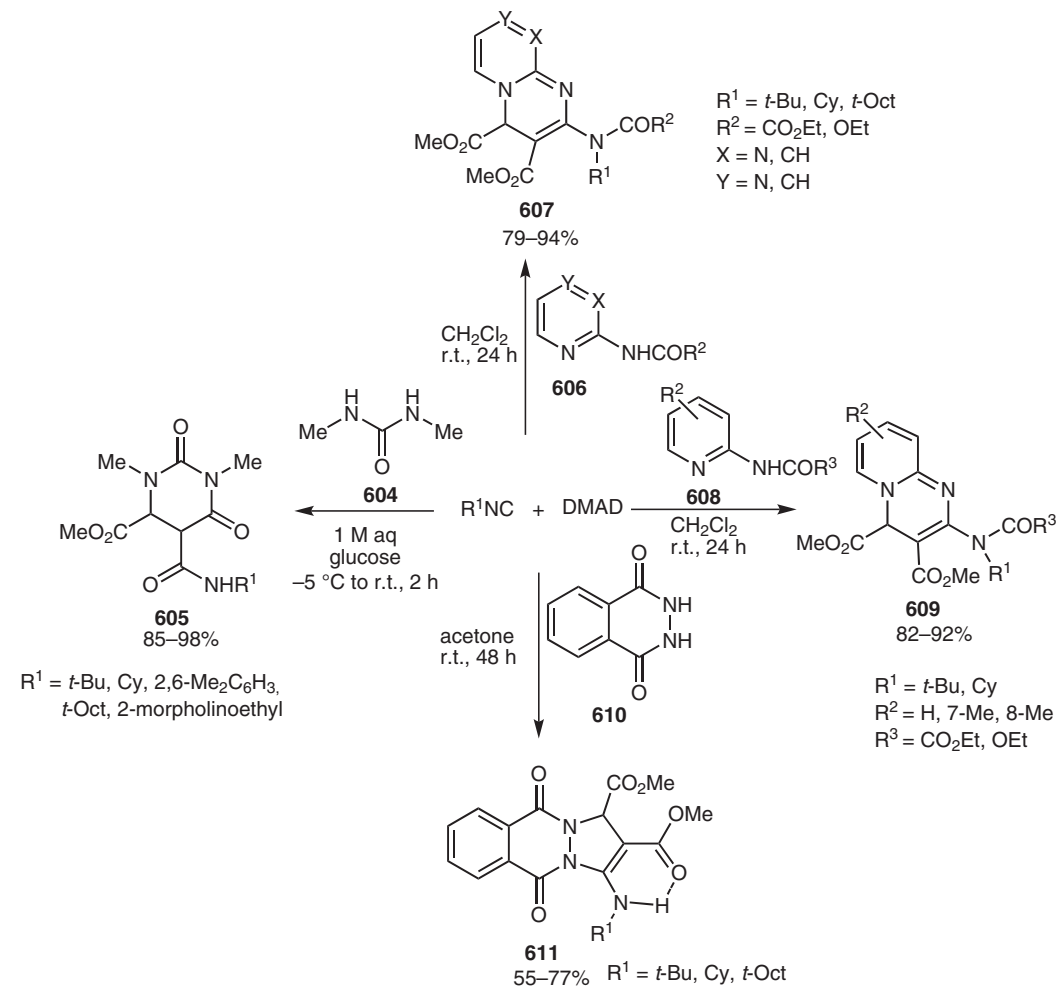

Scheme 151 


\subsection{Carbenes}

Over the past half-century and specifically ever since Breslow's original demonstration of the role of thiazole carbenes as nucleophilic catalysts in enzymatic reactions, ${ }^{212}$ the intensive studies of N-heterocyclic carbenes (NHCs) as reaction intermediates by Wanzlick ${ }^{213}$ and the first isolation of stable diaminocarbene by Arduengo and coworkers in 1991,214 these species have attracted considerable attention. Their role as excellent ligands for transition metals ${ }^{215}$ and their ability to catalyze various carbon-carbon coupling reactions, namely benzoin condensation, transesterification ${ }^{216}$ and Stetter reaction, ${ }^{217}$ have contributed significantly to the tremendous interest in N-heterocyclic carbenes.

A straightforward preparation of 3-aminofuran derivatives 614 via multicomponent reactions of thiazole carbenes 612, aldehydes 613 and DMAD was reported. In this process, the thiazole carbenes, generated in situ from thiazolium salts, reacted with aldehydes and DMAD to afford the substituted furans $\mathbf{6 1 4}$ in moderate to good yields. Eight substituted thiazolium salts were employed as carbene precursors in the reaction. In addition to aryl aldehydes, $\alpha, \beta$-unsaturated aldehydes $\mathbf{6 1 5}$ were also investigated and found to be applicable to this reaction (Scheme 152). ${ }^{218}$

A three-component synthesis of the unique polysubstituted furan-fused 1,4-thiazepines $\mathbf{6 1 8}$ from thiazolium salts 612, 1,1-disubstituted ketenes 617 and DMAD was also reported (Scheme 152). ${ }^{219}$

Nair et al. ${ }^{220}$ described the reaction of carbenes $\mathbf{6 2 0}$ with DMAD and aromatic aldehydes 619, which proceeded smoothly to deliver four-component acyclic adducts $\mathbf{6 2 1}$ in good yields (Scheme 153).

$$
\begin{aligned}
& \text { } \\
& \mathrm{Ar}=3,4-\mathrm{Cl}_{2} \mathrm{C}_{6} \mathrm{H}_{3}, 3-\mathrm{ClC}_{6} \mathrm{H}_{4} \\
& 4-\mathrm{BrC}_{6} \mathrm{H}_{4}, 4-\mathrm{F}_{3} \mathrm{CC}_{6} \mathrm{H}_{4} \text {, } \\
& \text { 4- }{ }_{2} \mathrm{NC}_{6} \mathrm{H}_{4}, 4-\mathrm{MeC}_{6} \mathrm{H}_{4} \\
& \mathrm{R}=t-\mathrm{Bu}
\end{aligned}
$$

Scheme 153

1-Thiocarbamoylimidazo[1,5-a]pyridinium inner salts 624, which were obtained readily from the addition of the $\mathrm{C}, \mathrm{N}$-substituted heterocyclic carbenes, imidazo[1,5a]pyridin-1-ylidenes $\mathbf{6 2 2}$, to isothiocyanates $\mathbf{6 2 3}$, are powerful ambident nucleophilic zwitterions. When treated with DMAD, they behaved exclusively as sulfur nucleophiles to afford fully substituted thiophenes $\mathbf{6 2 5}$ in excellent yields, providing an efficient orthogonal synthesis of polyfunctionalized thiophenes not easily obtained by other chemical means (Scheme 154). ${ }^{221}$

Pan et al. ${ }^{222}$ reported a multicomponent reaction using both N-heterocyclic carbenes and substituted phthalaldehydes. The imidazo[1,5- $a$ ]pyridine carbenes $\mathbf{6 2 6}$ reacted with phthalaldehydes 627 and DMAD to produce diastereomeric benzo $[d]$ furo[3,2-b]azepine derivatives $\mathbf{6 2 8}$ and 629. The carbene underwent a nucleophilic addition to the phthalaldehyde to form a dipolar intermediate which then underwent [3+2] cycloaddition with DMAD (Scheme 155).

\section{Scheme 152}




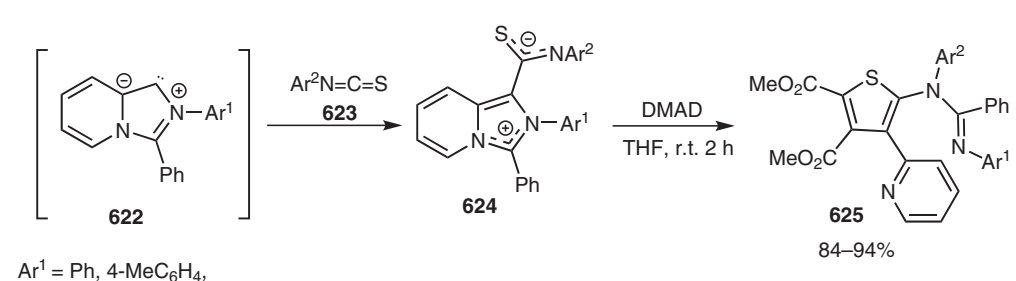

$\mathrm{Ar}^{1}=\mathrm{Ph}, 4-\mathrm{MeC}_{6} \mathrm{H}_{4}$,

4- $\mathrm{MeOC}_{6} \mathrm{H}_{4}, 4-\mathrm{ClC}_{6} \mathrm{H}_{4}$

$\mathrm{Ar}^{2}=\mathrm{Ph}, 4-\mathrm{BrC}_{6} \mathrm{H}_{4}, 4-\mathrm{MeOC}_{6} \mathrm{H}_{4}$

Scheme 154
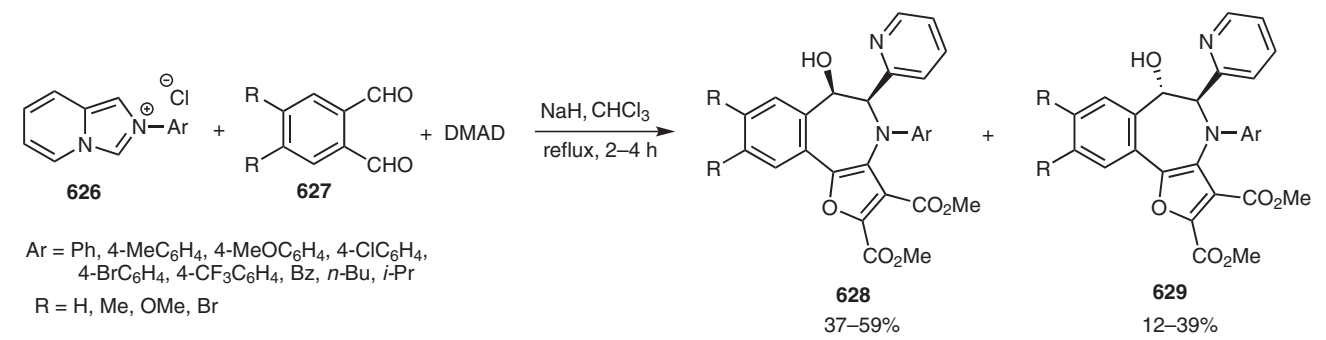

Scheme 155

\subsection{Miscellaneous Reactions}

Chaniyara et al., ${ }^{223}$ in continuation of their research into new bifunctional DNA-crosslinking agents for antitumor application, used benzothiazole derivative $\mathbf{6 3 0}$ and DMAD in order to gain access to diesters $\mathbf{6 3 1}$ and ultimately, after two steps, to the bis(alkylcarbamate) derivatives $\mathbf{6 3 2}$ for antitumor studies (Scheme 156).

In 2011, an efficient synthesis of iminothiopyran and isothiochromene derivatives 635 and 637, via one-pot reactions between DMAD, aryl isothiocyanates $\mathbf{6 3 3}$ and enaminones 634 and 636 in dichloromethane at room temperature, was described (Scheme 157). ${ }^{24}$

The zwitterion formed by the reaction of dimethoxycarbene and DMAD added efficiently to one of the carbonyl groups of 1,2-dicarbonyls and anhydrides to generate dihydrofurans and spirodihydrofurans 639-642 in good yields. In many cases, the carbene is inserted into the carbon-carbon bond of the dione to yield masked vicinal tricarbonyl systems (Scheme 158).225

Ding et al. ${ }^{226}$ reported a 1-methylimidazole-catalyzed reaction of DMAD with in situ generated ketenes by the action of Hunig's base on acyl halides (Scheme 159).

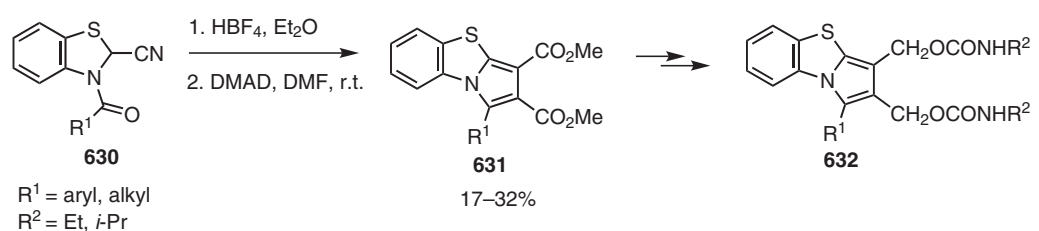

Scheme 156
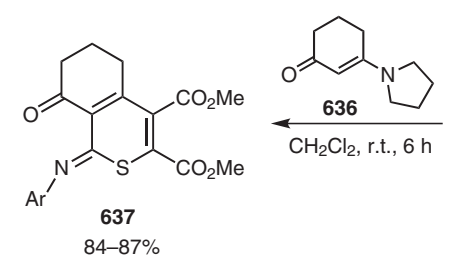

$\mathrm{Ar}-\mathrm{N}=\mathrm{C}=\mathrm{S}+\mathrm{DMAD}$ 633

$\mathrm{Ar}=\mathrm{Ph}, 4-\mathrm{O}_{2} \mathrm{NC}_{6} \mathrm{H}_{4}$, 4- $\mathrm{BrC}_{6} \mathrm{H}_{4}, 2-\mathrm{FC}_{6} \mathrm{H}_{4}$ $3-\mathrm{BrC}_{6} \mathrm{H}_{4}, 3-\mathrm{FC}_{6} \mathrm{H}_{4}$,
$4-\mathrm{MeC}_{6} \mathrm{H}_{4}, 4-\mathrm{MeOC}_{6} \mathrm{H}_{4}$<smiles>CC(=O)C=C(C)N1CCCC1</smiles><smiles>CC(=O)c1c([N+](=O)[O-])s/c(=N/O)c(C(C)=O)c1C(C)=O</smiles>

835

Scheme 157 


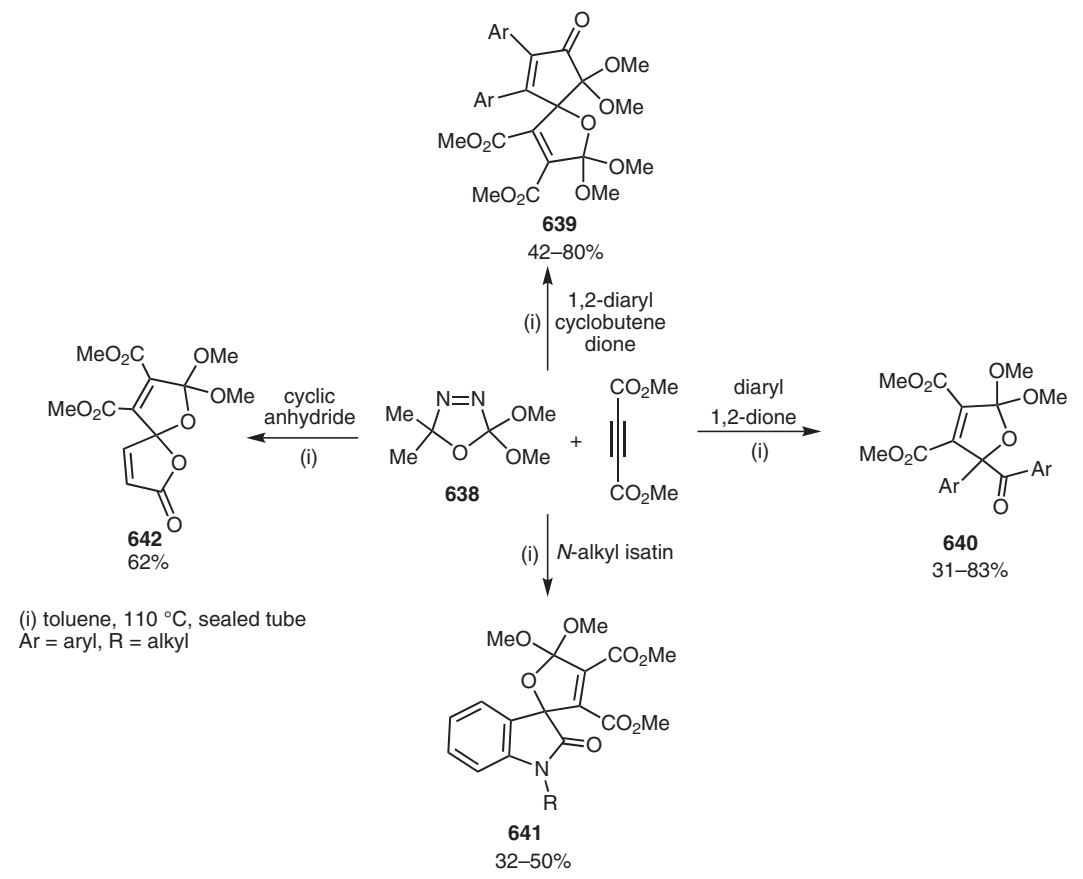

Scheme 158

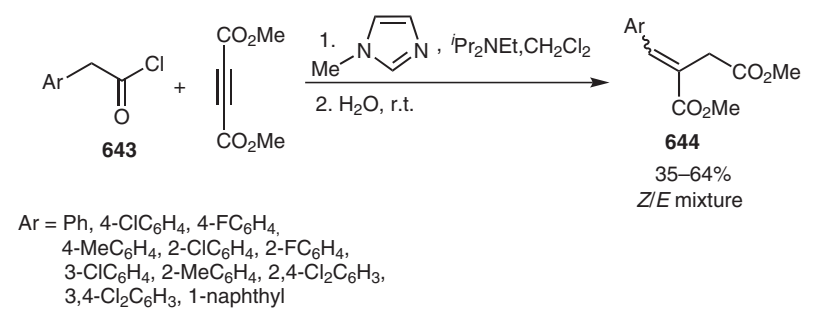

Scheme 159

\section{Conclusion}

In conclusion, we have presented here an overview of the recent progress in the chemistry of DMAD as a unique reagent with significant application in organic synthesis and medicinal chemistry. It also plays a pivotal role in multicomponent chemistry, participating in many diverse synthetic pathways. The high synthetic potential of this very accessible reagent has resulted in numerous applications, especially for the synthesis of complex heterocyclic structures. The increasing number of citations clearly shows the great importance of this simple but powerful reagent, and it is believed that additional new and useful DMAD chemistry will be discovered in the future.

\section{References}

(1) Sahoo, M. K. Synlett 2007, 2142.

(2) Diels, O.; Alder, K.; Nienburg, H.; Schmalbeck, O. Ann. Chem. 1931, 490, 243.

(3) Acheson, R. M.; Elmore, N. F. Adv. Heterocycl. Chem. 1978, 23, 263.

(4) Vedejs, E.; Grissom, J. W. J. Org. Chem. 1988, 53, 1882.
(5) Fodor, L.; Csomós, P.; Bernáth, G.; Sohár, P.; Holczbauer, T.; Kálmán, A. Monatsh. Chem. 2010, 141, 431.

(6) Bandrowski, E. Ber. Dtsch. Chem. Ges. 1877, 10, 838.

(7) Imrich, J.; Tomaščiková, J.; Danihel, I.; Kristian, P.; Böhm, S.; Klika, K. D. Heterocycles 2010, 80, 489.

(8) (a) Böhm, S.; Tomaščiková, J.; Imrich, J.; Danihel, I.; Kristian, P.; Koch, A.; Kleinpeter, E.; Klika, K. D. J. Mol. Struct. 2009, 916, 105. (b) Tomaščiková, J.; Imrich, J.; Danihel, I.; Böhm, S.; Kristian, P.; Pisarčiková, J.; Sabol, M.; Klika, K. D. Molecules 2008, 13, 501.

(9) Ahmadi, A.; Saidi, K.; Khabazzadeh, H. Mol. Divers. 2009, 13,353 .

(10) Darehkordi, A.; Saidi, K.; Islami, M. R. ARKIVOC 2007, (i), 180.

(11) Tomaščiková, J.; Danihel, I.; Böhm, S.; Imrich, J.; Kristian, P.; Potočňák, I.; Čejka, J.; Klika, K. D. J. Mol. Struct. 2008, $875,419$.

(12) Aly, A. A.; El-Shaieb, K. M. J. Chem. Res. 2007, 563.

(13) Ferrocenes; Togni, A.; Hayashi, T., Eds.; Wiley-VCH: Weinheim, 1995.

(14) Balázs, F.; Csámpai, A.; Nagy, T. Z.; Czugler, M.; Sohár, P. J. Organomet. Chem. 2009, 694, 3732.

(15) Fábián, B.; Kudar, V.; Csámpai, A.; Nagy, T. Z.; Sohár, P. J. Organomet. Chem. 2007, 692, 5621.

(16) Nami, N.; Gholami, F.; Vahedi, H.; Nami, N. Phosphorus, Sulfur Silicon Relat. Elem. 2007, 182, 2157.

(17) Muelas, S.; Di Maio, R.; Cerecetto, H.; Seoane, G.; Ochoa, C.; Escario, J. A.; Gómez-Barrio, A. Folia Parasitol. 2001, $48,105$.

(18) Neochoritis, C.; Eleftheriadis, N.; Tsoleridis, C. A.; Stephanidou-Stephanatou, J. Tetrahedron 2010, 66, 709.

(19) Bakulev, V. A.; Berseneva, V. S.; Belskaia, N. P.; Morzherin, Y. Y.; Zaitsev, A.; Dehaen, W.; Luyten, I.; Toppet, S. Org. Biomol. Chem. 2003, 1, 134.

(20) Ziyaei-Halimehjani, A.; Saidi, M. R. Tetrahedron Lett. 2008, 49, 1244.

(21) Hergué, N.; Mallet, C.; Touvron, J.; Allain, M.; Leriche, P.; Frère, P. Tetrahedron Lett. 2008, 49, 2425. 
(22) Yavari, I.; Sirouspour, M.; Souri, S. Helv. Chim. Acta 2010, 93, 2176.

(23) Fodor, L.; Csomós, P.; Csámpai, A.; Sohár, P.; Holczbauer, T.; Kálmán, A. Tetrahedron Lett. 2011, 52, 592.

(24) Voskressensky, L. G.; Borisova, T. N.; Kulikova, L. N.; Varlamov, A. V. Chem. Heterocycl. Compd. 2007, 43, 913

(25) Varlamov, A. V.; Borisova, T. N.; Voskressensky, L. G.; Soklakova, T. A.; Kulikova, L. N.; Chernyshev, A. I.; Alexandrov, G. G. Tetrahedron Lett. 2002, 43, 6767.

(26) Borisova, T. N.; Voskressensky, L. G.; Soklakova, T. A.; Kulikova, L. N.; Varlamov, A. V. Mol. Divers. 2003, 6, 207.

(27) Voskressensky, L. G.; Borisova, T. N.; Vorob'ev, I. V.; Postika, N. M.; Sorokina, E. A.; Varlamov, A. V. Russ. Chem. Bull. 2008, 57, 1547.

(28) Voskressensky, L. G.; Akbulatov, S. V.; Borisova, T. N.; Varlamov, A. V. Tetrahedron 2006, 62, 12392.

(29) Voskressensky, L. G.; Borisova, T. N.; Ovcharov, M. V.; Kulikova, L. N.; Sorokina, E. A.; Borisov, R. S.; Varlamov, A. V. Chem. Heterocycl. Compd. 2008, 44, 1510.

(30) De Silva, H. I.; Henry, W. P.; Pittman, C. U. Synthesis 2012, 44, 3337.

(31) Lenev, D. A.; Chervin, I. I.; Lyssenko, K. A.; Kostyanovsky, R. G. Tetrahedron Lett. 2007, 48, 3363.

(32) Voskressensky, L. G.; Borisova, T. N.; Kulikova, L. N.; Varlamov, A. V.; Catto, M.; Altomare, C.; Carotti, A. Eur. J. Org. Chem. 2004, 3128.

(33) González-Gómez, A.; Domínguez, G.; Amador, U.; PérezCastells, J. Tetrahedron Lett. 2008, 49, 5467.

(34) Zewge, D.; Chen, C. Y.; Deer, C.; Dormer, P. G.; Hughes, D. L. J. Org. Chem. 2007, 72, 4276.

(35) Nami, N.; Neumuller, B.; Heravi, M. M.; Haghdadi, M. Mendeleev Commun. 2008, 18, 153.

(36) Abdel-Megid, M. Chem. Heterocycl. Compd. (Engl. Transl.) 2009, 45, 1523 .

(37) Ramesh, K.; Murthy, S. N.; Karnakar, K.; Nageswar, Y. V. D. Tetrahedron Lett. 2011, 52, 3937.

(38) Anary-Abbasinejad, M.; Charkhati, K.; Anaraki-Ardakani, H. Synlett 2009, 1115.

(39) Ramesh, S.; Nagarajan, R. Synthesis 2011, 20, 3307.

(40) Vijesh, A. M.; Isloor, A. M.; Telkar, S.; Peethambar, S. K.; Sankappa, R.; Isloor, N. Eur. J. Med. Chem. 2011, 46, 3531.

(41) Heravi, M. M.; Nami, N.; Oskooie, H. A.; Hekmatshoar, R. Phosphorus, Sulfur Silicon Relat. Elem. 2006, 181, 87.

(42) Shestakov, A. S.; Sidorenko, O. E.; Shikhaliev, K. S. Russ. Chem. Bull. 2007, 56, 1423.

(43) Zaleska, B.; Karelus, M.; Serda, P. Synthesis 2010, 1654.

(44) Humphrey, G. R.; Pye, P. J.; Zhong, Y. L.; Angelaud, R.; Askin, D.; Belyk, K. M.; Maligres, P. E.; Mancheno, D. E.; Miller, R. A.; Reamer, R. A.; Weissman, S. A. Org. Process Res. Dev. 2011, 15, 73 .

(45) Ngwerume, S.; Lewis, W.; Camp, J. E. J. Org. Chem. 2013, 78,920 .

(46) (a) Haase, M.; Günther, W.; Görls, H.; Anders, E. Synthesis 1999, 2071. (b) Moonen, K.; Laureyn, I.; Stevens, C. V. Chem. Rev. 2004, 104, 6177.

(47) (a) Arango, E. P.; Iglesias, M. J.; Álvarez-Manzaneda, R.; Ortiz, F. L. ARKIVOC 2007, (iv), 102. (b) Ortiz, F. L.; Arango, E. P. J. Chem. Soc., Perkin Trans. 1 1996, 1481.

(48) Jiang, X. P.; Cheng, Y.; Shi, G. F.; Kang, Z. M. J. Org. Chem. 2007, 72, 2212.

(49) Li, X.; Wang, J. Y.; Yu, W.; Wu, L. M. Tetrahedron 2009, $65,1140$.

(50) Gaywood, A. P.; McNab, H. J. Org. Chem. 2009, 74, 4278.

(51) (a) Osborne, C. S.; Leitner, I.; Hofbauer, B.; Fielding, C. A.; Favre, B.; Ryder, N. S. Antimicrob. Agents Chemother. 2006, 50, 2234. (b) Takano, R.; Sugano, K.; Higashida, A.;
Hayashi, Y.; Machida, M.; Aso, Y.; Yamashita, S. Pharm. Res. 2006, 23, 1144. (c) Xue, C.; Li, T.; Deng, Z.; Fu, H.; Lin, W. Pharmazie 2006, 61, 1041. (d) Phelps, J. B.; Hoffman, W. P.; Lee, C.; Murphy, G. P.; Garriott, M. L. Mutat. Res. 2004, 561, 153. (e) Rosefort, C.; Fauth, E.; Zankl, H. Mutagenesis 2004, 19, 277. (f) Kinobe, R. T.; Dercho, R. A.; Vlahakis, J. Z.; Brien, J. F.; Szarek, W. A.; Nakatsu, K. J. Pharmacol. Exp. Ther. 2006, 319, 277.

(52) Abid, O. U. R.; Ibad, M. F.; Nawaz, M.; Adeel, M.; Rama, N. H.; Villinger, A.; Langer, P. Tetrahedron Lett. 2010, 51, 657.

(53) Kotha, S.; Halder, S.; Brahmachary, E. Tetrahedron 2002, 58,9203 .

(54) Takekuma, S. I.; Matsuoka, H.; Minematsu, T.; Takekuma, H. Tetrahedron 2010, 66, 3004.

(55) Hopf, H.; Yildizhan, S. Eur. J. Org. Chem. 2011, 2029.

(56) Ogasawara, M.; Watanabe, S.; Nakajima, K.; Takahashi, T. Organometallics 2008, 27, 6565 .

(57) Nair, V.; Vellalath, S.; Babu, B. P.; Vargehese, V.; Paul, R. R.; Suresh, E. Org. Biomol. Chem. 2010, 8, 4861.

(58) Nair, V.; Menon, R. S.; Biju, A. T.; Sinu, C. R.; Paul, R. R.; Jose, A.; Sreekumar, V. Chem. Soc. Rev. 2011, 40, 5336.

(59) Fan, W. Y.; Wang, Z. L.; Zhang, Z. G.; Li, H. C.; Deng, W. P. Tetrahedron 2011, 67, 5596 .

(60) Sher, M.; Fischer, C.; Reinke, H.; Langer, P. Tetrahedron 2007, 63, 4080.

(61) Okamura, H.; Iiji, H.; Hamada, T.; Iwagawa, T.; Furuno, H. Tetrahedron 2009, 65, 10709.

(62) Halton, B. Chem. Rev. 2003, 103, 1327.

(63) Cox, J. R.; Simpson, J. H.; Swager, T. M. J. Am. Chem. Soc. 2013, 135, 640.

(64) (a) Pavan, M. P.; Chakravarty, M.; Swamy, K. C. K. Eur. J. Org. Chem. 2009, 5927. (b) Kumar, N. N. B.; Reddy, M. N.; Swamy, K. C. K. J. Org. Chem. 2009, 74, 5395. (c) Balaraman, E.; Srinivas, V.; Swamy, K. C. K. Tetrahedron 2009, 65, 7603.

(65) Kalek, M.; Johansson, T.; Jezowska, M.; Stawinski, J. Org. Lett. 2010, 12, 4702.

(66) Sajna, K. V.; Kotikalapudi, R.; Chakravarty, M.; Kumar, N. N. B.; Swamy, K. C. K. J. Org. Chem. 2011, 76, 920

(67) Feng, C.; Wang, X.; Wang, B. Q.; Zhao, K. Q.; Hu, P.; Shi, Z. J. Chem. Commun. 2012, 48, 356.

(68) Neochoritis, C.; Livadiotou, D.; Stephanidou-Stephanatou, J.; Tsoleridis, C. A. Tetrahedron Lett. 2007, 48, 2275.

(69) Segura, J. L.; Martín, N. Chem. Rev. 1999, 99, 3199.

(70) Sato, R.; Chino, K.; Saito, M. Sulfur Lett. 1990, 10, 233.

(71) Konstantinova, L. S.; Rakitin, O. A.; Rees, C. W. Chem. Rev. 2004, 104, 2617.

(72) (a) Kuninobu, Y.; Kawata, A.; Nishi, M.; Takata, H.; Takai, K. Chem. Commun. 2008, 6360. (b) Yao, T.; Larock, R. C. J. Org. Chem. 2003, 68, 5936. (c) Park, K. H.; Jung, I. G.; Chung, Y. K. Synlett 2004, 2541. (d) Oh, D. C.; Gontang, E. A.; Kauffman, C. A.; Jensen, P. R.; Fenical, W. J. Nat. Prod. 2008, 71, 570. (e) Trisuwan, K.; Rukachaisirikul, V.; Sukpondma, Y.; Preedanon, S.; Phongpaichit, S.; Rungjindamai, N.; Sakayaroj, J. J. Nat. Prod. 2008, 71, 1323. (f) Kuninobu, Y.; Takata, H.; Kawata, A.; Takai, K. Org. Lett. 2008, 10, 3133.

(73) Kim, E. S.; Kim, K. H.; Kim, S. H.; Kim, J. N. Tetrahedron Lett. 2009, 50, 5098.

(74) Narayan, A. R. H.; Sarpong, R. Org. Biomol. Chem. 2012, 10,70 .

(75) Ram, R. N.; Kumar, N. Tetrahedron Lett. 2008, 49, 799.

(76) Ram, R. N.; Kumar, N. Tetrahedron 2008, 64, 10267.

(77) Shinohara, H.; Sonoda, M.; Atobe, S.; Masuno, H.; Ogawa, A. Tetrahedron Lett. 2011, 52, 6238. 
(78) Zubkov, F. I.; Zaitsev, V. P.; Orlova, A. A.; Peregudov, A. S.; Mikhailova, N. M.; Varlamov, A. V. Russ. J. Org. Chem. 2007, 43, 1202.

(79) Chen, Z.; Trudell, M. L. Chem. Rev. 1996, 96, 1179.

(80) (a) Sha, C. K.; Liu, J. M.; Chiang, R. K.; Wang, S. L. Heterocycles 1990, 31, 603. (b) Sha, C. K.; Yang, J. F.; Chang, C. J. Tetrahedron Lett. 1996, 37, 3487.

(81) Ikeuchi, Y.; Saitoh, T.; Taguchi, T.; Hanzawa, Y. Tetrahedron Lett. 2007, 48, 2477.

(82) (a) Diver, S. T.; Giessert, A. J. Chem. Rev. 2004, 104, 1317. (b) Blechert, S.; Connon, S. J. Angew. Chem. Int. Ed. 2003, 42, 1900. (c) Hansen, E. C.; Lee, D. Org. Lett. 2004, 6, 2035. (d) Brenneman, J. B.; Martin, S. F. Org. Lett. 2004, 6, 1329

(83) (a) Trofimov, B. A.; Sobenina, L. N.; Demenev, A. P.; Mikhaleva, A. I. Chem. Rev. 2004, 104, 2481. (b) Ohno, M.; Shimizu, S.; Eguchi, S. Heterocycles 1991, 32, 1199. (c) Yoshida, T.; Ito, A.; Ibusuki, K.; Murase, M.; Kotani, E. Chem. Pharm. Bull. 2001, 49, 1198. (d) Joseph, B.; Da Costa, H.; Merour, J.-Y.; Leonce, S. Tetrahedron 2000, 56, 3189.

(84) Singh, P.; Bisetty, K.; Mahajan, M. P. S. Afr. J. Chem. 2009, $62,47$.

(85) Singh, P.; Sharma, P.; Bisetty, K.; Mahajan, M. P. Tetrahedron 2009, 65, 8478.

(86) Al-Awadi, N. A.; Ibrahim, M. R.; Abdelhamid, I. A.; Elnagdi, M. H. Tetrahedron 2008, 64, 8202.

(87) (a) Kopka, I. E. Tetrahedron Lett. 1988, 29, 3765. (b) Crank, G.; Khan, H. R. J. Heterocycl. Chem. 1985, 22, 1281.

(88) (a) Dondoni, A.; Medici, A.; Venturoli, C. J. Org. Chem. 1980, 45, 621. (b) Medici, A.; Pedrini, P.; Venturoli, C.; Dondoni, A. J. Org. Chem. 1981, 46, 2790.

(89) Husinec, S.; Savic, V. Tetrahedron: Asymmetry 2005, 16, 2047.

(90) Caira, M. R.; Georgescu, E.; Barbu, L.; Georgescu, F.; Dumitrascu, F. ARKIVOC 2011, $(x), 44$

(91) Shang, Y.; Zhang, M.; Yu, S.; Ju, K.; Wang, C.; He, X. Tetrahedron Lett. 2009, 50, 6981.

(92) Hazra, A.; Mondal, S.; Maity, A.; Naskar, S.; Saha, P.; Paira, R.; Sahu, K. B.; Paira, P.; Ghosh, S.; Sinha, C.; Samanta, A.; Banerjee, S.; Mondal, N. B. Eur. J. Med. Chem. 2011, 46, 2132.

(93) Krchňák, V.; Holladay, M. W. Chem. Rev. 2002, 102, 61.

(94) (a) Washizuka, K. I.; Nagai, K.; Minakata, S.; Ryu, I.; Komatsu, M. Tetrahedron Lett. 2000, 41, 691. (b) Washizuka, K. I.; Nagai, K.; Nagai, K.; Minakata, S.; Ryu, I.; Komatsu, M. Tetrahedron Lett. 1999, 40, 8849.

(95) Mlostoń, G.; Jasiński, M.; Heimgartner, H. Eur. J. Org. Chem. 2011, 2542.

(96) Chakraborty, B.; Luitel, G. P. Tetrahedron Lett. 2013, 54, 765 .

(97) (a) Huisgen, R. In 1,3-Dipolar Cycloaddition Chemistry; Vol. 1; Padwa, A., Ed.; Wiley: New York, 1984, 1-176. (b) Huisgen, R. Proc. Chem. Soc. 1961, 357. (c) Huisgen, R. Pure Appl. Chem. 1989, 61, 613.

(98) Nabiev, O. G.; Nabizade, Z. O.; Kostyanovsky, R. G. Mendeleev Commun. 2009, 19, 281.

(99) Taherpour, A. A.; Kheradmand, K. J. Heterocycl. Chem. 2009, 46, 131.

(100) Kumar, I.; Rode, C. V. Chem. Lett. 2007, 36, 592.

(101) Semakin, A. N.; Sukhorukov, A. Y.; Lesiv, A. V.; Khomutova, Y. A.; Ioffe, S. L. Russ. J. Org. Chem. 2007, 43, 1218.

(102) Ding, Q.; Wang, Z.; Wu, J. J. Org. Chem. 2009, 74, 921.

(103) Strakova, I.; Strakovs, A.; Petrova, M.; Belyakov, S. Chem. Heterocycl. Compd. (Engl. Transl.) 2007, 43, 1512.
(104) Barriga, S.; Fuertes, P.; Marcos, C. F.; Miguel, D.; Rakitin, O. A.; Rees, C. W.; Torroba, T. J. Org. Chem. 2001, 66, 5766.

(105) Konstantinova, L. S.; Lysov, K. A.; Amelichev, S. A.; Obruchnikova, N. V. Rakitin O. A. Tetrahedron 2009, 65, 2178.

(106) Rees, C. W.; Rakitin, O. A.; Marcos, C. F.; Torroba, T. J. Org. Chem. 1999, 64, 4376.

(107) Wudl, F.; Smith, G. M.; Hufnagel, E. J. J. Chem. Soc., Sect. D: Chem. Commun. 1970, 1453.

(108) Rice, J. E.; Okamoto, Y. J. Org. Chem. 1981, 46, 446.

(109) Benitez, F. M.; Grunwell, J. R. J. Org. Chem. 1978, 43, 2917.

(110) Sato, M.; Gonnella, N. C.; Cava, M. P. J. Org. Chem. 1979, 44, 930 .

(111) (a) Gorgues, A.; Hudhomme, P.; Sallé, M. Chem. Rev. 2004, 104, 5151. (b) Lakshmikantham, M. V.; Cava, M. P. J. Org. Chem. 1980, 45, 2632.

(112) Kuroda, S.; Kasai, R.; Nagaya, R.; Miyatake, R.; Horino, Y.; Matsumoto, N.; Oda, M. Tetrahedron Lett. 2011, 52, 1426.

(113) Uršič, U.; Grošelj, U.; Meden, A.; Svete, J.; Stanovnik, B. Tetrahedron Lett. 2008, 49, 3775.

(114) Uršič, U.; Grošelj, U.; Meden, A.; Svete, J.; Stanovnik, B. Helv. Chim. Acta 2009, 92, 481.

(115) Alajarín, M.; Cabrera, J.; Pastor, A.; Sánchez-Andrada, P.; Bautista, D. J. Org. Chem. 2006, 71, 5328.

(116) Chen, Y.; Ye, S.; Jiao, L.; Liang, Y.; Sinha-Mahapatra, D. K.; Herndon, J. W.; Yu, Z. X. J. Am. Chem. Soc. 2007, 129, 10773.

(117) Kuznetsov, A. G.; Bush, A. A.; Babaev, E. V. Tetrahedron 2008, 64, 749 .

(118) (a) Luo, Y.; Herndon, J. W.; Cervantes-Lee, F. J. Am. Chem. Soc. 2003, 125, 12720. (b) Zhang, L.; Wang, Y.; Buckingham, C.; Herndon, J. W. Org. Lett. 2005, 7, 1665.

(119) Roy, P.; Ghorai, B. K. Tetrahedron Lett. 2011, 52, 5668.

(120) Ramazani, A.; Kazemizadeh, A. R. Curr. Org. Chem. 2011, 15,3986

(121) Yavari, I.; Islami, M. R.; Habibi, A.; Tikdari, A. M.; Ebrahimi, L. Phosphorus, Sulfur Silicon Relat. Elem. 2004, 179,575 .

(122) Mosslemin, M. H.; Yavari, I.; Anary-Abbasinejad, M.; Nateghi, M. R. Synthesis 2004, 1029.

(123) Asghari, S.; Baharfar, R.; Safiri, S. Phosphorus, Sulfur Silicon Relat. Elem. 2005, 180, 2805.

(124) Yavari, I.; Mohtat, B.; Zare, H.; Alborzi, A. R. Mendeleev Commun. 2005, 15, 250.

(125) Yavari, I.; Adib, M.; Sayahi, M. H. J. Chem. Soc., Perkin Trans. 1 2002, 1517.

(126) Yavari, I.; Esmaili, A. A.; Ramazani, A.; Bolbol-Amiri, A. R. Monatsh. Chem. 1997, 128, 927.

(127) Yavari, I.; Adib, M.; Jahani-Moghaddam, F.; Bijanzadeh, H. R. Tetrahedron 2002, 58, 6901.

(128) Islami, M. R.; Mollazehi, F.; Hassani, Z. Russ. Chem. Bull. 2007, 56, 532 .

(129) Ahmadi, A.; Saidi, K.; Sheibani, H.; Khabazzadeh, H.; Molahoseini, A. Phosphorus, Sulfur Silicon Relat. Elem. 2007, 82, 1225.

(130) Johnson, A. W.; Tebby, J. C. J. Chem. Soc. 1961, 2126.

(131) Nair, V.; Rajeev, S.; Menon, A. R.; Sreekanth, N.; Abhilash, A.; Biju, T. Acc. Chem. Res. 2006, 39, 520.

(132) Bayat, M.; Imanieh, H.; Hassanzadeh, F. Tetrahedron Lett. 2010, 51, 1873.

(133) Anary-Abbasinejad, M.; Shams, N.; Hassanabadi, A. Phosphorus, Sulfur Silicon Relat. Elem. 2010, 185, 1823.

(134) Anary-Abbasinejad, M.; Mazraeh-Seffid, M.; Poorhassan, E.; Hassanabadi, A.; Rastegari, F. ARKIVOC 2008, (xvii), 265 . 
(135) (a) Yavari, I.; Hekmat-Shoar, R.; Zonouzi, A. Tetrahedron Lett. 1998, 39, 2391. (b) Yavari, I.; Adib, M.; Hojabri, L. Tetrahedron 2001, 57, 7537. (c) Yavari, I.; AnaryAbbasinejad, M.; Hossaini, Z. Org. Biomol. Chem. 2003, 1, 560 .

(136) Majumdar, K. C.; Ansary, I.; Samanta, S.; Brindaban, R. Synlett 2011, 694

(137) Symeonidis, T.; Fylaktakidou, K. C.; Hadjipavlou-Litina, D. J.; Litinas, K. E. Eur. J. Med. Chem. 2009, 44, 5012.

(138) Yavari, I.; Alizadeh, A. A.; Anary-Abbasinejad, M. Phosphorus, Sulfur Silicon Relat. Elem. 2003, 178, 269.

(139) Islami, M. R.; Hassani, Z.; Saidi, K. Synth. Commun. 2003, 33,65 .

(140) (a) Esmaili, A. A.; Ghereghloo, M.; Islami, M. R.; Bijanzadeh, H. R. Tetrahedron 2003, 59, 4785.

(b) Saghatforoush, L.; Maghsoodlou, M. T.; Aminkhani, A.; Marandi, G.; Kabiri, R. J. Sulfur Chem. 2006, 27, 583.

(141) Khabazzadeha, H.; Saidia, K.; Sheibania, H.; Islamia, M. R. Phosphorus, Sulfur Silicon Relat. Elem. 2007, 182, 2163.

(142) (a) Waldmann, H.; Khedkar, V.; Dückert, H.; Schürmann, M.; Oppel, I. M.; Kumar, K. Angew. Chem. Int. Ed. 2008, 47, 6869. (b) Dückert, H.; Khedkar, V.; Waldmann, H.; Kumar, K. Chem. Eur. J. 2011, 17, 5130. (c) Dückert, H.; Pries, V.; Khedkar, V.; Menninger, S.; Bruss, H.; Bird, A. W.; Maliga, Z.; Brockmeyer, A.; Janning, P.; Hyman, A.; Grimme, S.; Schürmann, M.; Preut, H.; Hübel, K.; Ziegler, S.; Kumar, K.; Waldmann, H. Nat. Chem. Biol. 2012, 8, 179.

(d) Eschenbrenner-Lux, V.; Dückert, H.; Khedkar, V.; Bruss, H.; Waldmann, H.; Kumar, K. Chem. Eur. J. 2013 , 19, 2294.

(143) Maghsoodlou, M. T.; Hazeri, N.; Habibi-Khorassani, S. M.; Heydari, R.; Marandi, G.; Lashkari, M.; Bagherpour, K.; Gharechaei, Z. Monatsh. Chem. 2010, 141, 351.

(144) (a) Maghsoodlou, M. T.; Charati, F. R.; Khorassani, S. M. H.; Ghasemzadeh, M.; Makha, M. J. Chem. Res. 2008, 55. (b) Ziyaadini, M.; Maghsoodlou, M. T.; Hazeri, N.; HabibiKhorassani, S. M. Heteroat. Chem. 2013, 24, 84.

(145) Adib, M.; Sheikhi, E.; Moghaddam, G. S.; Bijanzadeh, H. R. Tetrahedron Lett. 2010, 51, 5646.

(146) (a) Anary-Abbasinejad, M.; Hassanabadi, A. J. Chem. Res. 2007, 475. (b) Anary-Abbasinejad, M.; Hassanabadi, A.; Mazraeh-Seffid, M. J. Chem. Res. 2007, 708

(147) Deng, J.-C.; Chan, F.-W.; Kuo, C.-W.; Cheng, C.-A.; Huang, C.-Y.; Chuang, S.-C. Eur. J. Org. Chem. 2012, 5738

(148) Nishimura, Y.; Kawamura, Y.; Watanabe, Y.; Hayashi, M. J. Org. Chem. 2010, 75, 3875.

(149) (a) Hanawalt, E. M.; Doxsee, K. M.; Shen, G. S.; Weakley, T. J. R.; Knobler, C. B.; Hope, H. Heteroat. Chem. 1998, 9 , 9. (b) Holah, D. G.; Hughes, A. N.; Kleemola, D.

J. Heterocycl. Chem. 1978, 15, 1319. (c) Duan, Z.; Zhang, J.; Tian, R.; Bai, L.; Mathey, F. Organometallics 2008, 27, 5169.

(150) Sun, J.; Xia, E. Y.; Wu, Q.; Yan, C. G. ACS Comb. Sci. 2011, $13,421$.

(151) (a) Sun, J.; Xia, E. Y.; Wu, Q.; Yan, C. G. Org. Lett. 2010, 12, 3678. (b) Khan, A. T.; Ghosh, A.; Khan, Md. M. Tetrahedron Lett. 2012, 53, 2622.

(152) (a) Khan, A. T.; Khan, Md. M. Tetrahedron Lett. 2011, 52 , 3455. (b) Balalaie, S.; Baoosi, L.; Tahoori, F.; Rominge, F.; Bijanzadeh, H. R. Tetrahedron 2013, 69, 738.

(153) (a) Das, B.; Reddy, G. C.; Balasubramanyam, P.; Veeranjaneyulu, B. Synthesis 2010, 1625. (b) Nagarapu, L.; Mallepalli, R.; Yeramanchi, L.; Bantu, R. Tetrahedron Lett. 2011, 52, 3401

(154) Hadjebi, M.; Hashtroudi, M. S.; Bijanzadeh, H. R.; Balalaie, S. Helv. Chim. Acta 2011, 94, 382.

(155) Nasiri, F.; Atashkar, B. Monatsh. Chem. 2008, 139, 1223.
(156) Terzidis, M. A.; Stephanidou-Stephanatou, J.; Tsoleridis, C. A. Tetrahedron Lett. 2009, 50, 1196.

(157) (a) Diels, O.; Alder, K. Justus Liebigs Ann. Chem. 1932 498, 16. (b) Huisgen, R. Chem. Ber. 1967, 1094. (c) Nair, V.; Sreekanth, A. R.; Vinod, A. U. Org. Lett. 2001, 3, 3495.

(158) Nair, V.; Pillai, A. N.; Menon, R. S.; Suresh, E. Org. Lett. 2005, 7, 1189 .

(159) Khalilzadeh, M. A.; Hossaini, Z.; Kharian, S. Mol. Divers. 2009, 14, 605 .

(160) Maghsoodlou, M. T.; Habibi-Khorassani, S. M.; Moradi, A.; Hazeri, N.; Davodi, A.; Sajadikhah, S. S. Tetrahedron 2011, 67, 8492

(161) Asghari, S.; Habibi, A. K. Tetrahedron 2012, 68, 8890.

(162) Nair, V.; Pillai, A. N.; Beneesh, P. B.; Suresh, E. Org. Lett. 2005, 7, 4625 .

(163) (a) Terzidis, M. A.; Tsiaras, V. G.; StephanidouStephanatou, J.; Tsoleridis, C. A. Synthesis 2011, 97. (b) Terzidis, M. A.; Dimitriadou, E.; Tsoleridis, C. A.; Stephanidou-Stephanatou, J. Tetrahedron Lett. 2009, 50, 2174 .

(164) (a) Nair, V.; Devipriya, S.; Suresh, E. Tetrahedron 2008, 64, 3567. (b) Diels, O. Justus Liebigs Ann. Chem. 1933, 505, 103. (c) Valizadeh, H.; Shomali, A.; Gholipour, H. J. Heterocycl. Chem. 2011, 48, 1440.

(165) Huisgen, R.; Morikawa, M.; Herbig, K.; Brunn, E. Chem. Ber. 1967, 100, 1094.

(166) Nair, V.; Devi, B. R.; Varma, L. R. Tetrahedron Lett. 2005, 46,5333

(167) Nair, V.; Sreekanth, A. R.; Abhilash, N.; Bhadbhade, M. M.; Gonnade, R. C. Org. Lett. 2002, 4, 3575.

(168) Nair, V.; Sreekanth, A. R.; Biju, A. T.; Rath, N. P. Tetrahedron Lett. 2003, 44, 729.

(169) Yavari, I.; Piltan, M.; Moradi, L. Tetrahedron 2009, 65, 2067.

(170) Ruijter, E.; Scheffelaar, R.; Orru, R. V. A. Angew. Chem. Int. Ed. 2011, 50, 6234 .

(171) Nassiri, M.; Maghsoodlou, M. T.; Heydari, R.; Mostafa, S.; Khorassani, H. Mol. Divers. 2008, 12, 111.

(172) Yadav, J. S.; Reddy, B. V. S.; Yadav, N. N.; Gupta, M. K. Tetrahedron Lett. 2008, 49, 2815.

(173) Yavari, I.; Ghazanfarpour-Darjani, M.; Sabbaghan, M.; Hossaini, Z. Tetrahedron Lett. 2007, 48, 3749.

(174) Li, M.; Pan, L.; Wen, L. R. Helv. Chim. Acta 2011, 94, 169.

(175) Terzidis, M. A.; Tsoleridis, C. A.; StephanidouStephanatou, J. Synlett 2009, 229.

(176) Safaei, S.; Mohammadpoor-Baltork, I.; Khosropour, A. R.; Moghadam, M.; Tangestaninejad, S.; Mirkhani, V. $A d v$. Synth. Catal. 2012, 354, 3095.

(177) (a) Winterfeldt, E.; Schumann, D.; Dillinger, H.-J. Chem. Ber. 1969, 102, 1656. (b) Dömling, A. Chem. Rev. 2006, 106, 17. (c) Dömling, A.; Wang, W.; Wang, K. Chem. Rev. 2012, 112, 3083 .

(178) Yavari, I.; Mokhtarporyani-Sanandaj, A.; Moradi, L.; Mirzaei, A. Tetrahedron 2008, 64, 5221.

(179) Alizadeh, A.; Rostamnia, S.; Zhu, L. G. Tetrahedron Lett. 2010, 51,4750 .

(180) Nair, V.; Dhanya, R.; Viji, S. Tetrahedron 2005, 61, 5843.

(181) Yadav, J. S.; Reddy, B. V. S.; Shubashree, S.; Sadashiv, K.; Naidu, J. J. Synthesis 2004, 2376.

(182) Yadav, J. S.; Reddy, B. V. S.; Shubashree, S.; Sadashiv, K.; Rao, D. K. J. Mol. Catal. A: Chem. 2007, 272, 128.

(183) (a) Nair, V.; Vinod, A. U. Chem. Commun. 2000, 1019. (b) Reddy, B. V. S.; Reddy, A. M.; Somashekar, D.; Yadav, J. S.; Sridhar, B. Synthesis 2010, 2571. (c) Ghandi, M.; Zarezadeh, N. Tetrahedron 2013, 69, 8668.

(184) Terzidis, M. A.; Stephanidou-Stephanatou, J.; Tsoleridis, C. A. J. Org. Chem. 2010, 75, 1948 . 
(185) Hazeri, N.; Maghsoodlou, M. T.; Habibi-Khorassani, S. M.; Marandi, G.; Khandan-Barani, K.; Ziyaadini, M.; Aminkhani, A. ARKIVOC 2007, (i), 173.

(186) Nair, V.; Deepthi, A. Tetrahedron Lett. 2006, 47, 2037.

(187) Shaabani, A.; Rezayan, A. H.; Ghasemi, S.; Sarvary, A. Tetrahedron Lett. 2009, 50, 1456.

(188) Asghari, S.; Mohammadi, L. Tetrahedron Lett. 2006, 47, 4297.

(189) Hazeri, N.; Maghsoodlou, M. T.; Habibi-Khorassani, S. M.; Ziyaadini, M.; Marandi, G.; Khandan-Barani, K.; Bijanzadeh, H. R. ARKIVOC 2007, (xiii), 34.

(190) Nair, V.; Vinod, A. U.; Abhilash, N.; Menon, R. S.; Santhi, V.; Varma, R. L.; Viji, S.; Mathew, S. C.; Srinivas, R. Tetrahedron 2003, 59, 10279.

(191) (a) Nair, V.; Vinod, A. U.; Nair, J. S.; Sreekanth, A. R.; Rath, N. P. Tetrahedron Lett. 2000, 41, 6675. (b) Nair, V.; Nair, J. S.; Vinod, A. U. Synthesis 2000, 1713.

(192) Magdziak, D.; Meek, S. J.; Pettus, T. R. R. Chem. Rev. 2004, 104, 1383

(193) Yavari, I.; Sabbaghan, M.; Hossaini, Z. Monatsh. Chem. 2008, 625.

(194) Shaabani, A.; Soleimani, E.; Sarvary, A. Monatsh. Chem. 2008, 139, 629.

(195) Ramazani, A.; Holagh, M. V. Phosphorus, Sulfur Silicon Relat. Elem. 2009, 184, 171.

(196) Yavari, I.; Sanaeishoar, T.; Azad, L. Mendeleev Commun. 2011, 21, 229.

(197) Teimouri, M. B.; Shaabani, A.; Bazhrang, R. Tetrahedron 2006, 62, 1845.

(198) Shaabani, A.; Soleimani, E.; Khavasi, H. R.; Hoffmann, R. D.; Rodewald, U. C.; Pöttgen, R. Tetrahedron Lett. 2006, 47, 5493.

(199) Li, M.; Kong, W.; Wen, L.-R.; Liu, F.-H. Tetrahedron 2012, $68,4838$.

(200) Yavari, I.; Sirouspour, M.; Souri, S. Mol. Divers. 2006, 10, 265.

(201) Azizian, J.; Ramazani, A.; Haji, M. Helv. Chim. Acta 2011, 94, 371 .

(202) Teimouri, M. B.; Bazhrang, R.; Eslamimanesh, V.; Nouri, A. Tetrahedron 2006, 62, 3016.

(203) Shaabani, A.; Soleimani, E.; Sarvary, A.; Rezayan, A. H. Bioorg. Med. Chem. Lett. 2008, 18, 3968.

(204) Teimouri, M. B.; Bazhrang, R. Monatsh. Chem. 2009, 140, 513.
(205) Kabiri, R.; Hazeri, N.; Khorassani, S. M. H.; Maghsoodlou, M. T.; Ebrahimi, A.; Saghatforoush, L.; Marandi, G.; Razmjoo, Z. ARKIVOC 2008, (xvii), 12.

(206) Shaabani, A.; Sarvary, A.; Rezayan, A. H.; Keshipour, S. Tetrahedron 2009, 65, 3492.

(207) Akbarzadeh, R.; Amanpour, T.; Mirzaei, P.; Bazgir, A. Helv. Chim. Acta 2011, 94, 1527.

(208) Yavari, I.; Karimi, E.; Djahaniani, H. Synth. Commun. 2007, 2593.

(209) Adib, M.; Sayahi, M. H.; Ziyadi, H.; Bijanzadeh, H. R.; Zhu, L. G. Tetrahedron 2007, 63, 11135.

(210) Adib, M.; Sayahi, M. H.; Nosrati, M.; Zhu, L. G. Tetrahedron Lett. 2007, 48, 4195.

(211) Teimouri, M. B. Tetrahedron 2006, 62, 10849.

(212) Breslow, R. J. Am. Chem. Soc. 1958, 80, 3719.

(213) Wanzlick, H. W. Angew. Chem. Int. Ed. 1962, 1, 75.

(214) Arduengo, A. J.; Harlow, R. L.; Kline, M. J. Am. Chem. Soc. 1991, 113, 361.

(215) Herrmann, W. A. Angew. Chem. Int. Ed. 2002, 41, 1290.

(216) Movassaghi, M.; Schmidt, M. A. Org. Lett. 2005, 7, 2453.

(217) (a) Enders, D.; Balensiefer, T. Acc. Chem. Res. 2004, 37, 534. (b) Johnson, J. S. Angew. Chem. Int. Ed. 2004, 43, 1326.

(218) Ma, C.; Ding, H.; Wu, G.; Yang, Y. J. Org. Chem. 2005, 70, 8919.

(219) Ma, C.; Ding, H.; Zhang, Y.; Bian, M. Angew. Chem. Int. Ed. 2006, 45, 7793 .

(220) Nair, V.; Sreekumar, V.; Bindu, S.; Suresh, E. Org. Lett. 2005, 7, 12: 2297.

(221) Cheng, Y.; Peng, J. H.; Li, J. Q. J. Org. Chem. 2010, 75, 2382.

(222) Pan, H. R.; Wang, X. R.; Yan, C. X.; Sun, Z. X.; Cheng, Y. Org. Biomol. Chem. 2011, 9, 2166.

(223) Chaniyara, R.; Tala, S.; Chen, C.-W.; Lee, P.-C.; Kakadiya, R.; Dong, H.; Marvania, B.; Chen, C.-H.; Chou, T.-C.; Lee, T.-C.; Shah, A.; Su, T.-L. Eur. J. Med. Chem. 2012, 53, 28.

(224) Hamadi, H.; Khoobi, M.; Hossaini, Z.; Shafiee, A. Mol. Divers. 2011, 15, 35 .

(225) Nair, V.; Deepthi, A.; Poonoth, M.; Santhamma, B.; Vellalath, S.; Babu, B. P.; Mohan, R.; Suresh, E. J. Org. Chem. 2006, 71, 2313.

(226) Ding, F.; Ma, C.; Yang, Y.; Wang, Y. Org. Lett. 2005, 7, 2125 . 